\title{
South European spiders from the Duffey collection in the Manchester Museum (Arachnida: Araneae)
}

DOI:

10.13156/arac.2020.18.4.333

\section{Document Version}

Accepted author manuscript

Link to publication record in Manchester Research Explorer

\section{Citation for published version (APA):}

Breitling, R. (2020). South European spiders from the Duffey collection in the Manchester Museum (Arachnida: Araneae). Arachnology. https://doi.org/10.13156/arac.2020.18.4.333

\section{Published in:}

Arachnology

\section{Citing this paper}

Please note that where the full-text provided on Manchester Research Explorer is the Author Accepted Manuscript or Proof version this may differ from the final Published version. If citing, it is advised that you check and use the publisher's definitive version.

\section{General rights}

Copyright and moral rights for the publications made accessible in the Research Explorer are retained by the authors and/or other copyright owners and it is a condition of accessing publications that users recognise and abide by the legal requirements associated with these rights.

\section{Takedown policy}

If you believe that this document breaches copyright please refer to the University of Manchester's Takedown Procedures [http://man.ac.uk/04Y6Bo] or contact uml.scholarlycommunications@manchester.ac.uk providing relevant details, so we can investigate your claim.

\section{OPEN ACCESS}


1 South European spiders from the Duffey collection in the Manchester Museum

2 (Arachnida: Araneae)

3

4

5

6

7

8

Rainer Breitling

Faculty of Science and Engineering, University of Manchester, M1 7DN Manchester, UK; rainer.breitling@manchester.ac.uk

Short title: South European spiders

\begin{abstract}
Eric Duffey's spider collection in the Manchester Museum, accumulated over more than 40 years, contains more than 300 samples from a diverse range of biotopes in most South European countries. Most of this material was previously unsorted. It has now been sorted and identified to species level. It contains more than 2500 specimens of more than 500 species in 240 genera and 42 families. The collection details of all of these samples are listed here, and a number of selected specimens are illustrated.
\end{abstract}

\title{
Introduction
}

The spider collection donated by Eric Duffey to the Manchester Museum (Logunov 2011, Breitling 2018) contained 9 jars and one box of mostly undetermined and unsorted spiders predominantly from southern Europe. The earliest sample, from Tour du Valat in the Camargue (France), is dated 21 April 1956, and Duffey's collecting activities continued until at least 15 May 2003, when he was beating spiders from the south boundary hedge of his estate Chez Gouillard in Haute-Vienne (France). In between, samples were obtained (mostly by hand collecting, but also by pitfall traps, leaf litter extraction and other methods), from a wide range of habitats from Portugal and Spain (mostly the area around Huesca in the central Pyrenees) to north-east Greece and Cyprus. All this material, from more than 120 distinct locations in 12 countries, has now been sorted and has yielded about 1300 records based on more than 2500 specimens of more than 500 species in 240 genera and 42 families. 


\section{Material and Methods}

Specimens were sorted under a Cambridge Instruments Z45E stereo microscope (magnification up to $45 \times$ ). The methods for examining the material changed during the course of the study. Initially, dissection of the material was largely avoided, to minimize damage to potentially precious museum material. Later, when it was realized that unidentified material would probably be of little value for future research, genitalia were more regularly dissected and examined under a Leitz (Wetzlar) HM-LUX compound microscope at magnifications up to $400 \times$. Sometimes genitalia were cleared by overnight incubation in lactic acid or $10 \% \mathrm{KOH}$, but preferentially they were examined directly in methyl salicylate (wintergreen oil). At lower magnifications, male pedipalps were positioned for examination using a layer of KY jelly under $70 \%$ ethanol, but at higher magnifications in most cases the default positions achieved by placing the pedipalp (dissected at the coxa-trochanter joint) under a cover slip was sufficient for identification. In a few cases, genitalia were stained by brief treatment with $1 \%$ Chlorazol Black in $70 \%$ ethanol to emphasize transparent membranous structures. Specimens were identified where possible using the resources of the Spiders of Europe website (https://www.araneae.nmbe.ch) and the photo galleries of Pierre Oger (https://arachno.piwigo.com) and the Wiki of the German Arachnological Society (https://wiki.arages.de; Lemke et al. 2014). Potential new country records were identified by reference to the Spiders of Europe website (version 03.2019) and the updated checklist of Iberian spiders (Branco et al. 2019), but no efforts were made to identify additional published or unpublished records missing in the database. Sketches of the genitalia are provided for selected specimens, to illustrate the diversity of species collected by Dr. Duffey, to allow verification of difficult cases, and to stimulate other researchers to study Duffey's material and correct the proposed identifications where necessary. All specimens are deposited in the collection of the Manchester Museum. Nomenclature follows the World Spider Catalog (Version 19.5).

\section{List of samples and locations}

Sampling locations were identified using Google Maps, as well as a diverse range of online hiking guides and tourist information websites for some of the more obscure place names. Coordinates are only approximate, as none of the labels contained actual coordinate information. Habitat information and other details follow the original label information as closely as possible; only the spelling of place names has been slightly unified, and abbreviations have been expanded where possible. An overview of the geographical scope of this part of the Duffey collection is shown in Figure 1. 


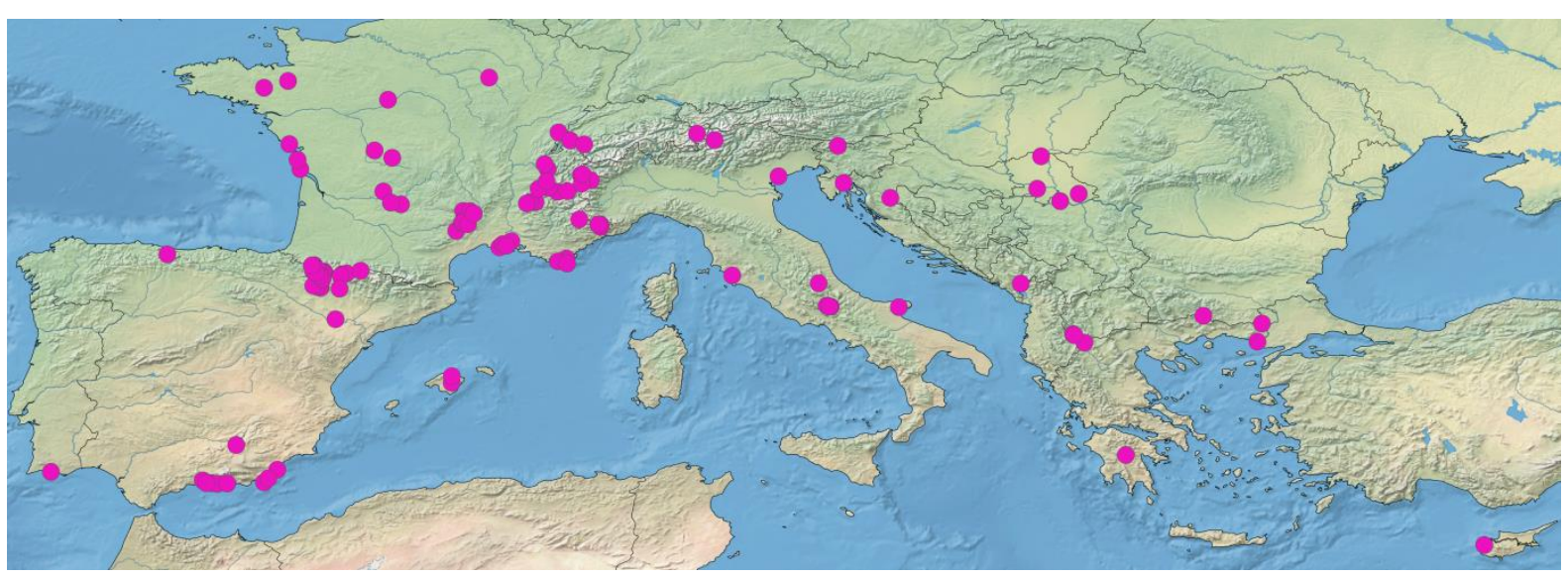

Figure 1. Overview of the South European sampling locations represented in the previously unidentified material of the Duffey collection in the Manchester Museum.

\section{Croatia}

1.- Lovran Istra. 45.296, 14.270

23/Jun/1983. Found crawling on Rita's arm.

2.- Plitvice Lakes National Park. 44.871, 15.598

21/Jun/1983. Collected on rocky walls by lower lakes in National Park.

\section{Cyprus}

3.- - between Ayia and Lysos. 34.998, 32.506

04/Apr/1990. On orchid floret by roadside.

\section{France}

4.- Madone de Fenestre, Mercantour National Park, Alpes-Maritimes. 44.097, 7.356

4a.-14/Sep/1975. 2200 m. Araneus on web in Rhododendron bush. Erigonine under stone in rocky area.

4b. $-14 / \mathrm{Sep} / 1975.2200 \mathrm{~m}$. On webs on rocks. A common spider in this area.

5.- Between Bonneval-sur-Arc and L'Écot, Vanoise National Park, Savoie. 45.375, 7.072 15/Aug/1975. In vegetated scree. Under stones and in grass tussocks. $1800 \mathrm{~m}$.

6.- Between La Grave \& Pic de l'Homme, Écrins National Park, Haute-Alpes. 45.046, 6.127

11/Aug/1975. $1800 \mathrm{~m}$. In wet moss of mountain spring.

7.- Camargue Nature Reserve, Saintes-Maries-de-la-Mer, Bouches-du-Rhône. 43.465, 4.47

7a.- 06/May/1975. Saltmarsh on wet mud and in water under Halimione and Suaeda.

7b.- 08/May/1975. Collected in shrubby Salicornia, grass, Juncus and litter on saltmarsh and margin of sandy islands. Petit Bois des Rièges.

8.- Camargue, Bouches-du-Rhône. 43.557, 4.569

12-18/2/1962. In Juncus and grass in Suaeda and Salicornia marsh of brackish water.

9.- Campsite St. Clair, Le Lavandou, Var. 43.147, 6.38

08/Sep/1975. On van. 
10. - Caston [=Castillon-la-Bataille? Not clear which place is meant]. 12/Jun/2001. In doorway.

11.-Causse de Changefège, Mende, Lozère . 44.502, 3.438 25/May/1975. Under stones in very short vegetation. Scattered bushes.

12.-Causse du Larzac, Aveyron. 43.936, 3.243

12a.- 16/May/1975. In box and under stones in short grass.

12b.- 05/Aug/1975. On flower head and on webs in bushes.

12c.- 13/May/1975. Under stones and in Brachypodium pinnatum between rocks where ground still moist. Nîmes-le-Vieux.

12d.- 13/May/1975. Under stones and running on grass. A few (male Dipoena) in Brachypodium. West of Nîmes-le-Vieux.

12e.- 17/May/1975. In grassland of $15 \mathrm{~cm}$ height. West-facing rocky slope. Above Meyrueis.

13.- Chavigny [not clear which of the places of this name is meant].

25/Jan/1983. Crawling on roadside parking.

14.- Chez Gouillard, Haute-Vienne. 46.222, 0.918

14a.- 14-21/04/2000. Pitfalls near lodge.

14b. - 02/May/2001. Beaten from hedges on east side of étang.

14c.- 02/May/2001. Swept from grass, black knapweed and low Juncus in field where orchids occur.

14d.- 17/May/2001. Beaten from mistletoe on old apple tree and from a few metres of nearby hedge.

14e.- 5-9/04/2002. 6 pitfalls, humid grass between path and mown edge of étang.

14f.- 20/Apr/2002. Beaten from hawthorn, blackthorn, honeysuckle, bramble, holly, oak in hedgerow. Sunny day.

14g.- 30/May/2002. Beating hedge.

14h. - 30/May/2002. Sweeping damp grassland.

14i. - 31/May/2002. Beaten from hedge, west and north boundary.

$14 \mathrm{j}$. - 18/Apr/2003. Collected from hedgerow.

14k.- 18/Apr/2003. Swept from grass and Juncus in fields.

14l.- 15/May/2003. Beaten from south boundary hedge of Chez Gouillard, but from Hugenott side.

15.-Cirque d'Archiane, Drôme. 44.751, 5.498

03/May/1975. In beech litter in open mixed woodland at about $1000 \mathrm{~m}$ altitude.

16.- Cirque de Gavarnie, Hautes-Pyrénées. 42.7, -0.007

02/Aug/1975. Under stones at about $1500 \mathrm{~m}$ within Cirque. Stoney slope, west side, spiny fescue turf.

17.-Cirque de Troumouse, Hautes-Pyrénées. 42.723, 0.118

17a. - 03/Aug/1975. On stony ground by edge of snowbed. Lac des Aires.

17b. - 03/Aug/1975. Under stones and in wet grass and moss by spring.

18.- Col de la Cayolle, Alpes Maritimes. 44.259, 6.744

04/Aug/1982. $2300 \mathrm{~m}$ alt. Under stones in snow and in wet area with Alchemilla alpina.

19.- Col de Loubière, Mont Lozère, Cévennes National Park, Lozère . 44.496, 3.629 
24/May/1975. Under stones on grass. In open grass area in spruce forest, $1150 \mathrm{~m}$.

20.- Col du Chat, Chambéry, Savoie. 45.693, 5.847

26/Apr/1975. leaf litter and moss at about $800 \mathrm{~m}$ in hazel, oak, beech coppice.

21.- Col du Galibier, Hautes-Alpes. 45.064, 6.408

21a. - 13/Aug/1975. $2650 \mathrm{~m}$. Under stones by edge of snow bed. Some still embedded in snow.

21b.- 05/Sep/1975. $2556 \mathrm{~m}$. Collected under stones of snow bed where we collected in mid-August. All snow now gone.

21c. - 05/Sep/1975. 2556m. Collected under stones (near extinct snow bed).

22.- Côte Sauvage, Charente-Maritime. 45.692, -1.197

22a.- 28/May/1966. Pointe de la Coubre. Dunes by edge of pine forest.

22b. - 29/May/1966. Forest ride near road. Running on sandy, sparsely vegetated area.

22c.- 29/May/1966. Near Ronces les Baines. Dunes by forest edge. In pine needles and under plants.

22d.-30/May/1966. Open forest scrub. Mainly swept.

22e.- 01/Jun/1966. Bonne Anse dunes, near Le Coubre. Mainly under "rock rose".

22f._- 01/Jun/1966. Bonne Anse dunes. From mainly grass and Artemisia campestris.

23.- Forêt de Montal, Cévennes National Park, Lozère. 44.162, 3.407

21/May/1975. In wet litter of Molinia and grass in open spring-fed area in spruce/pine, oak.

24.- - Forêt de Rennes, Ille-et-Vilaine. 48.197, -1.546

06/May/1979. Under stone by oldest beech tree in forest.

25.- - Forêt du Prince, Jura. 46.736, 6.154

17/Jun/1989. On field layer plant in forest ride, mainly fir, some beech.

26. - Galoubet, Lot. 44.687, 1.669

13/Jun/2001. Swept from hay field and hedge.

27.- Gordolasque Valley, Mercantour National Park, Alpes-Maritimes. 44.044, 7.356

$27 \mathrm{a}$ - - 15/Sep/1975. End of road at $1820 \mathrm{~m}$. Shaken from grass and litter on slopes above river. Rita C. Duffey coll.

27b. - 15/Sep/1975. End of road at $1820 \mathrm{~m}$. Vegetated scree. Under stones, well below surface stones. Segestria and two with stripey abdomens were under surface stones in vegetated area.

28.- Île d'Oléron, Côte Sauvage, Charente-Maritime. 45.955, -1.274

28a.-31/May/1966. Dunes a few km north of Le Château. In grass and other herbs, some swept.

28b.- 31/May/1966. Dunes des Saumonards, Plage de Plaisance. Running on path through dunes.

29. - La Crau near St. Martin-de-Crau,Bouches-du-Rhône. 43.638, 4.812 10/May/1975. Under stones and in sparse vegetation.

30._- La Pinède, Die, Drôme. 44.757, 5.354

09/Jun/1989. On camping car, evening.

31.-Lac de Trécolpas, Mercantour National Park, Alpes-Maritimes. 44.116, 7.34 13/Sep/1975. $2150 \mathrm{~m}$. Under stones and in grass litter. Alpine grassland around lake. 
32.- Lascaux II, Dordogne. 45.054, 1.168 30/May/1989. In van, parking area in forest.

33.- Le Boréon, Mercantour National Park, Alpes-Maritimes. 44.114, 7.285 13/Sep/1975. $1600 \mathrm{~m}$. Larch forest. Open area of vegetated scree. Under stones in Vaccinium myrtillus, grass, Sedum, Theridiid in Rhododendron ferrugineum.

34. - Les Lanches, Valley of Peisey-Nancroix, Vanoise National Park, Savoie. 45.525, 6.791 20/Aug/1975. Under stones by melting snow and by small spring.

35.- Les Salins, Hyères, Var. 43.067, 6.137 12/Apr/1975. In Agropyron sp. Old salin. Wet ground.

36. - Les Seiglières, Forêt domaniale de Saint-Martin d'Uriage, Isère. 45.156, 5.869 23/Apr/1975. $1065 \mathrm{~m}$. In moss on pine trunk in spruce forest. Smallest was an aeronaut on self while on road.

37.- Longeville-sur-Mer, Vendée. 46.402, -1.509 09/Jun/1964. Consolidated dunes. On blue flower like scabious.

38. - Marais de Lavours, Ain. 45.832, 5.759 26/Apr/1975. Reed, big sedge, Caltha, meadowsweet. Ground wet. Burnt stem stumps.

39.- Meyrueis, Cévennes National Park, Lozère. 44.18, 3.429 39a.- 14/May/1975. $1200 \mathrm{~m}$ altitude. Open beech forest in leaf litter on rocky slope. 2 km south-east Meyrueis Valley, River La Brèze. 39b. - "May 1975". Miscellaneous catch. In caravan or wash place. 39c.- 18/May/1975. Grass and leaf litter under oak scrub and Cytisus purgens. Westfacing slope, River La Brèze, $2 \mathrm{~km}$ south-east Meyruies.

40.- Mont Aigoual, Cévennes National Park, Gard. 44.121, 3.574 40a.- 14/May/1975. $1000 \mathrm{~m}$ alt. In grass and litter under vegetation of Genista purgans.

40b. - 20/May/1975. Under stones and in Nardus sward. All dry apart from 2 Lepthyphantes from damp area. $1500 \mathrm{~m}$ altitude.

41.- Mont Lozère, west of Génolhac, and in area of Belvédère des Bouzèdes, Cévennes National Park, Gard/Lozère.

09/Aug/1975. Pine litter in Vaccinium myrtillus at $1507 \mathrm{~m}$ and beech/pine litter in Deschampsia flexuosa at about $1100 \mathrm{~m}$. Zora and jumpers from 2nd site, apart from 1 small dark.

42.- near Bettu [?]. 27/May/2005. Swept from roadside vegetation.

43. - Near Termignon, Vanoise National Park, Savoie. 45.277, 6.814 14/Aug/1975. Running on stony path. $1700 \mathrm{~m}$. Collected during climb to Lac Blanc de Termignon from Le Villard.

44.- Parc Naturel Régional d'Armorique, Finistère. 48.294, 4.178 05/Jul/1979. Running on wet sphagnum. Several seen.

45.- Parc Naturel Régional du Vercors, Drôme. 45.129, 5.587 45a.- 13/Jun/1989. $1400 \mathrm{~m}$, running on bare earth in area of patchy vegetation. Vallée de Combeau. 45b. - 14/Jun/1989. In crevice on van, origin? 
45c.- 14/Jun/1989. On rock, $1600 \mathrm{~m}$.

46. - Pic de Finiels, Mont Lozère, Cévennes National Park, Lozère . 44.426, 3.739 22/May/1975. 1700 m, under stones and in heather. Mostly Nardus/Vaccinium.

47.— Port Cros, Hyères, Var. 43.004, 6.395

47a.-13/Apr/1975. Segestria in tree crevice, other with two egg sacs in rock crevice. 47b. - 13/Apr/1975. Under stones by path. Maquis vegetation with few trees. East of Fort de l'Estissac.

47c.- 14/Apr/1975. Leaf litter of Holm oak and Aleppo pine forest.

47d.- 17/Apr/1975. In needle leaf litter under large Aleppo pines on steep slope. Le Tuf, half-way down zigzag path.

47e.- 17/Apr/1975. Running in moist meadow by Bay, lightly grazed by two donkeys. Grass height $15 \mathrm{~cm}$.

47f. - 17/Apr/1975. Under isolated plant of Limonium minutum on sandstone cliff. Le Tuf.

47g. - 17/Apr/1975. under piece of driftwood at head of beach. Baie de Port Man. 47h.- 18/Apr/1975. In leaf litter under Holm oak, Erica arborea, Arbutus, Aleppo pine. Slightly moist at $2-3 \mathrm{~cm}$ depth (Theridiid on tree foliage).

47i.- 18/Apr/1975. In thick pine needle leaf litter Aleppo pine. Erica arborea and Arbutus. On way from Baie de Port Man.

47j.- 18/Apr/1975. Salticid female caught eating moth on foliage of Erica arborea 5 ft. height.

48.- Réserve Naturelle de la Tourbière des Dauges, Haute-Vienne. 46.012, 1.419 29/Sep/2001. Sorted from bag of moss from bog.

49.- - Saint-Clair, Le Lavandou, Var. 43.147, 6.38 14/Apr/1975. On caravan.

50.- - Saint-Pierre-de-Chartreuse, Isère. 45.342, 5.815 22/Apr/1975. alt. $900 \mathrm{~m}$.

51. - Southwest of Gourdon, Lot. 44.737, 1.382 12/Jun/2001. Swept from grasses and shrubs on roadside verge.

52.- Station biologique de Paimpont, Paimpont, Ille-et-Vilaine. 48.004, -2.229 52a.- 02/Jul/1979. Two aeronauts by field station. One large agelenid in cell under stone and others swept from Agrostis setacea and Erica cinerea on heath burnt a few years ago. 52b. - 07/Jul/1979. Bathroom. 52c. - 07/Jul/1979. Marsh by lake, sphagnum marshes, vegetation and around tussocks. Very wet.

53.- Tour du Valat, Camargue, Bouches-du-Rhône. 43.512, 4.666 21/Apr/1956. [no further data provided]

54.- Vallée de la Grand-Pierre, Blois, Loir-et-Cher. 47.664, 1.299 10/Jul/1979. Swept from limestone grassland, low bushes of oak, sloe, juniper and box.

55.- Valley of Peisey Nancroix at Lac des Moutons, Vanoise National Park, Savoie. 45.545, 6.833 
19/Aug/1975. All erigonines under stones at 2500-2600 m. Others taken on path below $2000 \mathrm{~m}$.

56.- Vercheny, Drôme. 44.716, 5.252

01/May/1975. Under stone on hillside with open scrub and fine scree. Stones among leaf litter.

\section{Greece}

57.- - [unspecified localities].

"June 1983". Collections from campsite loos and in and on van.

58.- Evros Delta, Thrace. 40.782, 26.047

58a.- 30-31/05-1/6/1983. Caught on van. Evros Marches and on Alexandroupoli.

58b. - 31/05/-1/06/1983. Under Halimione and Salicornia on dry edge of saltmarsh.

59. - Half-way between Protokklision and Mikron Derion, Thrace. 41.298, 26.178

$03 /$ Jun/1983. Collected from grassy vegetation with many plants by roadside.

60.- Mount Mainalo, Falanthos, Arcadia. 37.557, 22.306

13/Jun/1983. At about $1000 \mathrm{~m}$.

61._- Prespa National Park, Mikri Prespa Lake, Macedonia. 40.753, 21.122

16/Jun/1983. Collected under rosettes of Verbascum on sand bar.

62.- - Rhodopi Forest Reserve, Eastern Macedonia. 41.518, 24.511

26-27/05/1983. 1800-1950 m. Collected on ground by paths and in grassy clearings.

\section{Italy}

63.- Camosciara, Abruzzo National Park, L'Aquila. 41.78, 13.906

63a.- 30/Sep/1978. In leaf litter of closed canopy beech wood at $1800 \mathrm{~m}$ on steep slope.

63b. - 30/Sep/1978. In wet moss on rocks by cascade at $1900 \mathrm{~m}$. Open rocky area in beech forest.

64.- Campo Imperatore, Gran Sasso e Monti della Laga National Park, L'Aquila. 42.443, 13.558

07/Oct/1978. $1550 \mathrm{~m}$. Near Gran Sasso under stones in rocky area scanty vegetation.

65.- - Foresta Umbra, Gargano, Foggia. 41.764, 15.84

05/Oct/1978. Quercus cerris leaf litter, mixed with Quercus ilex. In closed canopy woodland.

66.- Isola di Burano, Venice, Venice. 45.486, 12.417 15/Oct/1978. Dunes. In leaf litter under bushes and in lichen and moss. Many immatures discarded.

67.- Maremma Regional Park, Grosseto, Toscana. 42.666, 11.099 18/Oct/1978. Under stones in brackish meadow near coast.

68.- Pineta, Pescasseroli, L'Aquila. 41.808, 13.788 03/Sep/1976. $1220 \mathrm{~m}$, rocky substrate, northeast exposure.

69.- Solda Valley, Stelvio National Park, Trentino. 46.516, 10.597 21/Sep/1978. 1920 m. Under stones on flat plain of Dryas heath. Formed by outwash from mountains.

70.— Val Fondillo, Abruzzo National Park, L’Aquila. 41.779, 13.856 


\section{Macedonia}

71._ Gradište campsite, Peštani, Ohrid. 40.996, 20.799 20/May/1983. Swept from herbs and bushes, very small spider was under stone.

72.- National Park Galičica, Trpejca, Ohrid. 40.968, 20.837 22/May/1983. 1600m. Swept \& collected on ground. Road.

\section{Montenegro}

73.- Podgorica (Titograd). 42.434, 19.314

18/Jun/1983. Rocky slopes with scrub and scattered plants.

\section{Portugal}

74.- Algarve. 37.079, -8.296 Spring 1980. On shrub. Died 10.10.1980.

\section{Serbia}

75.- Belgrade. 44.779, 20.434 15/May/1983. In van on campsite northwest side of city.

76. - Near Devojački Bunar, Deliblatska Peščara Special Nature Reserve. 44.988, 20.963 16/May/1983. Swept from dry grassland.

77.- Near Male Pijace, Subotica. 46.055, 19.889 14/May/1983. Dry steppe grassland.

78. - Near Vrdnik, Fruška Gora National Park . 45.132, 19.779 15/May/1983. On dry grassland. Swept.

\section{Slovenia}

79.- - Triglav National Park. 46.353, 14.107 12/Sep/1980. $1100 \mathrm{~m}$ altitude on plateau north of National Park. Under bark in Picea forest.

\section{Spain}

80. - 1700m above Puento del Ruso, Somport, Huesca. 42.774, -0.519 02/Jun/1973. On grass or in Vaccinium of Pinus uncinata forest.

81. - $20 \mathrm{~km}$ east of Nerja, Málaga. 36.758, -3.715

17/Mar/1979. Under stones in garigue vegetation on hillside near coast.

82.- About $1 \mathrm{~km}$ northeast of lighthouse and $15 \mathrm{~km}$ southwest of Almería. 36.781, -2.23 22/Mar/1979. Saltmarsh, under fronds of shrubby Salicornia.

83.- Ansó Valley, Huesca. 42.784, -0.828

83a.- - 04/Jun/1973. In moss \& leaf litter, beech wood four $\mathrm{km}$ north of village. 83b. - 04/Jun/1973. Under stones and in leaf litter just north of village. 83b. - 04/Jun/1973. Under stones in leaf litter under beech just north of village. 83c. - 05/Jun/1973. Taxeres. Mostly under rotten wood. Trunks of beech and under bark. A few running or under stones.. 
83d.- 05/Jun/1973. Taxeres. Rotten wood, under bark etc. in beech. A few taken running and under stones.

83e.- 06/Jun/1973. Between Ansó and Valle de Roncal. At highest point. Remainder of collection from lichen on scots pine.

83f._ 06/Jun/1973. Highest point between Ansó and Valle de Roncal. Shaken from moss around scots pine.

83g.- 10/Jun/1973. Under stones and in beech leaf litter.

83h.-21/Sep/1974. General beating.

83i.- 21/Sep/1974. Under stones in beec h litter on valley side. Leptoneta site. 83j. - 23/Sep/1974. Dry Barranci. Under stones.

84.- Barranco de Agüerri, Huesca. 42.792, -0.695

30/May/1973. Under stones of erosion slope. South side of river.

85.- Belagua, Huesca. 42.957, -0.802

29/May/1977. Vacuumed from dry grassland.

86. - Between Puerto de Motril and Solbreña, Granada. 36.739, -3.556

18/Mar/1979. Collected under stones, driftwood, rubbish etc. at head of beach.

87.- Castillo de Loarre, Huesca. 42.326, - 0.612

26/May/1977. Vacuumed from dry grassland.

88. - Ena-Ordaniso, Huesca. 42.448, -0.694

88a.- 27/Jun/1972. On ground in herbaceous vegetation and under stones amongst small pine trees.

88b. - 24/Sep/1974. Grassland with pine near the road barrier. In grass and leaf litter. 88c. - 24/Sep/1974. In grass, pine needles, under wood, leaf litter. In open pine with grass near road barrier. Rita C. Duffey coll.

88d.- 24/Sep/1974. In leaf litter and among wood fragments beyond road barrier. p.m.

88e.- 24/Sep/1974. In leaf litter and grass near barrier - mostly beyond it. p.m. Rita C. Duffey coll.

88f. - 24/Sep/1974. Just before barrier. Spiders from D-vac. collection of grassland and herbs among pine trees.

88g. - 25/May/1977. Vacuumed dry grassland.

89.- Hills around Laguna Ercina, Covadonga National Park, Asturias. 43.267, -4.981

18/Jul/1975. Under stones and around tussocks of vegetation.

90.- Hills near Frigiliana, Nerja, Málaga. 36.792, -3.892

90a.- 19/Mar/1979. Under stones in garigue (burnt 3 years ago). On bushes (large spiders).

90b.- 21/Mar/1979. In sheltered valley well vegetated with shrub. Under stones in patches of scree in macquis.

91.- Jaca, Huesca. 42.564, -0.572

91a._- "June 1972". [no further data provided]

91b. - 20/Jun/1972. Camping Victoria. Swept from tall herbs.

91c. - 20/Jun/1972. Camping Victoria. Taken on building of camp site.

91d.-23/Jun/1972. In pine/holly leaf litter.

91e._- "1973". [no further data provided]. 
92._L La Mamola, Granada. 36.747, -3.277 22/Mar/1979. Under stones, around rosette plants and the Araneus on web in rock cliff. All by coast.

93._L Laguna La Playa, Bujaraloz, Zaragoza, Spain. 41.42, -0.194 93a.- 11/Jul/1975. Dry inland salt area in leaf litter under shrubby Salicornia etc. 93b.- 11/Jul/1975. Immatures. In leaf litter and shaken from shrubby Salicornia \& Artemisia on dried out inland saltmarsh. 93c.- 11/Jul/1975. In dry leaf litter under shrubby Salicornia, on bed of dry salt lake.

94.- Las Blancas, Huesca. 42.715, -0.564 $01 / \mathrm{Jul} / 1972$. Under stones at $2000 \mathrm{~m}$. Alpine tundra.

95.- Las Tiesas Bajas, Huesca. 42.609, -0.660 27/May/1973. Running on dry stone shingle banks by Rio Estarrún.

96.- - Linza, Huesca. 42.898, -0.800 96a.- 03/Jul/1972. In beech leaf litter near head of valley. 96b. - 05/Jun/1973. Leaf litter, beech wood. 96c. - 23/Sep/1974. In grass on side of small valley. 96d. - 24/May/1977. Vacuumed from dry grassland. 96e. - 28/May/1977. Vacuumed from dry grassland.

97.- Loarre, Huesca. 42.314, -0.626 97a.-30/Jun/1972. Grubbing in grass and moss under pine and box. 97b.- 30/Jun/1972. Swept from tall herbs, grass and low bushes by box/pine wood. 97c. - 19/Sep/1974. In moss \& leaf litter under box \& pine.

97d. - 19/Sep/1974. In moss and leaf litter under box and pine (same as 1972 site). 97e.- - 19/Sep/1974. Under stone on hillside with box.

98. - Los Lecherines, Huesca. 42.744, -0.539 20/May/1977. Vacuumed from dry grassland.

99.- Mallos de Riglos, Huesca. 42.351, -0.724 29/Sep/1974. About 800m. Under stones in Schoenus and Juncus area.

100.- Mallos de Riglos, Huesca. 42.351, -0.724 30/Sep/1974. On rock. Fed in lab throughout October at Monks Wood. Found dead 31 October. Still immature.

101.- Mojácar, Almería. 37.141, -1.85 01/Feb/1983. Catch from 4 pitfalls put out on hillside behind campsite.

102.- Molino de Aratores, Huesca. 42.655, -0.556 20/Sep/1974. Thin layer of wet moss over mud in Juncus. Rita C. Duffey coll. 103.- Monte El Boalar, Huesca. 42.558, - 0.636 103a.- 24/Jun/1972. In leaf litter under willow by Rio Aragón. 103b. - 24/Jun/1972. In leaf litter under willow by Rio Aragón. 103c. - 24/Jun/1972. Swept from vegetation on shingle by Rio Aragón. 103d.-24/May/1973. Running on shingle by stream. 103e.- 27/May/1973. Beaten from foliage of oak, hawthorn, juniper, box, honeysuckle and other shrubs. 103e2.- 27/May/1973. Beaten from oak, hawthorn, juniper, box, honeysuckle and other shrubs in woodland. 
103f. - 27/May/1973. Swept from grass and flowering herbs in strip of meadow just below wood. 103g. - 27/May/1973. Swept from hawthorn blossom. 103h. - 24/Jun/1974. Swept from vegetation on shingle by Rio Aragón. 103i. - 16/Sep/1974. Beaten from oak. 103j. - 16/Sep/1974. Collected from wet leaf litter and grass under oak. 1 hour. Rita C. Duffey, coll. Raining during collecting. 103k.- 16/Sep/1974. Collected from wet leaf litter and grass under oak. Raining during collecting. 1 hour.

1031. - 16/Sep/1974. Shingle bed of Rio Aragón. Around plants and shrubs litter. Rita C. Duffey, coll.

103m. - 16/Sep/1974. Shingle bed of Rio Aragón. On plants and in lead litter around base of plants and willow shrub.

103n.- 25/Sep/1974. Collected from bag of moss and leaf litter taken from under box and oak .

103o. - 25/Sep/1974. Grubbed from grassland in open area of woodland.

103p. - 25/Sep/1974. Immatures from leaf litter and moss and from grass litter. Open deciduous woodland.

103q. - 25/Sep/1974. Swept and grubbed in grassland in box and oak. Immatures. 103r. - 28/Sep/1974. From leaf litter and moss under box, pine and oak.. 103s. - 18/May/1977. Vacuumed from dry grassland.

104. - Near Cómpeta, Nerja, Málaga. 36.835, -3.972 25/Mar/1979. Stony steep hillside, with olives and scattered scrubs. Very little herbaceous vegetation.

105.- Near Níjar, Almería. 36.895, -2.107

04/Feb/1983. In short grass on stoney hillside.

106. - near Petra, Mallorca. 39.613, 3.113

17/Apr/1991. Under clod of earth in abandoned agricultural land.

107.- - Nerja, Málaga. 36.758, -3.871 107a. - 26/Mar/1979. In hills at back of town. Under stones, shrubs and grass tussocks. Stony hillside with Cistus and rosemary, few herbs present. 107b. - 29/Mar/1979. In low hills at back of town. Macquis vegetation but very green because of recent rain. Under stones.

108. - North of Aragüès, Huesca. 42.729, -0.678 03/Jun/1973. 1400m, swept and beaten in open woodland where road ends. Lycosids on ground.

109.- - Ordesa National Park, Huesca. 42.656, -0.049 27/Sep/1974. Pine leaf litter.

110._- Ordesa National Park, Huesca. 42.656, -0.049 110a. - 27/Sep/1974. Shaken from beech leaf litter in shade. 110b. - 09/Jul/1975. $2000 \mathrm{~m}$. North-facing side of valley. Under stones and in ungrazed alpine meadow with cranesbill, cornflower, Gentiana lutea, Trollius. Dry.

111.- - Puente del Ruso, Huesca. 42.798, 0.521 22/May/1977. Vacuumed from dry grassland. 
112.— Riglos, Huesca. 42.348, -0.725 29/Sep/1974. Swept from roadside shrubs. Rosemary, oak (with holly-like leaves) and indetermined shrub with red berries.

113.- Rodellar, gorge of Rio Mascún, Huesca. 42.290, -0.080 113a. - 08/Jun/1973. In moss, under stones, swept. 113b. $-08 / \mathrm{Jun} / 1973$. Shaken from moss. 113c. - 08/Jun/1973. Swept and beaten and on ground. 113d.- 08/Jun/1973. Swept, beaten, on ground and shaken from moss. 113e.- 08/Jun/1973. Swept, beaten, under stones.

114. - S'Albufera Natural Park, Mallorca. 39.802, 3.117 20/Apr/1991. Taken on reed heads or swept from low reed.

115.- San Felices, Huesca. 42.375, -0.827 115a.- 07/Jun/1973. Sweeping and beating along road from Agüero to San Felices. $115 \mathrm{~b}$. - 07/Jun/1973. Under stones in moist conifer leaf litter. Theridion on web on conifer.

116. - San Juan de la Peña, Huesca. 42.566, -0.561 116a.-23/Jun/1972. Leaf litter in holly/pine wood. 116b. - 24/Jun/1972. Running on short open turf. 116c. - 26/Jun/1972. At monastery. 116d._-26/Jun/1972. Meteorological station. In open grassy area with pine trees. 116e.-26/Jun/1972. Meteorological station. Swept from grassy area with flowers. 116f.- 25/May/1973. On and in lichen on scots pine. 116g. - 25/May/1973. Running on \& under stones at the Solana. 116h.- 25/May/1973. Running on ground in pine, beech, Abies, holly, box woodland.

116i.-25/May/1973. Shaken from moss around base of pine trees in mixed forest. 116j.-26/May/1973. Beaten from beech.

116k.- 26/May/1973. Collected from short wet vegetation around pond at monastery.

1161.-26/May/1973. Swept from buxus.

116m.-26/May/1973. Swept from foliage of pine, box, juniper, beech and Abies. 116n. - 12/Sep/1974. Grubbing in wet vegetation and litter around pond by monastery. 1 hour. Rita C. Duffey, coll.

116o.-30/May/1977. Vacuumed from dry grassland. Estación Meteorológica. 116p.-26/Jun/1977. Running in grassy area amongst pines. Estación Meteorológica.

117.- - Selva de Oza, Huesca. 42.834, -0.711 117a.- 18/Sep/1974. About 1200m. Wet grass, sedges and mosses by side of small stream, down grassy hillside. 1 hour.

117b. - 18/Sep/1974. About 1200m. Wet grass, sedges and mosses growing by side of small stream down grassy mountainside. About 1 hour. Rita C. Duffey, coll.

117. - Selva de Oza, Huesca. 42.834, -0.711 $117 \mathrm{c} .-18 / \mathrm{Sep} / 1974$. Casual under stones and in rotting wood of old beech trees.

118.- Sierra de Cazorla, Jaén. 37.841, -3.021 "May 1986". [no further data provided] 
119.- Sierra Nevada, Granada. 37.841, -3.021 27/Mar/1979. 1700-2200 m. Under stones by roadside and on hillside on road to Parador de Sierra Nevada.

120.- Sinués, Huesca. $42.654,-0.649$ 120a. - 22/Jun/1972. In pine wood. 120b. - 26/Jun/1972. Under stone in vegetated bottom of river bed. 120c. - 17/Sep/1974. Adults from grass litter and from under stones in grass. Edge of river bed of Río Estarrún below road.

120d.- 17/Sep/1974. Grass tussocks on sand and shingle surrounded by bushes. Río Estarrún.

120e.- 17/Sep/1974. In grass tussocks on sand and shingle near bushes. Río Estarrún.

120f. - 17/Sep/1974. In grass tussocks on sand and shingle. Río Estarrún.

121. - Valle de Hecho. 42.737, -0.749

121a.- 18/Sep/1974. Sorted from back of leaf litter from box and beech. Hillside with mixed forest between Hecho and Selva de Oza..

121b.- 28/May/1973. Between Hecho and Selva de Oza. Beaten from foliage of beech, pine, Abies, box, juniper \& other shrubs.

121c. - 28/May/1973. Between Hecho and Selva de Oza. Beech litter.

121d.- 28/May/1973. Between Hecho and Selva de Oza. Swept from foliage of beech, pine, Abies, box, juniper and other shrubs.

121e.-29/May/1973. Alpine pastures of Guarrinza, under stones at about 1450m.

121f. - 29/May/1973. Collected under stones at about 1600m, in region of Guarrinza by entrance of small valley with tourbière.

121g.- 29/May/1973. Guarrinza, 1400m. Under stones and running on grass on alpine meadows.

121h. - 29/May/1973. Near Guarrinza, collected from wet vegetation in spring on hillside.

121i. - 29/May/1973. Near the Col of Anzotiello, 1600m. Under stones on alpine pastures.

121j.- 29/May/1973. Under stones and running on grass. 1400m. Alpine pastures in region of Guarrinza.

121k. - 30/May/1973. Calzada Romana. Swept, beaten, under stones and running.

1211. - 30/May/1973. In soaking wet moss on stones of mountain torrent.

121m. - 30/May/1973. Loo at campsite.

121n. - 30/May/1973. swept and beaten from bushes and field layer.

121o. - 31/May/1973. Under stones on grassy hillside.

121p.- 21/May/1977. Vacuumed from dry grassland.

122.- Venta de Arraco, Navarra. 42.947, -0.868

122a._ "June 1972". [no further data provided]

122b. - 28/Jun/1972. In beech leaf litter.

122c. - 21/Sep/1974. 1600-1700m. Under stones and in Festuca scoparia grass. 122d.- 21/Sep/1974. Spiny Festuca cut from around rocks. Open Pinus uncinata forest. 


\section{Switzerland}

587 123.- Campsite, Morges, Vaud. 46.504, 6.489 31/Aug/1975. Aeronauts.

124.- Gros Brasset, Vaud. 46.395, 6.884 02/Sep/1975. Wet marsh litter, but mostly from sandy beach and grass tussocks.

\section{Species list with annotations}

\section{Agelenidae}

596 Allagelena gracilens (C. L. Koch, 1841) 103m (1ㅇ)

597 Coelotes atropos (Walckenaer, 1830) $34(29)$

$598 \quad$ Coelotes poweri Simon, $1875 \quad 31(1$ + $)$

599 Coelotes terrestris (Wider, 1834) $24(1$ )

600 Eratigena bucculenta (L. Koch, 1868) s. lat. (morph C) $88 \mathrm{~d}(1$ ) $\quad$ Figure 2A

601 This specimen is clearly distinct from E. bucculenta as illustrated by Machado (1941), 602 Brignoli (1978) and Bolzern et al. (2013); most importantly, the copulatory ducts are 603 considerably longer. However, this is in good agreement with the females illustrated by 604 Bolzern et al. as E. bucculenta s. lat. (morph C; e.g., Bolzern et al. 2013:fig. 11P). Of course, 605 the question whether this form is an independent species can not be answered based on this 606 single specimen, but the discrete variation seems to exceed what would typically be expected 607 for conspecific material.

608

609 Eratigena feminea (Simon, 1870) $119(1 ð) \quad$ Figure 2B

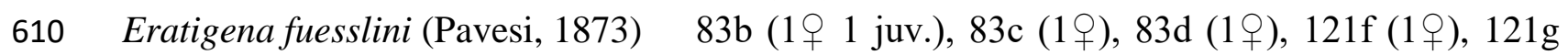
611 (1 $), 121 \mathrm{k}(1+1 \precsim) \quad$ Figure 2C, D

612 Lycosoides coarctata (Dufour, 1831) 81 (1ㅇ), $90 \mathrm{~b}(1+$ )

613 Malthonica lusitanica Simon, 1898 83b (2q), 83f (4q), 83g (6q), 88b (1 $q$ ), 91d (10q 3

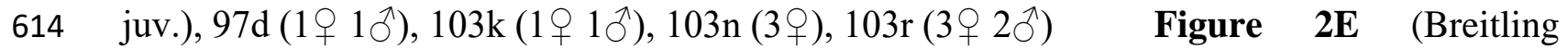
615 2018:figure 2L)

616 Pireneitega segestriformis (Dufour, 1820) $83 \mathrm{i}(1+$ ) $\quad$ Figure 2F

617 Textrix caudata L. Koch, $1872 \quad 119(1$ + $)$

618 Textrix denticulata (Olivier, 1789) 83d (1q2 juv.)

\section{Amaurobiidae}

621 Amaurobius erberi (Keyserling, 1863)

$$
47 \mathrm{c}(1 q), 47 \mathrm{~g}(1 \text { + }), 47 \mathrm{i}(1 q)
$$

623 Anyphaenidae

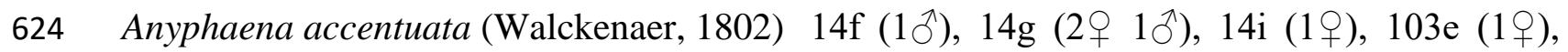
$625121 \mathrm{~b}(1+1$ juv.)

626 Anyphaena sabina L. Koch, 1866 47h (2 juv.), $71(1+), 103 \mathrm{f}(1+) \quad$ Figure 3 


\section{Araneidae}

629 Aculepeira carbonaria (L. Koch, 1869) 4b (29)

630 Agalenatea redii (Scopoli, 1763) 12a (1ठ), 14c (2), 14k (1워), 39b (1), 113c (1)

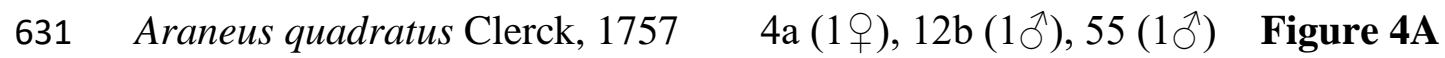

632 Araneus sturmi (Hahn, 1831) 116k (1ठ) Figure 4B

633 Araneus triguttatus(Fabricius, 1775) 103e (2q)

634 Araniella alpica (L. Koch, 1869) 25 (1우 1 ठ)

635 Araniella cucurbitina (Clerck, 1757) 2 (1ठ), 22d (2ठ), 108 (1 + ) $), 115 \mathrm{a}\left(1 \circlearrowleft^{\Uparrow}\right)$

636 Araniella inconspicua (Simon, 1874) 103e2 (1ㅇ)

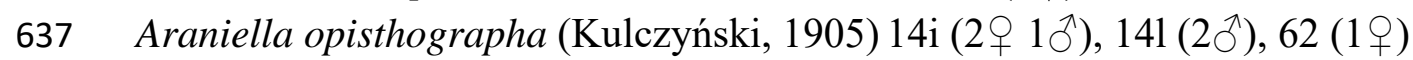

638 Cercidia prominens (Westring, 1851) 12e (1)

639 Cyclosa algerica Simon, $1885 \quad 12 \mathrm{~b}(1+), 89(2+) \quad$ Figure 5A, B

640 Cyclosa conica (Pallas, 1772) $108\left(1 \delta^{\Uparrow}\right), 113 \mathrm{c}(1+$ ) $), 121 \mathrm{k}(1 \bigcirc 1 \overbrace{}^{\Uparrow})$ Figure 4C, 5C

641 Gibbaranea bituberculata (Walckenaer, 1802) 116j (1q)

642 Gibbaranea gibbosa (Walckenaer, 1802) 14d (1q 1ठ), $141\left(1 \delta^{\Uparrow}\right), 71\left(1{ }^{\Uparrow}\right), 121 \mathrm{k}\left(1{ }^{\Uparrow}\right)$

$643 \quad$ Figure 4D, E

644 Hypsosinga albovittata (Westring, 1851) 54 (29), 121p (4 juv.)

$645 \quad$ Hypsosinga heri (Hahn, 1831) $54(1$ )

646 Hypsosinga pygmaea (Sundevall, 1831) 14k (1ठึ)

647 Hypsosinga sanguinea (C. L. Koch, 1844) 54 (1우), 103s (1ठ), 116o (1ㅇ), 121k (1ठ), 121n

$648 \quad(1 q) \quad$ Figure 4F

649 Larinioides cornutus (Clerck, 1757) 14c (1ठ), 14h (1ठ), 14k (1ठ)

650 Larinioides suspicax (O. P.-Cambridge, 1876) $8(1$ ) $), 114(1 \precsim) \quad$ (Breitling

651 2018:figure 2G)

652 Leviellus kochi (Thorell, 1870) 113c (19)

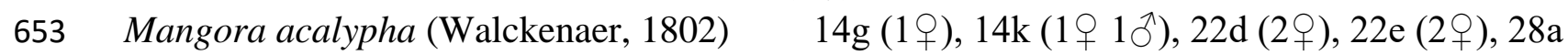

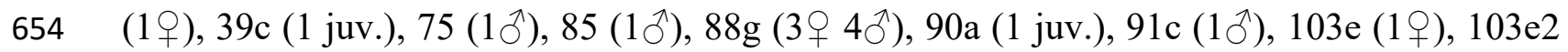

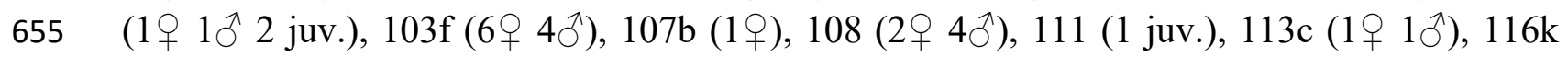

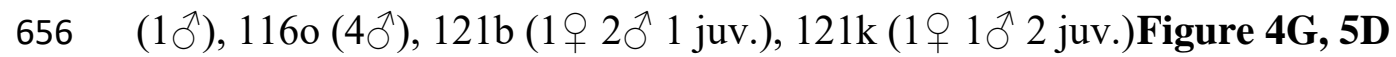

657 Nemoscolus laurae (Simon, 1868) (Simon, 1868) 22f $(1 \overbrace{}^{\Uparrow}), 95\left(1{ }^{\Uparrow}\right)$

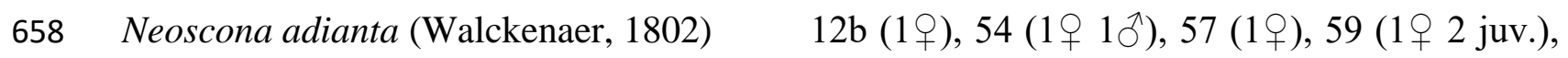

$65989(1$ ㅇ)

$660 \quad$ Neoscona subfusca (C. L. Koch, 1837) 92 (1q)

661 Zilla diodia (Walckenaer, 1802) 103e2 (1 juv.), 113a (1甲), 113c (2甲), 115b (1 9 )

662 Zygiella $x$-notata (Clerck, 1757) $66(1$ )

663

664 Cheiracanthiidae

665 Cheiracanthium elegans Thorell, $1875 \quad 26(1$ q $)$

666 Cheiracanthium erraticum (Walckenaer, 1802) 57 (1ㅇ)

667 Cheiracanthium pennyi O. P.-Cambridge, $1873 \quad 78(4 \bigcirc 3$ Љ)

668 Cheiracanthium striolatum Simon, 1878 22c (1ㅇ), 22e (1ठ), 90b (1 juv.), 107a (1ㅇ)

669 (Breitling 2018:figure 2F)

670 


\section{Clubionidae}

672 Clubiona brevipes Blackwall, 1841 14i (1ठ), 103e (1ठ), 103e2 (1q), 113a (1q), 113c $673(1$ q $), 121 \mathrm{k}(1 \overbrace{}^{\Uparrow})$

674 Clubiona comta C. L. Koch, 1839 14b (4ㅇ), 14d (1), 103e (1우), 103r (1ㅇ), 108 (1우), $675113 \mathrm{a}\left(1+1{ }^{\Uparrow}\right), 1161\left(1 \delta^{\Uparrow}\right), 116 \mathrm{~m}(1+), 121 \mathrm{~b}(4+$ ㅇ $)$

676 Clubiona diversa O. P.-Cambridge, 1862 96c (6ㅇ 4ð), 111 (1ㅇ)

677 Clubiona neglecta O. P.-Cambridge, $1862 \quad 8$ (1ㅇ)

678 Clubiona pallidula (Clerck, 1757) 14i (1)

679 Clubiona phragmitis C. L. Koch, $1843 \quad 114$ (2q)

680 Clubiona pseudoneglecta Wunderlich, 199454 (1q)

681 Clubiona subtilis L. Koch, $1867102(2$ ㅇ 1 đ̂)

682 Clubiona terrestris Westring, 1851 14g (1ठ), $14 \mathrm{i}$ (1q), 65 (1), 103r (1q), 121b (2ᄋ), $683 \quad 121 \mathrm{~d}(2+) \quad$ Figure 6

684 Porrhoclubiona vegeta (Simon, 1918) 22a (1థ), 22e (1つ)

685

686

687

\section{Cybaeidae}

688

689

690 Dictynidae

Cryphoeca silvicola (C. L. Koch, 1834) 63a (2)

\section{Cybaeus raymondi (Simon, 1916) 83i (1ㅇ) NEW FOR SPAIN}

691 Archaeodictyna ammophila (Menge, 1871) 29 (2q)

692 Brigittea latens (Fabricius, 1775) $\quad 58 \mathrm{a}\left(1 \circlearrowleft^{\Uparrow}\right), 121 \mathrm{~b}(1 \overbrace{}^{\Uparrow}), 121 \mathrm{k}(1+) \quad$ Figure 7A

693 Dictyna uncinata Thorell, 1856 14d (2ㅇ 3ð), 14i (1우), 59 (1우)

694 Lathys heterophthalma Kulczyński, 1891 97c (19) NEW FOR MAINLAND SPAIN

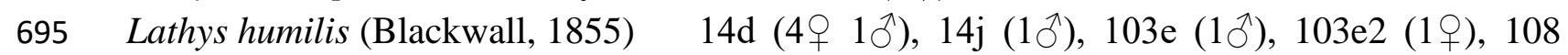

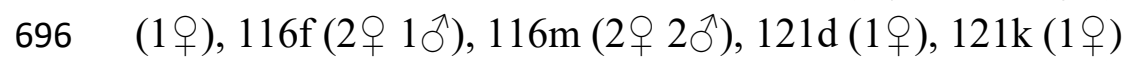

697 Lathys stigmatisata (Menge, 1869) 22c (5ㅇ), 22e (3ㅇ), 28a (6ㅇ), 46 (1ㅇ)

698 Marilynia bicolor (Simon, 1870) 103c (1ठํ) (Breitling 2018:figure 2R)

699 Nigma gratiosa (Simon, 1881) 103e $(1 \precsim), 115 \mathrm{a}\left(1 \circlearrowleft^{\Uparrow}\right)$ Figure 7B (Breitling 2018:figure

700 2D)

701 Two male specimens of this rarely illustrated species were found in Duffey's material. 702 Unfortunately, a female specimen collected at the same site turned out to belong to N. puella 703 (see below; also compare illustration in Crespo et al. 2018).

Nigma hortensis (Simon, 1871) 91b (1ठ゚)

707 (19), 115a $(2 \precsim) \quad$ Figure 7C

$708 \quad$ (Scoto)Lathys sp. $104(1$ ) $\quad$ Figure 7D, 8A

709 This single specimen could not be identified with confidence. The absence of the anterior 710 median eyes could indicate a placement in Scotolathys, but the habitus and genitalia would 711 seem to support a placement in Lathys, close to L. narbonensis. The loss of the AMEs could 712 in this case be an individual variation, rather than indicating a new species.

\section{Dysderidae}


717 Dysdera ninnii Canestrini, $1868 \quad 65(19)$

718 Harpactea corticalis (Simon, 1882) 47c (1ठ)

719 Harpactea hombergi (Scopoli, 1763) 83f (1웅), 103r (1ठ), 113a (1q), 113b (3ㅇ 1ठ),

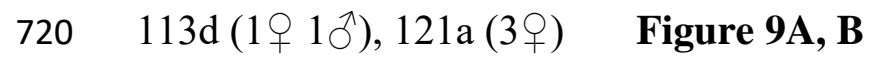

721 Harpactocrates ravastellus Simon, 1914 83i (1仓), 96b (2仓), 110a (3ᄋ), 122b (1ठ) $722 \quad$ Figure 9C, D

723 These specimens correspond morphologically to the genitalic type B described by Bidegaray724 Batista et al. (2016:fig.7), as would be expected based on the known distribution of this morphotype in the Western and Central Pyrenees. They may thus represent a vicariant sister species of $H$. ravastellus s. str.

Parachtes teruelis (Kraus, 1955)

$88 \mathrm{~b}\left(1{ }^{\Uparrow}\right), 88 \mathrm{c}\left(2{ }^{\Uparrow}\right), 103 \mathrm{k}\left(1{ }^{\Uparrow}\right)$

Figure 9E

\section{Eresidae}

Eresus sandaliatus (Martini \& Goeze, 1778) 71 (1ð)

\section{Eresus cf. solitarius Simon, $1873 \quad 116 \mathrm{~g}(1 \overbrace{}^{\Uparrow}) \quad$ Figure 8B, 10A}

In the absence of a modern revision of the Iberian representatives of Eresus, complementing the work of Řezáč et al. (2008) on Central European species, a confident identification of this specimen is not possible at this moment. Both the shape of the conductor and the colouration could indicate that it also belongs to the widely distributed $E$. sandaliatus, which however has not yet been reported from Spain or the adjacent areas of France, while several endemic species are poorly known. Amongst these, E. solitarius seems to show the closest similarity (Bacelar 1936), but this putative identification is suggested with even greater hesitation than for other similar cases presented here.

Stegodyphus lineatus (Latreille, 1817)

$74(19), 90 \mathrm{a}\left(2 \bigcirc 1{ }^{\Uparrow}\right)$

Figure 10B-D

\section{Filistatidae}

Filistata insidiatrix (Forsskål, 1775) 107a (4ㅇ), 113c (19 1 juv.)

\section{Gnaphosidae}

Aphantaulax cincta (L. Koch, 1866) 115a (1ठ)

Civizelotes medianus (Denis, 1936) 113c (19)

Drassodes albicans (Simon, 1878) 113e (19)

Drassodes fugax (Simon, 1878) 110b (4ㅇ) , 122d (1)

Drassodes lapidosus (Walckenaer, 1802)２2a (1ๆ 1ð), 29 (2q 1ð), 58b (1q), 113a (1ठ), $113 \mathrm{c}\left(1 \circ 1{ }^{\Uparrow}\right), 121 \mathrm{~g}\left(1{ }^{\Uparrow}\right)$

Figure 11A

Drassodes pubescens (Thorell, 1856)

$1160(19)$

Drassodex fritillifer (Simon, 1914) $99(1$ ふ)

Figure 11B, C

Drassodex hispanus (L. Koch, 1866) 116g (1q) Figure 12A

Gnaphosa occidentalis Simon, 1878 52a (1q)

Figure 12B

Gnaphosa tigrina Simon, 1878

$121 \mathrm{f}(1$ ㅇ)

Figure 12C 
This female specimen was found in late May, much earlier than most literature records for this species, which refer to collections of mature females in July and August. The identification is based on the illustrations provided by Grimm (1985) - Siberian records assigned to this species, illustrated by Tuneva \& Esyunin (2003), are unlikely to be conspecific.

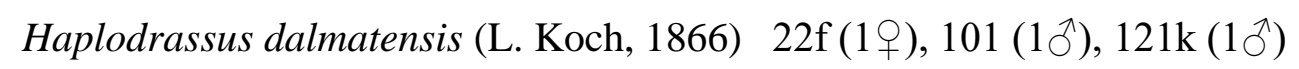

Haplodrassus lyndae Abrous \& Bosmans, $2018 \quad 1031\left(1 \delta^{\widehat{\lambda}}\right) \quad$ Figure 11D, E

Haplodrassus rufipes (Lucas, 1846) 107a $\left(1 \delta^{\Uparrow}\right) \quad$ Figure 11F, G

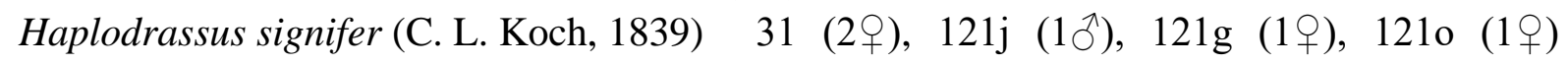
Figure 11H, I

Haplodrassus typhon (Simon, 1878) 120a (1ㅇ)

The genus Haplodrassus is an excellent example of a difficult group, in which Iberian material would have been impossible to identify to species level before the recent revision by Bosmans et al. (2018).

Micaria aenea Thorell, $187162(1$ ふ)

Micaria dives (Lucas, 1846) 22c (1), 22e (19), 22f(1), 28a (1ㅇ)（Breitling 2018:figure 2S)

Micaria formicaria (Sundevall, 1831) 43 (1ㅇ)

Micaria pulicaria (Sundevall, 1831) 62 (1ㅇ)

Micaria rossica Thorell, 1875

Micaria silesiaca L. Koch, $1875 \quad 46$ (1우)

Nomisia exornata (C. L. Koch, 1839)121k (1 )

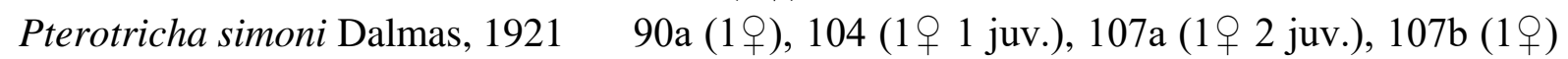

\section{Figure 12D}

Sernokorba tescorum (Simon, 1914) 88g (1ठ) (Breitling 2018:figure 2C)

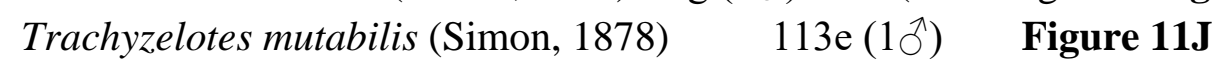

Urozelotes rusticus (L. Koch, 1872) 10 (10)

Zelotes fulvopilosus (Simon, 1878) 88g (1 ㅇ)（Breitling 2018:figure 2J)

Zelotes gallicus Simon, 1914 22a (1우)

Zelotes latreillei (Simon, 1878) $\quad 121 \mathrm{~g}(1+) \quad$ Figure 12E

Zelotes longipes (L. Koch, 1866) 22c (19)

Zelotes manius (Simon, 1878) $1031(1 \overbrace{}^{2}), 116 \mathrm{~g}(1$ \% $)$ Figure 11K, 12F

Zelotes thorelli Simon, 1914 115b (1)

\section{Hahniidae}

Antistea elegans (Blackwall, 1841) $55(1 \overbrace{}^{\lambda}), 83 \mathrm{~d}(1+$ ) $), 102\left(3 q 2 \bigcirc^{\Uparrow}\right), 121 \mathrm{j}(1+$ ) Figure 13A Chorizomma subterraneum Simon, $1872 \quad 83 \mathrm{~g}(2$ )

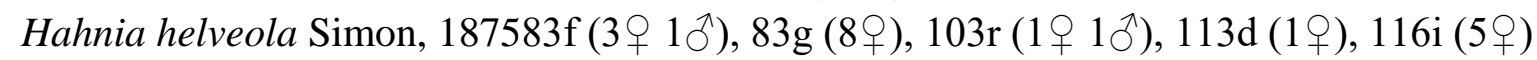

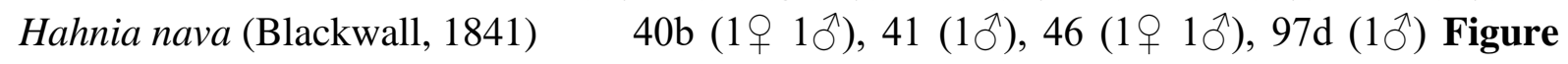
13B

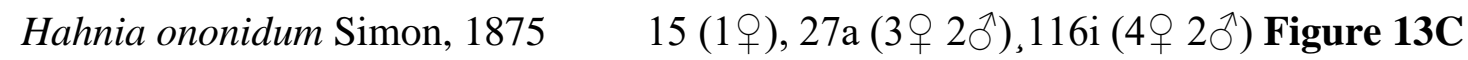


Hahnia petrobia Simon, 1875 $113 \mathrm{a}(1 \circ), 115 \mathrm{~b}(1 \circ)$

Iberina candida (Simon, 1875) 103k (1ㅇ), 121a (1ठ)

Iberina montana (Blackwall, 1841) 83b (2ㅇ), 83g (15ㅇ), 122c (1)

\section{Hersiliidae}

Hersiliola cf. simoni (O. P.-Cambridge, 1872) $\quad$ 107b (1甲) $\quad$ Figure 14

This single female specimen is identified only with some reluctance. The copulatory duct shows clearly fewer coils than illustrated, e.g., by Wiehle (1960:fig. 15, sub H. lucasi), Ribera et al. (1988:figs. 9 and 10), Levy (2003:figs. 55 and 56), and Foord \& DippenaarSchoeman (2005:fig. 3G). However, it matches the illustration by Benoit (1974:fig. 6), based on Tunisian material, and the illustrations by Ribera et al. (1988: figs. 7 and 8, sub $H$. maculata - the confusion of the figures was already pointed out by Peru 2011, but is not yet documented in the WSC). The female genitalia are also closely similar in $H$. sternbergsi (Marusik \& Fet 2009:figs. 7(9) and 8(2)), from Central Asia, but zoogeographic considerations argue against an attribution to this species. H. macullulata, the second species in this genus reported from Spain, has even more coils in the copulatory duct, according to the illustrations in Benoit (1974:fig. 5), Levy (2003:fig. 60), Foord (2008:fig. 32), and Foord \& Dippenaar-Schoeman (2005:fig. 2G).

Tama edwardsi (Lucas, 1846)90b (1+), 104 (1 9 )

\section{Leptonetidae}

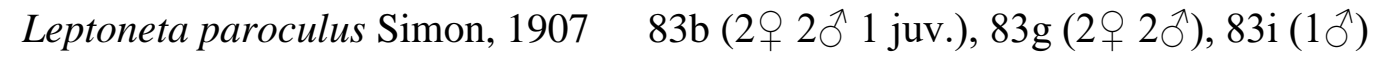

Figure 15 (Breitling 2018:figure 2H)

\section{Linyphiidae}

Acartauchenius scurrilis (O. P.-Cambridge, 1873) 121n (1ठ)

Agyneta cauta (O. P.-Cambridge, 1903) 23 (2ð), 83f(1ð)

Agyneta cf. alpica Tanasevitch, $2000 \quad 16\left(2 \bigcirc^{\Uparrow}\right) \quad$ Figure 16A, B

Agyneta equestris (L. Koch, 1881) 122d (2q 3ठ) NEW FOR SPAIN Figure 16C

Agyneta fuscipalpa (C. L. Koch, 1836) 83c (10̂)

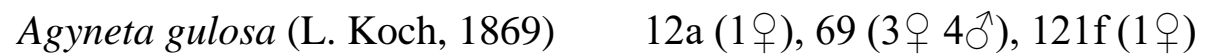

Agyneta nigripes (Simon, 1884) 21c (15), 27a (1), 31 (3q), 121i (2q) NEW FOR

\section{SPAIN}

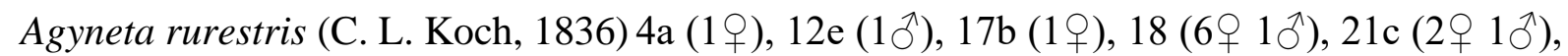

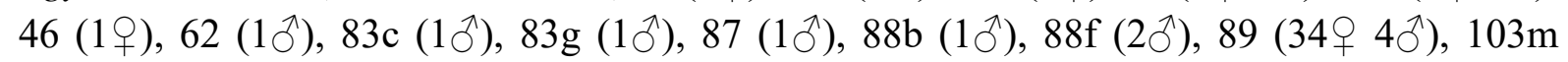

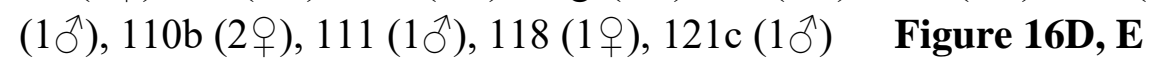

Alioranus pauper (Simon, 1881) 7b (1ठ), 93a $(4 \circ 2 \widehat{\bigcirc})$

Araeoncus anguineus (L. Koch, 1869) 18 (5), 55 (1q), 69 (1q $\left.1 \bigcirc^{\Uparrow}\right)$ NEW FOR FRANCE Figure 17A

Bathyphantes approximatus (O. P.-Cambridge, 1871) 38 (1우)

Bathyphantes gracilis (Blackwall, 1841) 54 (1ㅇ), $86\left(1{ }^{\Uparrow}\right)$ 
Bolyphantes alticeps (Sundevall, 1833) 34 (1ㅇ)

847 Bolyphantes luteolus (Blackwall, 1833) 27b (2), $40 \mathrm{~b}(1+$ ), 41 (1ㅇ)

848 Canariphantes ritae Bosmans, $1985104(1$ ) $\quad$ Figure 17B

849 This species was transferred from Lepthyphantes by Bosmans et al. (2019).

850

\section{Canariphantes tenerrimus (Simon, 1929) $104(1 \odot 1 \overbrace{}^{\Uparrow})$ Figure 16F, 17C}

852 The shape of the lamella characteristica appears to be quite variable in this species. In this 853 male specimen, it resembles the shape illustrated by Denis (1934; sub Lepthyphantes 854 homonymus); the identification is confirmed by the presence of a matching female in the same sample.

856

Canariphantes zonatus (Simon, 1884)

$9\left(1 \circlearrowleft^{\Uparrow}\right)$ Figure 16G

858 Caracladus avicula (L. Koch, 1869) 69 (1우)

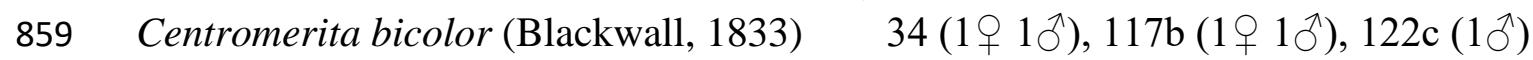

860 Centromerita concinna (Thorell, 1875) 12c (1q), 40b (2q), 46 (1q), 117a (1q)

861 Centromerus albidus Simon, 1929 27a (1q), 103n (8甲) Figure 17D, E

862 Centromerus capucinus (Simon, 1884) 84 (19)

863 Centromerus dilutus (O. P.-Cambridge, 1875) 83g (1ठ), 97d (6ㅇ 3ð)

864 Centromerus isaiai Bosmans, $2015 \quad 47 \mathrm{c}(1+$ ) $\quad$ (Breitling 2018:figure 2N)

865 Centromerus pabulator (O. P.-Cambridge, 1875) $109(1 \circlearrowleft) \quad$ Figure 18A

866 Centromerus prudens (O. P.-Cambridge, 1873) 83g (1ㅇ), 89 (1ㅇ), 96a (3ㅇ)

867 Centromerus satyrus (Simon, 1884) 47c (1q), 47h (2q)

868 Centromerus sellarius (Simon, 1884)83g (1ㅇ)

869 Centromerus semiater (L. Koch, 1879) $38(89)$

870 Centromerus sylvaticus (Blackwall, 1841) 34 (1우 2ð)

871 Ceratinella brevipes (Westring, 1851) 61 (19)

872 Ceratinella brevis (Wider, 1834) 14e (2仓), $33(1+), 111(1 \lesssim)$

873 Ceratinella scabrosa (O. P.-Cambridge, 1871) $36(1$ + $)$

874 Cinetata gradata (Simon, 1881) 116m (14q 2ð)

875 Cnephalocotes obscurus (Blackwall, 1834) 14e (1ठ), 22a (1q)

876 Collinsia inerrans (O. P.-Cambridge, 1885) $69(1 \lesssim$ )

877 Cresmatoneta mutinensis (Canestrini, 1868) 7b (1ठ̂)

878 Dicymbium tibiale (Blackwall, 1836) 14c (19)

879 Diplocephalus arnoi Isaia, $2005 \quad 63 \mathrm{~b}(2$ (2)

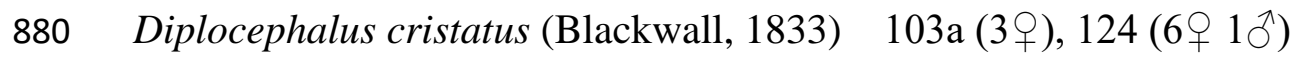

881 Diplocephalus graecus (O. P.-Cambridge, 1873) 54 (1ㅇ)

882 Diplocephalus helleri (L. Koch, 1869) 34 (20ㅇ 6す)

883 Diplocephalus latifrons (O. P.-Cambridge, 1863) 79 (19), 96a (3)

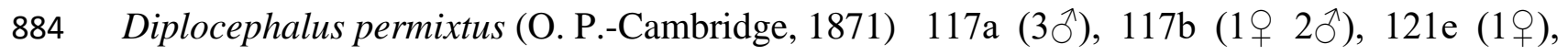
$885121 \mathrm{~h}(1$ q $)$

886 Diplocephalus picinus (Blackwall, 1841) 63a (19), 103b (19)

887 Diplocephalus protuberans (O. P.-Cambridge, 1875) 6 (1ㅇ), $17 \mathrm{~b}$ (1), 55 (5), 1211

888 (6) Figure 17F

889 Diplostyla concolor (Wider, 1834) (Wider, 1834) $50(1 \overbrace{}^{\Uparrow}), 62(1 \overbrace{}^{\Uparrow}), 120 \mathrm{e}(1 \bigcirc)$ 
890 Erigone atra Blackwall, $183314 \mathrm{f}(3 \circ), 18(3 \circ), 52 \mathrm{a}\left(2 \bigcirc^{\Uparrow}\right), 52 \mathrm{c}\left(1+2{ }^{\Uparrow}\right), 123$ (1ㅇ)

891 Erigone cristatopalpus Simon, $188418\left(1{ }^{\Uparrow}\right)$

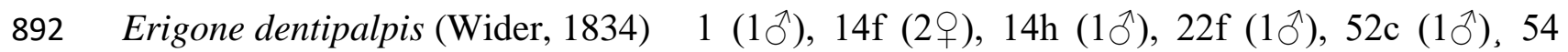

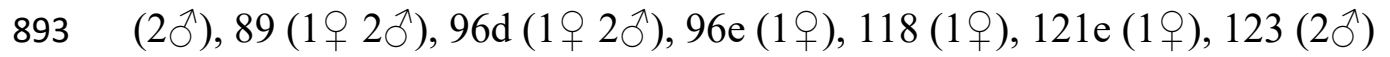

$894 \quad$ Erigone jugorum Simon, $1884 \quad 17 \mathrm{~b}(3$ ㅇ)

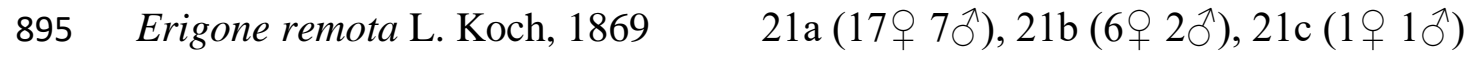

896 Erigone tenuimana Simon, $188418(2$ ㅇ)

897 Erigonella subelevata (L. Koch, 1869) 69 (19)

898 Frontinellina frutetorum (C. L. Koch, 1834) $51\left(1 \bigcirc^{\Uparrow}\right), 88 \mathrm{~g}\left(2 q 1{ }^{\Uparrow}\right), 103 \mathrm{e}(1 \bigcirc), 103 \mathrm{e} 2(1 q$ $\left.8991 \bigcirc^{\Uparrow}\right), 103 \mathrm{f}\left(1 \delta^{\Uparrow}\right), 113 \mathrm{c}(3 \circ), 113 \mathrm{~d}\left(1+1 \delta^{\Uparrow}\right), 115 \mathrm{a}\left(1 \delta^{\Uparrow}\right), 121 \mathrm{k}(1+)$

900 Gnathonarium dentatum (Wider, 1834) $38(11$ + $), 124(1 \precsim)$

901 Gonatium paradoxum (L. Koch, 1869) 103n (1+) NEW FOR SPAIN

902 Gongylidiellum vivum (O. P.-Cambridge, 1875) 102 (1 9 )

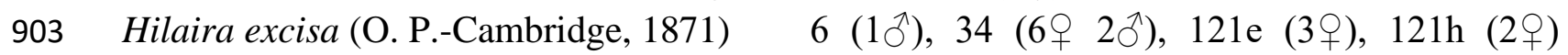

904 NEW FOR SPAIN Figure 18B

905 Hypomma bituberculatum (Wider, 1834) 38 (6ๆ 4ð)

906 Hypomma fulvum (Bösenberg, 1902) 38 (3우 2ð)

907 Improphantes decolor (Westring, 1861) 12c (19)

908 Lasiargus hirsutus (Menge, 1869) 22f $\left(4 \bigcirc 4{ }^{\Uparrow}\right)$

909 Lepthyphantes minutus (Blackwall, 1833) 116f(2)

910 Lepthyphantes notabilis Kulczyński, 1887 116a (19)

911 Leptorhoptrum robustum (Westring, 1851) 34 (9ㅇ 3ठో)

912 Linyphia hortensis Sundevall, 183062 (5ㅇ 2 $\left.\jmath^{\Uparrow}\right)$

913 Lophomma punctatum (Blackwall, 1841)23 (1우)

914 Mansuphantes mansuetus (Thorell, 1875) 41 (1ㅇ)

915 Mansuphantes pseudoarciger (Wunderlich, 1985) 69 (2ð)

916 Mansuphantes simoni (Kulczyński, 1894) 20 (2q), 39c (29)

917 Maso sundevalli (Westring, 1851) 103j (1), 120b (1ㅇ)

918 Mecopisthes nicaeensis (Simon, 1884) $29(1+$ ) $\quad$ Figure 19A

919 This specimen clearly belongs to the same species as the female illustrated by Lecigne (2018; 920 sub Mecopisthes sp., from the Côte d'Azur, less than $100 \mathrm{~km}$ west of the type locality of $M$. 921 nicaeensis in Nice). Duffey's specimen was collected another $150 \mathrm{~km}$ further west along the 922 French coast.

923

924 Mecynargus paetulus (O. P.-Cambridge, 1875)

$18(3 q), 21 \mathrm{a}\left(65 q 27 \delta^{\Uparrow}\right), 21 \mathrm{~b}\left(4 q 2 \delta^{\Uparrow}\right)$

$92521 \mathrm{c}\left(6 \bigcirc 3{ }^{\Uparrow}\right), 55(2 \precsim)$

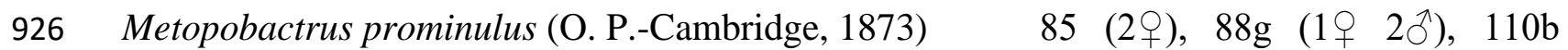

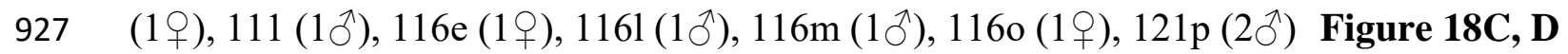

928 The illustrated male belongs to the schenkeli form. Its occurrence together with typical 929 specimens and the identical shape of the genitalia comfirms the synonymy proposed by Muff 930 et al. 2007. This species is remarkably polymorphic in the shape of the male prosoma, as 931 described by Crespo et al. 2018.

932

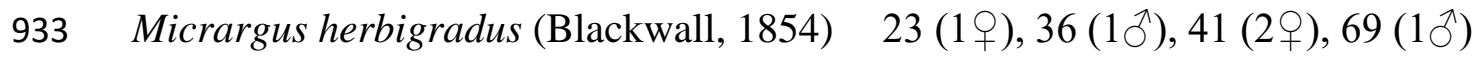


Micrargus laudatus (O. P.-Cambridge, 1881) $1160(1+$ )

935 Microctenonyx subitaneus (O. P.-Cambridge, 1875) 92 (1q), 121i (1 9 )

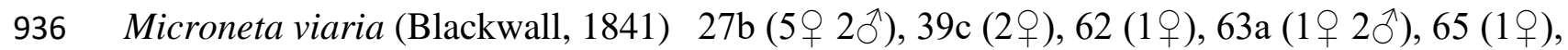

$93783 \mathrm{c}(1$ + $), 83 \mathrm{~g}\left(1 \jmath^{\Uparrow}\right), 96 \mathrm{~b}(1$ + $)$

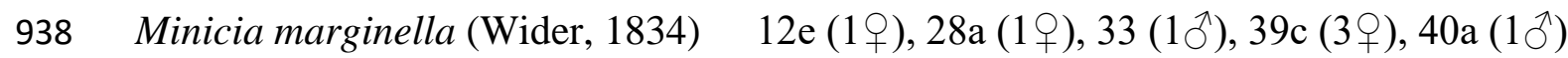

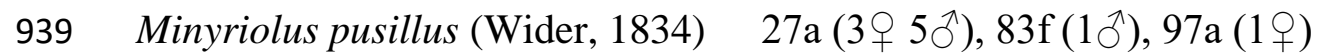

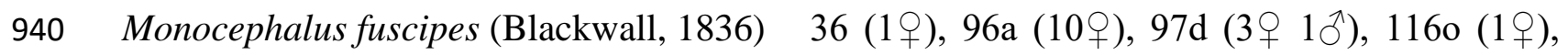
$941122 \mathrm{~b}(2$ ㅇ $), 122 \mathrm{c}\left(1 \circlearrowleft^{\Uparrow}\right)$

942 Neriene furtiva (O. P.-Cambridge, 1871) 7b (1q), 22e (2ㅇ), 22f (1ㅇ), 28a (1ㅇ)

$943 \quad$ Neriene peltata (Wider, 1834) 97b (1)

944 Nusoncus nasutus (Schenkel, 1925) 36 (1ठ), 62 (1ㅇ)

945 Obscuriphantes obscurus (Blackwall, 1841) 116m (1q3ð), 121d (1 9 )

946 Oedothorax agrestis (Blackwall, 1853) 2 (1q), 52c (3), 55 (1), 117b (3)

947 Oedothorax apicatus (Blackwall, 1850) $124\left(1{ }^{\Uparrow}\right)$

948 Oedothorax fuscus (Blackwall, 1834)14f (1), 116k (4 1ð), 117a (1ठ)

949 Oedothorax gibbosus (Blackwall, 1841) $23(4+2 \Im)$

950 Oedothorax retusus (Westring, 1851)13 (1ㅇ), 34 (2ㅇ), 117b (1ㅇ), 121e (2ㅇ), 121h (2q 2§), $951 \quad 121 \mathrm{j}(2$ + $)$

952 Oreonetides glacialis (L. Koch, 1872) 21a $(4 \circ 3$ đ

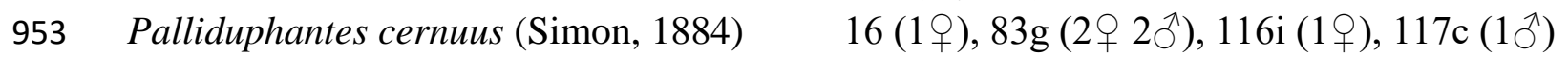

$954 \quad$ Palliduphantes ericaeus (Blackwall, 1853) 23 (3q)

955 Palliduphantes insignis (O. P.-Cambridge, 1913) 22d (19)

956 Palliduphantes pallidus (O. P.-Cambridge, 1871) 39a (1ㅇ)

957 Parapelecopsis nemoralis (Blackwall, 1841)22f (1), 40a (3ㅇ 1ð), 88c (1q), 115b (1우), $958116 \mathrm{i}(1$ ) $)$

959 Pelecopsis bucephala (O. P.-Cambridge, 1875) 93c (25 9 ) Figure 19C

960 Pelecopsis elongata (Wider, 1834) 27a (2q), 47b (1ㅇ), 47d (1ㅇ), 62 (1ㅇ), 70 (1ㅇ)

961 Pelecopsis inedita (O. P.-Cambridge, 1875) 90a (1ठ)

962 Pelecopsis parallela (Wider, 1834) 58b (1ठ), 96e (1ㅇ), 116o (1ठ)

963 Peponocranium ludicrum (O. P.-Cambridge, 1861) 12e (1)

964 Piniphantes pinicola (Simon, 1884) 27a (1q), 121f (1q)

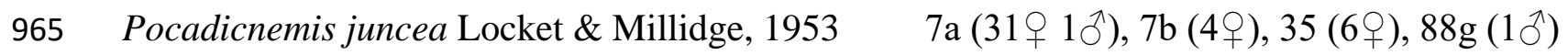

966 Pocadicnemis pumila (Blackwall, 1841) $23\left(4 \circ 2 \bigcirc^{\Uparrow}\right), 39 \mathrm{c}\left(13 \circ 4{ }^{\Uparrow}\right), 52 \mathrm{c}(1$ 우)

967 Porrhomma convexum (Westring, 1851) $34\left(4 \circ 1{ }^{\Uparrow}\right) \quad$ Figure 18F

968 Porrhomma microphthalmum (O. P.-Cambridge, 1871) 54 (1ㅇ)

969 Porrhomma pygmaeum (Blackwall, 1834) $38(1 \lesssim$ )

970 Prinerigone vagans (Audouin, 1826) $18(1+$ ) $, 52 \mathrm{~b}(1 \circlearrowleft), 116 \mathrm{k}(2 \bigcirc)$

$971 \quad$ Saaristoa abnormis (Blackwall, 1841) $83 \mathrm{~b}(1+1 \bigcirc)$

972 Silometopus curtus (Simon, 1881) 7b (19) (Breitling 2018:figure 2O)

973 Sintula furcifer (Simon, 1911) 97e (19)

974 Styloctetor romanus (O. P.-Cambridge, 1873) 22d (1) , 22e (1ㅇ), 40b (1ठ), 96e (1ठ), $975105(1$ ) $)$

976 Tapinocyba mitis (O. P.-Cambridge, 1882) 116i (4)

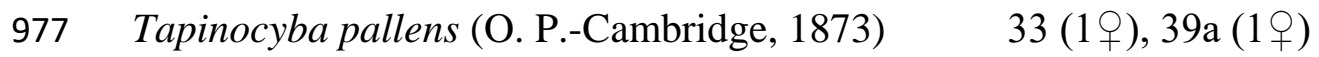


Tapinopa longidens (Wider, 1834) 103j (1), 103k (1+), 120f(1)

Tenuiphantes flavipes (Blackwall, 1854) $\quad 83 \mathrm{f}(2+), 84$ (1), 88a (2), 115b (2), 124 $(1$ )

Tenuiphantes herbicola (Simon, 1884)

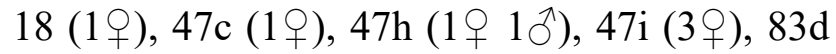
(2ㅇ), $121 \mathrm{j}(1+$ )

Tenuiphantes tenebricola (Wider, 1834) $62(19)$

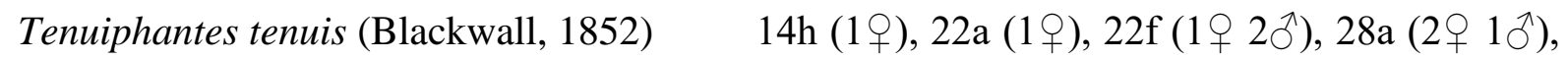

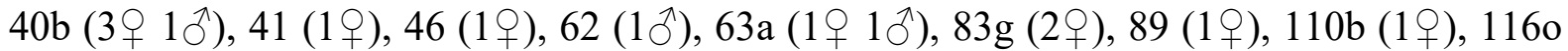
$(2$ 웅

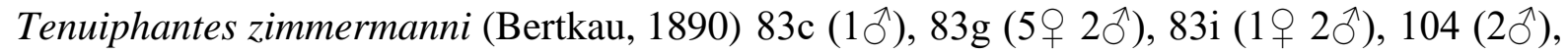
$122 \mathrm{~b}(1+$ )

Theonina cornix (Simon, 1881)

$47 \mathrm{~d}\left(4 \circ 1{ }^{\Uparrow}\right), 47 \mathrm{i}\left(1+1{ }^{\Uparrow}\right)$

Tiso vagans (Blackwall, 1834)

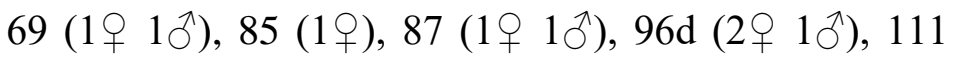
$(1 \precsim), 1160(2 \precsim)$

\section{Trichoncus cf. auritus (L. Koch, 1869) 12c (1), 12e (1+), 40a (1q) Figure 19D}

The epigyne of these specimens differs considerably from that of East European females illustrated, e.g., by Miller (1947, sub Trichoncus kulczynskii), but shows close similarity to that of Swiss specimens illustrated by Wunderlich (2011), who already expressed doubt about the conspecificity of this material. It is, thus, quite possible that Duffey's French specimens represent a vicariant West European sister species of T. auritus s. str. The genus clearly is in need of revision, and this identification, as well as that of the subsequent species, remains somewhat doubtful.

Trichoncus helveticus Denis, 1965 12a (1甲)

Trichoncus saxicola (O. P.-Cambridge, 1861) $87\left(1\right.$ 穴), $89\left(1{ }^{\Uparrow}\right), 103 \mathrm{~s}\left(1{ }^{\Uparrow}\right), 121 \mathrm{k}\left(1{ }^{\Uparrow}\right)$,

\section{1n (1) $\quad$ Figure 18G}

The illustrated male seems to resemble most closely the form described by Denis (1965) as T. varipes, and synonymized by Wunderlich (2011), especially in the shape of the dorsal tibial apophysis.

Trichoncus scrofa Simon, 1884 39c $\left(1 \bigcirc^{\Uparrow}\right)$

Trichoncus vasconicus Denis, 194420 (1우), 120e (1우)

Trichopterna cito (O. P.-Cambridge, 1873) 22e (3ㅇ), 28a (2ㅇ), 40b (1ㅇ), 1160 (1ㅇ) NEW

\section{FOR SPAIN}

Typhochrestus digitatus (O. P.-Cambridge, 1873) 12c (19), 83c (1q)

Walckenaeria acuminata Blackwall, $1833 \quad 83 \mathrm{~g}$ (1ㅇ), 103r(1)

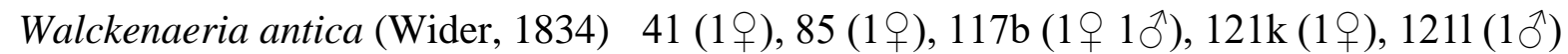

Walckenaeria corniculans (O. P.-Cambridge, 1875) 119 (19)

Walckenaeria cucullata (C. L. Koch, 1836) 116i (1ㅇ)

Walckenaeria dalmasi (Simon, 1914) 83f (3우 1ठ), 83g (1우)

Walckenaeria vigilax (Blackwall, 1853) 21c (2ๆ 2ð)

\section{Liocranidae}

Apostenus fuscus Westring, $1851 \quad 83 \mathrm{~b}(1+), 83 \mathrm{~g}(2 \uparrow), 83 \mathrm{i}(1 \overbrace{}^{\Uparrow}), 103 \mathrm{c}(1+)$ 


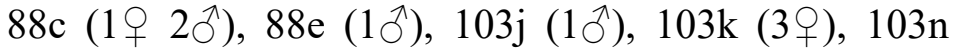
$(1 \circlearrowleft), 113 \mathrm{a}(1+$ ) $), 115 \mathrm{~b}(2$ ㅇ $)$

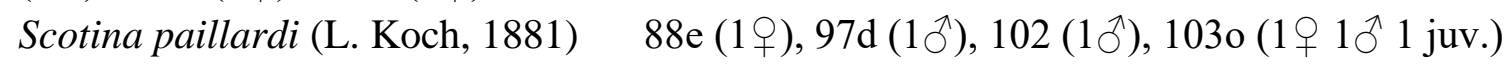

\section{Lycosidae}
Alopecosa albofasciata (Brullé, 1832)
$7 \mathrm{a}(1 \hat{\jmath})$

Alopecosa cuneata (Clerck, 1757) 12c (1ठ), 12d (1q 1ठ), 14a (12ð), 14e, 39b (1ठ), 40b (1ㅇ), 96d (1ठ), 121g (1)

Alopecosa cursor (Hahn, 1831) $12 \mathrm{c}\left(4 \bigcirc^{\Uparrow}\right), 12 \mathrm{~d}(1 \stackrel{+}{)}$

Alopecosa fabrilis (Clerck, 1757) $22 \mathrm{f}(1$ ( $)$

Alopecosa farinosa (Herman, 1879) 12d (1)

Alopecosa pulverulenta (Clerck, 1757) 12c (1), 14a, 14e, 22b (1ठ), 23 (1) , 121k $\left(1 \delta^{\Uparrow}\right)$

Arctosa fulvolineata (Lucas, 1846) $7 \mathrm{~b}(2$ ) $)$

Arctosa lacustris (Simon, 1876) 103d (1甲)

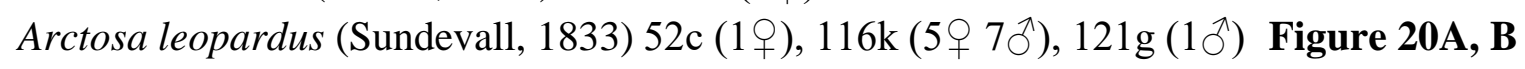
Arctosa maculata (Hahn, 1822) $83 \mathrm{~b}\left(1 \delta^{\Uparrow}\right), 83 \mathrm{c}(1+)$

Arctosa personata (L. Koch, 1872) 22c (1+)

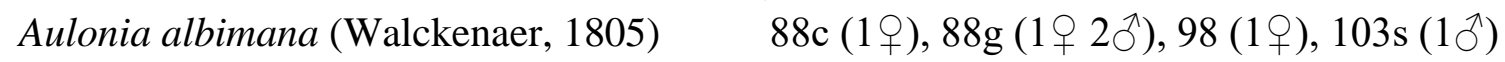

Hygrolycosa rubrofasciata (Ohlert, 1865) $124\left(1 \jmath^{\Uparrow}\right) \quad$ Figure 20C-F

Pardosa agrestis (Westring, 1861) 22f (1ㅇ), $77\left(2 \bigcirc^{\Uparrow}\right)$

Pardosa albatula (Roewer, 1951) $72\left(1 \delta^{\Uparrow}\right) \quad$ (Breitling 2018:figure 2A)

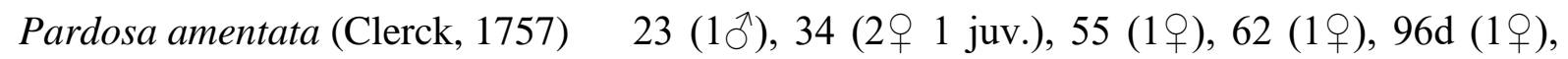
121e $(1 \circ 5 \AA), 121 \mathrm{j}(2 \circ 2 \hat{\bigcirc})$

Pardosa bifasciata (C. L. Koch, 1834) $\quad 88 \mathrm{~g}(2+2 \precsim), 116 \mathrm{p}(2 ð)$

Pardosa cribrata Simon, $1876 \quad 77$ (19)

Pardosa femoralis Simon, 1876 116k (2q 1ठ) Figure 21A

Pardosa lugubris (Walckenaer, 1802) 62 (1우우)

\section{Pardosa mixta (Kulczyński, 1887) 55 (1q) $\quad$ Figure 21B}

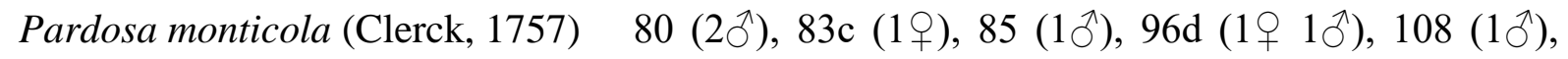

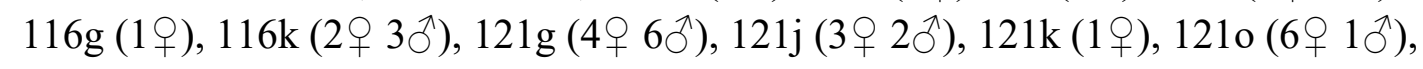

Pardosa morosa (L. Koch, 1870) $83 \mathrm{c}(1+), 84(3+), 95\left(1 \bigcirc^{\Uparrow}\right), 103 \mathrm{~d}\left(5 \uparrow 1 \delta^{\Uparrow}\right) \quad$ Figure

\section{C, 22A}

Pardosa nigriceps (Thorell, 1856) 12c (1ठ), $1160\left(1 \bigcirc 1{ }^{\Uparrow}\right)$

Pardosa oreophila Simon, 1937 17a (1ð), 110b (1우)

Pardosa paludicola (Clerck, 1757) 116k (1ㅇ)

Pardosa prativaga (L. Koch, 1870) 28b (1ð), $44\left(1{ }^{\Uparrow}\right), 52 \mathrm{c}(1$ + $)$

Pardosa pullata (Clerck, 1757) 14a, 14b (1ठ), 14e, 83d (1q), 83g (19), 85 (4ๆ 2ð),

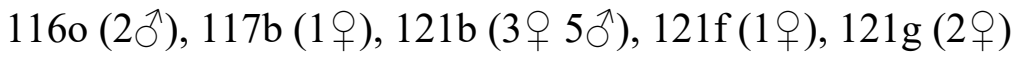

Figure 21D, 22B

The Spanish specimens were reported as P. pyrenaica in Breitling 2018, but a closer examination of more material indicates that they also belong to $P$. pullata, despite the habitat and locality)

Pardosa riparia (C. L. Koch, 1833) $34(1 \overbrace{}^{\Uparrow})$ 


\section{$31(1$ + $) \quad$ Figure 21E}

1069 This specimen, a member of the $P$. monticola group, is likely to belong to the same species as 1070 the specimens reported as Pardosa cf. albatula by Paschetta et al. (2013) and as Pardosa sp. 1071 by Isaia et al. (2015) from alpine pastures in the Italian part of the Alpes Maritimes (M. Isaia, 1072 pers. comm.). The specific identity will remain unclear until a more comprehensive revision 1073 of this difficult species group has been carried out.

1074

Pardosa tenuipes L. Koch, 1882 14a, 14c (1ठ), 14e, 47e (1ठ)

1076 Pardosa wagleri (Hahn, 1822) 103d (1ठ)

1077 Pirata tenuitarsis Simon, $1876 \quad$ 52c (19)

1078 Piratula hygrophila (Thorell, 1872) 52c (2ㅇ), 124 (1ㅇ)

1079 Pyrenecosa pyrenaea (Simon, 1876) 122c (1 9 ) NEW FOR SPAIN

1080 Pyrenecosa rupicola (Dufour, 1821) $89\left(3 \delta^{\Uparrow}\right), 113 \mathrm{e}\left(1 \circlearrowleft^{\Uparrow}\right) \quad$ Figure 22C

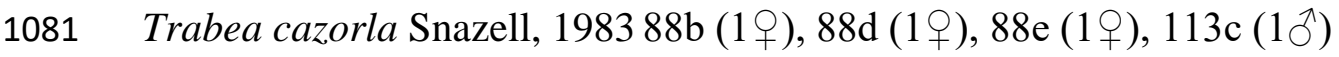

Figure 22D, E

1082 Trochosa ruricola (De Geer, 1778) 52c (2)

1083 Trochosa terricola Thorell, 1856 14e, 116k (1ㅇ), 121k (1ㅇ)

1084 Xerolycosa miniata (C. L. Koch, 1834) 77 (1ठึ)

1085 Xerolycosa nemoralis (Westring, 1861) 45a (1ठ), 83b (2q 1ठ), 108 (1ठ 1 juv.), 110b

1086 (1ふ), $116 \mathrm{~b}(2+2 \AA)$

1087

1088

\section{Mimetidae}

1089 Ero aphana (Walckenaer, 1802) 14i (1 $\quad$ ) $), 107 \mathrm{a}(1$ ㅇ $)$

1090 Mimetus laevigatus (Keyserling, 1863) 113c (1) $\quad$ Figure 23

1091

1092

\section{Miturgidae}

1093 Zora manicata Simon, 1878 12e (1q)

1094 Zora parallela Simon, 1878 22e (2q), 35 (1 juv.), 88g (1 9 2ð)

1095 Zora pardalis Simon, 1878 88c (1), 88g (1)

1096 Zora spinimana (Sundevall, 1833) 41 (1우), 120d (1웅)

1097

1098

1099

\section{Nemesiidae}

1100

1101

Nemesia dorthesi Thorell, 1875

$101(10)$

1102

1103

1104

\section{Oecobiidae}

Uroctea durandi (Latreille, 1809) 103p (1 juv.), 113c (19)

\section{Oonopidae}

1107 Even though the male pedipalp differs considerably from earlier illustrations by Duffey \& 1108 Brignoli (1981:fig. 1), the illustration is based on material from the same sample. The 1109 similarity to Oonops gavarrensis Bosselaers, 2017, is even more pronounced than in the 
1110

1111

1112

1113

1114

1115

1116

1117

1118

1119

1120

1121

1122

1123

1124

1125

1126

1127

1128

1129

1130

1131

1132

1133

1134

1135

1136

1137

1138

1139

1140

1141

1142

1143

1144

1145

1146

1147

1148

1149

1150

1151

1152

1153

earlier illustrations, but this is certainly a different species, especially as the female genitalia are completely different.

Silhouettella loricatula (Roewer, 1942) $\quad$ 47d (1ㅇ)

\section{Oxyopidae}

Oxyopes heterophthalmus (Latreille, 1804) 59 (4우)

\section{Palpimanidae}

Palpimanus gibbulus Dufour, 1820 90b (1)

\section{Philodromidae}

Celerrimus duffeyi Lecigne, Cornic, Oger \& Van Keer, $2019 \quad$ 91a (1ठ) $\quad$ Figure

25A (Breitling 2018:figure 2K)

This highly unusual philodromid was only recently described by Lecigne et al. (2019), with Duffey's material being designated as one of the paratypes.

Philodromus albidus Kulczyński, $1911 \quad$ 14g (1仓), 14i (19)

Philodromus aureolus (Clerck, 1757)76 (2q 1ठ), 121b (3ㅇ)

Philodromus cespitum (Walckenaer, 1802) 141 (1q), 103e (1+)

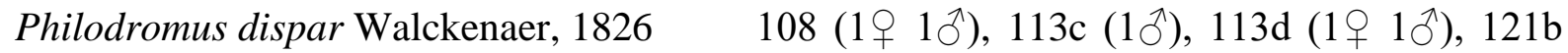

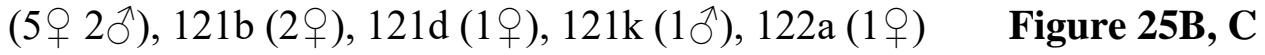

Philodromus emarginatus (Schrank, 1803) 91a (1) , 108 (1ठ), 113d (1ठ), 121b (1ठ) Figure 25D

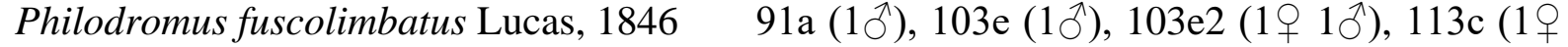
$1 \circlearrowleft), 115 \mathrm{a}(1 \bigcirc 1 \circlearrowleft), 121 \mathrm{k}(2 \bigcirc 1 \circlearrowleft) \quad$ Figure 25E

Philodromus parietalis Simon, $1875116 \mathrm{f}(1+)$

Philodromus praedatus O. P.-Cambridge, 1871 113e (2今)

Philodromus rufus Walckenaer, $182614 \mathrm{~b}(1 \bigcirc), 54(1 \uparrow), 115 \mathrm{a}(2$ )

Philodromus vagulus Simon, 1875125 (1ㅇ)

Pulchellodromus pulchellus (Lucas, 1846) 22c (2早 1ठ), 22e (2ð), 28a (1千), 115a (1ㅇ)

Thanatus atratus Simon, $1875 \quad 61(1 \precsim)$

Thanatus formicinus (Clerck, 1757) 90b (1+), $104(1+), 107 \mathrm{a}(1+)$

Thanatus cf. lineatipes Simon, 187094 (19) $\quad$ Figure 25F

This specimen is assigned to this poorly described species with some hesitation, based on the photographs of Spanish specimens from Robert Bosmans' collection on the website of Pierre Oger.

Tibellus oblongus (Walckenaer, 1802)

52a $(1 \circ), 103 \mathrm{f}(1 \circ) \quad$ Figure 25G

\section{Pholcidae}

Holocnemus caudatus (Dufour, 1820)

81 (1ㅇ 1 juv.), 92 (1 juv.), 107a (2 juv.), 107b (3ㅇ 2ð), 113d (1ㅇ) Figure 26A-D

Holocnemus hispanicus Wiehle, 1933

90b $\left(1 q 1{ }^{\Uparrow}\right)$ Figure 26E, $\mathbf{F}$ 
1154

1155

1156

1157

1158

1159

1160

1161

1162

1163

1164

1165

1166

1167

1168

1169

1170

1171

1172

1173

1174

1175

1176

1177

1178

1179

1180

1181

1182

1183

1184

1185

1186

1187

1188

1189

1190

1191

1192

1193

1194

1195

1196

1197
Pholcus opilionoides (Schrank, 1781)

26G

Spermophorides mammata (Senglet, 1973) 90a (1+)

\section{Phrurolithidae}

Liophrurillus flavitarsis (Lucas, 1846)

$7 \mathrm{a}(2 \bigcirc 1$ juv.)

Phrurolithus festivus (C. L. Koch, 1835)

84 (1우), 113b (1우 1ठ), 121j (1ठ), $121 \mathrm{o}(1+$ )

Figure 27A, B

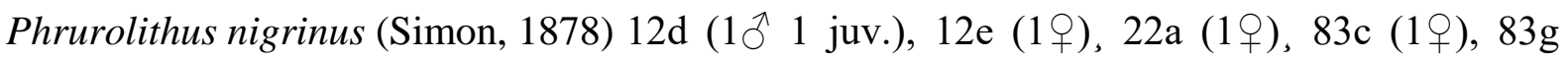
(1q), 84 (2), 113d (19)

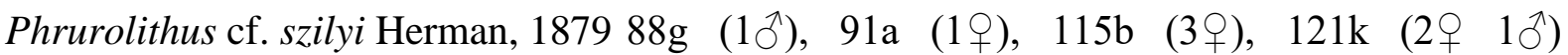
Figure 27C (Breitling 2018:figure 2I)

The identity of these specimens remains slightly dubious. $P$. szilyi shows a strikingly disjunct distribution, being reported on the one hand from the Iberian Peninsula, and on the other hand from Austria and the Czech Republic eastwards. While the male pedipalp appears very similar (see figure in Breitling 2018), the structure of the internal female genitalia in the Spanish specimens is more difficult to reconcile with published illustrations (e.g. Grimm 1986:fig. 55, Komnenov et al. 2016:figs. 83 and 84, and Wiehle 1967:fig. 71). Could this be another case of vicariant sister species?

\section{Pisauridae}

Pisaura mirabilis (Clerck, 1757) 103s (1ठ), 121b (1q) Figure 28

\section{Salticidae}

Aelurillus luctuosus (Lucas, 1846) 81 (1仓), 104 (1ठ)

Aelurillus v-insignitus (Clerck, 1757)40b (3q 3ð), 116g (4ð) $\quad$ Figure 29A,B

Attulus distinguendus (Simon, 1868) 22f (1+)

Ballus chalybeius (Walckenaer, 1802) 14g (1q), $141(1+), 108(3 \%)$

Calositticus rupicola (C. L. Koch, 1837) 34 (1థ1ð), 103m (1ठ)

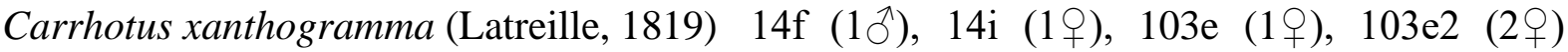
Figure 31A

Chalcoscirtus infimus (Simon, 1868) $29\left(1{ }^{\circledR}\right)$

Cyrba algerina (Lucas, 1846) 107b (1ठ)

Dendryphantes rudis (Sundevall, 1833) 122a (1+)

Euophrys frontalis (Walckenaer, 1802) 22a (2ð), 47d (1ㅇ), 48 (1) $), 83 \mathrm{c}$ (1q), 88b $\left(1+\right.$ ) $, 88 \mathrm{~g}(1 \bigcirc), 108\left(1 \bigcirc 1{ }^{\Uparrow}\right), 110 \mathrm{~b}(1 \circlearrowleft)$

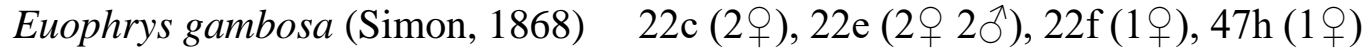

Euophrys rufibarbis (Simon, 1868) 8 (19)

Euophrys sulphurea (L. Koch, 1867) 29 (3ठึ)

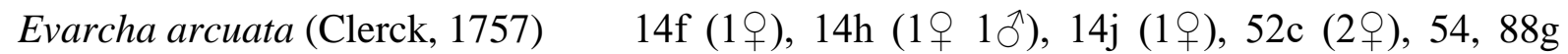
(1ठ), 103r (1우)

Evarcha falcata (Clerck, 1757)

83h $\left(1+10^{\Uparrow}\right), 121 \mathrm{~b}(2 \circ), 121 \mathrm{~d}(1+)$ Figure 31B

Evarcha jucunda (Lucas, 1846)

(Lucas, 1846) $57\left(1{ }^{\Im}\right), 115 \mathrm{~b}(1 \bigcirc)$

Evarcha laetabunda (C. L. Koch, 1846) $\quad 45 \mathrm{c}\left(1 \bigcirc^{\Uparrow}\right), 88 \mathrm{~g}\left(2 \bigcirc^{\Uparrow}\right)$ 
Habrocestum cf. ibericum Dalmas, 1920

The genus Habrocestum in the western Mediterranean is in urgent need of a modern revision. Most species are poorly known and rarely reported, several of them apparently being known only from the male type specimens ( $H$. algericum, $H$. ibericum, $H$. lepidum); all of these might be junior synonyms of a very variable $H$. bovaei (Lucas, 1846), perhaps together with $H$. pullatum (known only from the female), $H$. simoni and $H$. ornaticeps. This synonymy seems to be implicit in Lecigne's (2012) report of $H$. bovaei from Malaga, referring to a specimen with a distinct prosomal pattern and clearly annulated legs, both in direct contradiction of the redescription of H. bovaei in Dalmas's (1920) revision of the genus, but in good agreement with, e.g., H. ornaticeps or $H$. lepidum. The present specimen is tentatively assigned to $H$. ibericum on the basis of the relative closeness to the type locality (Cartagena, on the south coast of Spain, less than $400 \mathrm{~km}$ east of Nerja), the illustrations of the pedipalp of the type provided by Prószyński (1987:fig. 29), mixed white and red hairs around the anterior eyes, and the extension of the white thoracic spot into two parallel bands up to the anterior median eyes ("tache thoracique prolongée jusqu'aux yeux médians par deux bandes blanches parallèles", Dalmas 1920:62). A similarly good match might be provided by $H$. algericum, but this species seems to be considerably darker, according to the original description.

\section{Heliophanus aeneus (Hahn, 1832) $108(1$ † $), 121 \mathrm{f}\left(1 \delta^{\Uparrow}\right)$}

Heliophanus apiatus Simon, 1868 107a $\left(1{ }^{\Uparrow}\right)$

Heliophanus cupreus (Walckenaer, 1802) 72 (2ㅇ), 84 (1ふ), 96c (1ㅇ), 108 (1ふ), 113a

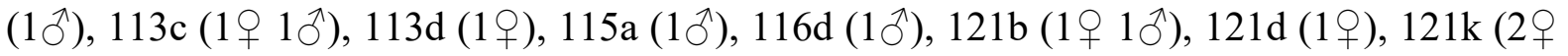
$\left.2{ }^{\Uparrow}\right), 122 \mathrm{a}(1+$ ) $\quad$ Figure 29D, E, 31C-E

Heliophanus dubius C. L. Koch, $1835 \quad 116 \mathrm{f}(1+$ ㅇ), 116m (1ㅇ), 124 (1q)

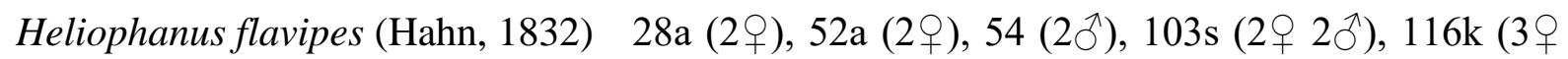
1ठ), $1160\left(1{ }^{\Uparrow}\right)$

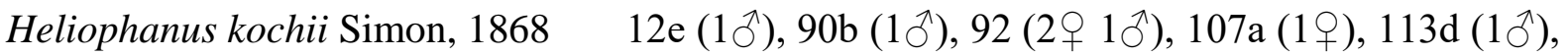
$121 \mathrm{k}\left(1{ }^{\Uparrow}\right)$

Heliophanus lineiventris Simon, $1868 \quad 64$ (1우

Heliophanus melinus L. Koch, 186729 (2ð 1 juv.), 59 (1ふึ)

Heliophanus patagiatus Thorell, $1875 \quad 95(1+6 \hat{\jmath}), 103 \mathrm{~h}(1+) \quad$ Figure 29F

Heliophanus tribulosus Simon, 1868 22c $\left(1 \uparrow 1 \delta^{\Uparrow}\right), 121 \mathrm{k}\left(2 \uparrow 1 \delta^{\Uparrow}\right)$ Figure 29G, 31F

Hypositticus pubescens (Fabricius, 1775) 89 (1へ̂)

Iberattus semiglabratus (Simon, 1868)

89 (1ㅇ), 103n (1아), 103r (2ㅇ), 122a (1ふ), 122b

\section{$\left(1{ }^{\Uparrow}\right) \quad$ Figure 29H}

Icius hamatus (C. L. Koch, 1846) $\quad$ 103e2 (2q), 103f(1ð), 113c (3q) Figure 30A, 32A

The broad retrolateral tibial apophysis could suggest that the male specimen belongs to Icius crassipes; however, the long embolus, the locality in the very North of Spain, and the fact that unambiguous I. hamatus females were collected at the same site on the same day support the identification proposed here.

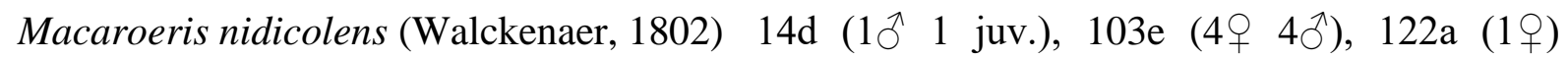
Figure 30B, 32B 
Marpissa muscosa (Clerck, 1757) 47j (1 juv.)

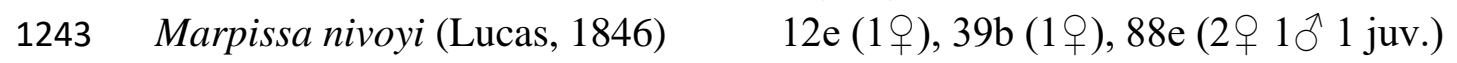

1244 Menemerus semilimbatus (Hahn, 1829) 73 (19)

$1245 \quad$ Neon rayi (Simon, 1875) 88c (2q), 88d (1)

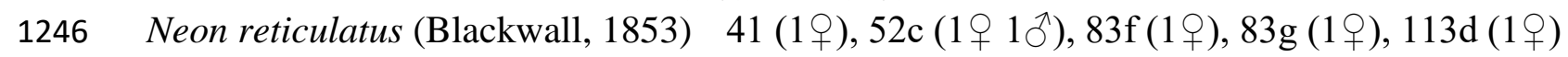

1247 Neon valentulus Falconer, $19127 \mathrm{~b}\left(2{ }^{\Uparrow}\right)$

1248 Pellenes geniculatus (Simon, 1868) $95\left(191{ }^{\Uparrow}\right), 107 \mathrm{~b}\left(1 \delta^{\Uparrow}\right)$

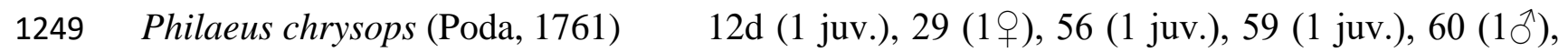

$125071\left(1{ }^{\Uparrow}\right), 113 \mathrm{e}\left(1{ }^{\Uparrow}\right)$

1251 Phlegra bresnieri (Lucas, 1846) 29 (2ᄋ 3ð 1 juv.), 81 (1 9 )

1252 Phlegrafasciata (Hahn, 1826) 12c (1), $121 \mathrm{k}\left(191{ }^{\Uparrow}\right)$

1253 Pseudeuophrys erratica (Walckenaer, 1826) 83g (1ठ), 116m (1థ) Figure 32C

$1254 \quad$ Pseudeuophrys lanigera (Simon, 1871) 12c (1q 1§), $89(1+2 \AA), 116 \mathrm{~g}(2 q)$ Figure

1255 32D

$1256 \quad$ Pseudeuophrys obsoleta (Simon, 1868) $62(19)$

1257 Pseudomogus univittatus (Simon, 1871) 28b (1ठ)

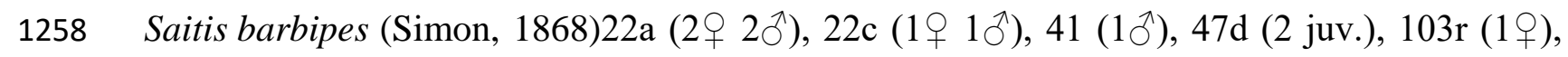
$1259120 \mathrm{f}(1$ ) $)$

1260 Salticus confusus Lucas, 1846 91a (1q), 103e (1ㅇ), 103e2 (1ठ), 108 (1ㅇ), 121j (2ð),

1261 121k $(3 \bigcirc 2 \widehat{\jmath})$ Figure 30C, 32E

1262 Salticus scenicus (Clerck, 1757) 2(1ठ), 39b (1ठ), 121k (1ठ゙)

1263 Synageles hilarulus (C. L. Koch, 1846) 12e (2ð)

1264 Talavera aequipes (O. P.-Cambridge, 1871) 122b (19)

1265 Talavera petrensis (C. L. Koch, 1837) 12c (1ठ), $16(1 q), 40 \mathrm{~b}\left(3 q 5{ }^{\Uparrow}\right), 83 \mathrm{c}(1 q), 121 \mathrm{j}$

$1266(1 \bigcirc 1 \precsim), 1210(2 q)$

1267

1268 Scytodidae

1269 Scytodes thoracica (Latreille, 1802) 47d (1q 1 juv.), 47h (1 + )

1270

\section{Segestriidae}

1273

Segestria senoculata (Linnaeus, 1758)

$12 \mathrm{c}\left(1 \bigcirc 1{ }^{\Uparrow}\right)$

\section{Sparassidae}

\section{Sicariidae}

Loxosceles rufescens (Dufour, 1820) 90b (19), 92 (1) formosa is the only one of the five European species in this genus not previously reported from Spain. Unfortunately, the available figures for the females of several of the known species do not allow a confident discrimination from $M$. formosa, given the major ontogenetic variation of epigynal structures, and the genus appears in need of a thorough revision. 
Micrommata ligurina (C. L. Koch, 1845)

Micrommata virescens (Clerck, 1757)

$\left.7 b(1)^{\Uparrow}\right)$

121d $(2 \widehat{O}), 121 \mathrm{k}(1 \precsim)$ Figure 33B, C

\section{Synaphridae \\ Cepheia longiseta (Simon, 1881) $\quad 47 \mathrm{~h}\left(1{ }^{\Uparrow}\right)$}

\section{Tetragnatidae}

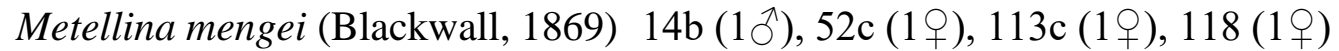

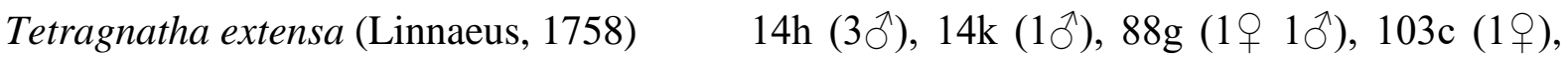
116k $\left(1 \uparrow 1{ }^{\Uparrow}\right)$ Figure 34A-D

The cleared chelicerae of both males and females reveal what appears are derivatives of the venom gland extending into the cheliceral teeth and ending at (openings on?) their tips. Could these be involved in gustatory courtship or the delivery of the sex-specific venom components identified by Binford et al. (2016) and Zobel-Thropp (2018) - or are they just artefacts of the preparation?

\section{Tetragnatha intermedia Kulczyński, $1891114\left(1 \subsetneq 1{ }^{\Uparrow}\right) \quad$ Figure 34E}

Tetragnatha montana Simon, $18742\left(1{ }^{\Uparrow}\right), 14 \mathrm{~d}\left(1 \delta^{\Uparrow}\right), 14 \mathrm{~g}(2 \precsim)$

Tetragnatha obtusa C. L. Koch, $1837 \quad 14 \mathrm{~b}(1$ ㅇ $)$

\section{Theridiidae}

\section{Achaeridion conigerum (Simon, 1914) $84(1 \overbrace{}^{\Uparrow})$ NEW FOR SPAIN}

Anelosimus vittatus (C. L. Koch, 1836) 14g (1) $)$ 14h (1), 14i (4), $141\left(8 \bigcirc 3{ }^{\Uparrow}\right)$

Asagena phalerata (Panzer, 1801) 29 (1ठ), 31 (2ᄋ 1 juv.), 41 (2 juv.), $58 \mathrm{~b}(1$ 우), 89 (1ठ), 96c (1우), 121g (2ㅇ), $121 \mathrm{j}\left(1+1 \delta^{\Uparrow}\right)$

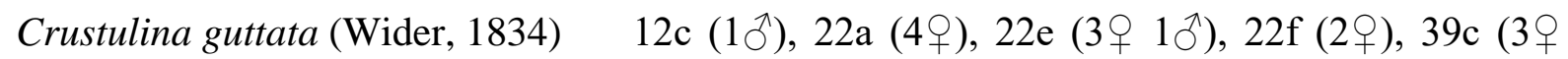

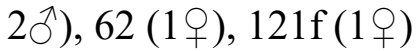

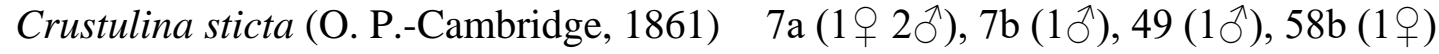

Dipoena braccata (C. L. Koch, 1841) 103e2 (1今)

Dipoena melanogaster (C. L. Koch, 1837) 113c (3우 1〕)

Enoplognatha afrodite Hippa \& Oksala, $1983 \quad$ 120a $(1 \overbrace{}^{\Uparrow}) \quad$ Figure $\quad$ 35A-C NEW FOR SPAIN

The pedipalps of the single male specimen are expanded and consequently difficult to match to the illustrations of E. afrodite in the literature. However, the details that are visible, in combination with the chelicerae and the habitus (which is similar to the lineata form of $E$. ovata), seem to be sufficiently diagnostic.

Enoplognatha diversa (Blackwall, 1859) 107a (1ㅇ)

Enoplognatha franzi Wunderlich, $1995 \quad 81 \quad\left(2+1{ }^{\Uparrow}\right), \quad 107 \mathrm{a} \quad\left(1+1{ }^{\Uparrow}\right), \quad 107 \mathrm{~b} \quad(1+)$ Figure 35D

Enoplognatha oelandica (Thorell, 1875) $22 \mathrm{f}(2$ ) $)$

Enoplognatha testacea Simon, 1884 47d (1ㅇ), 56 (2ㅇ)

Enoplognatha thoracica (Hahn, 1833) 22a (1 9 ), 22c (2q), 22f(1q), 121k (1 9$)$

Episinus truncatus Latreille, 1809 22e (2 juv.), 54 
1333 Euryopis quinqueguttata Thorell, $1875 \quad 22 \mathrm{c}(19)$

1334 Kochiura aulica (C. L. Koch, 1838) 28a (1ㅇ), $30(1 \overbrace{}^{\Uparrow}), 103 \mathrm{f}(1 \overbrace{}^{\Uparrow})$,

1335 Lasaeola minutissima Wunderlich, $2011 \quad 113 \mathrm{c}(1 ठ)$

1336 Lasaeola tristis (Hahn, 1833) 91e (1ふ)

1337 Neottiura bimaculata (Linnaeus, 1767) 22e (5甲), $54(1$ + $), 113 \mathrm{a}\left(2 q 1{ }^{\jmath}\right)$

1338 Neottiura suaveolens (Simon, 1880) 22e (2), 104 (2 juv.)

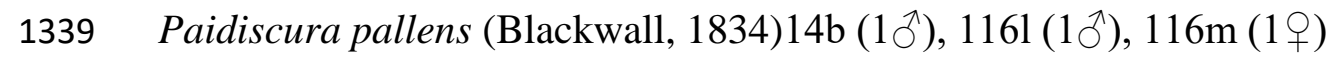

1340 Parasteatoda lunata (Clerck, 1757) 2 (2甲), 14h (1ठ)

1341 Parasteatoda tepidariorum (C. L. Koch, 1841) 10 (1ð 2 juv.)

1342 Pholcomma gibbum (Westring, 1851) 97d (1\%)

1343 Phycosoma inornatum (O. P.-Cambridge, 1861) 12d (2早 3ð)

1344 Phylloneta impressa (L. Koch, 1881)89 (1ㅇ), 103e (1ð̋ 1 juv.), 103m (1

1345 Phylloneta sisyphia (Clerck, 1757) $33(1+), 121 \mathrm{~b}\left(1 \propto 3 \bigcirc^{\Uparrow}\right) \quad$ Figure 35F

1346 Platnickina nigropunctata (Lucas, 1846) 115a (1q)

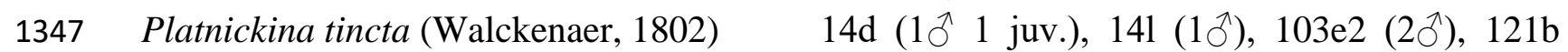

$1348 \quad(1$ ) $)$

1349 Robertus arundineti (O. P.-Cambridge, 1871) 120c (1 9 )

1350 Robertus lividus (Blackwall, 1836) 34 (3), 69 (3)

1351 Robertus mediterraneus Eskov, $198783 \mathrm{c}(1+), 83 \mathrm{~g}(5 q), 122 \mathrm{~b}(3 q) \quad$ Figure 36A

1352 Ruborridion musivum (Simon, 1873) 113c (1ㅇ)

1353 Sardinidion blackwalli (O. P.-Cambridge, 1871) $32(1 \circlearrowleft), 113 \mathrm{c}(1 \circlearrowleft)$

1354 Simitidion simile (C. L. Koch, 1836) 52a (10)

1355 Steatoda albomaculata (De Geer, 1778) 40b (1థ1ð 1 juv.), 46 (1 juv.), 73 (2ᄋ)

1356 Steatoda grossa (C. L. Koch, 1838) 90b (1థ), 107a (1ठ 1 juv.)

1357 Steatoda paykulliana (Walckenaer, 1806) 81 (1甲), $86(1$ + $), 106(1$ + $), 107 \mathrm{a}(1$ q $)$

1358 Theonoe minutissima (O. P.-Cambridge, 1879) 52c (1우 1ठ)

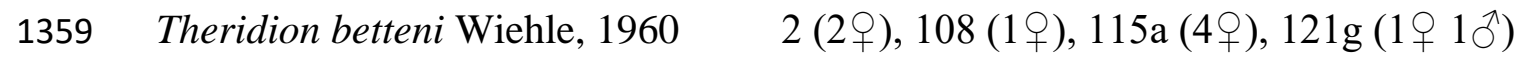

1360 Figure 35G, H, 36B (Breitling 2018:figure 2B) NEW FOR SPAIN

1361 Theridion harmsi Wunderlich, 2011 113c (2ᄋ 1ठ)

1362 Theridion hannoniae Denis, 1945 90a $(1 \circlearrowleft), 107 \mathrm{~b}\left(1 \delta^{\Uparrow}\right)$ Figure 35I

1363 Theridion melanostictum O. P.-Cambridge, $1876 \quad 86(1$ + $)$

1364 Theridion melanurum Hahn, $18313\left(1{ }^{\Uparrow}\right), 45 \mathrm{~b}(1$ )

1365 Theridion mystaceum L. Koch, $187022 \mathrm{~d}(1 \circ), 116 \mathrm{f}(1 \overbrace{}^{\Uparrow}), 121 \mathrm{~m}(1 \overbrace{}^{\Uparrow}) \quad$ Figure 35J

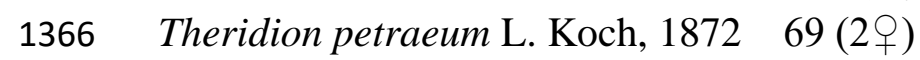

1367 Theridion pictum (Walckenaer, 1802) $124(1+)$

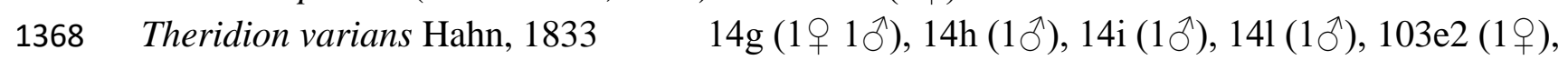

$1369121 \mathrm{~b}\left(1 \jmath^{\Uparrow}\right), 122 \mathrm{~b}\left(2 \delta^{\Uparrow}\right)$

1370 Theridion wiehlei Schenkel, $1938 \quad 107 \mathrm{~b}(2$ q) $\quad$ Figure 36C

1371 Identification of these specimens is based on comparison to the illustrations provided by 1372 Wiehle (1937) and drawings of a female collected by Simon in Algeria (provided by B. 1373 Thaler-Knoflach, Figure 36D). The internal genitalia of these females appear to be very 
similar to those described for Theridion negebense Levy \& Amitai, 1982. Levy \& Amitai (1982) seem to have been unaware of the existence of $T$. wiehlei when they diagnosed the new species as separated "easily from all other Theridion species"; it is thus quite possible that $T$. negebense will turn out to be a junior synonym of $T$. wiehlei. The record of $T$. negebense from Cadiz, Spain, reported by Vanuytven et al. (1994), and the one from Almería mentioned by Barrientos et al. (2017), are both based on single females and with high probability refer to $T$. wiehlei, which was originally described from southern Spain. $T$. negebense thus should be deleted from the European species list. The male of T. wiehlei is not known with certainty: when Schenkel described the species, he apparently only had a single female specimen available (the holotype illustrated by Deltshev 1992). For the male, Schenkel referred to Wiehle (1937), who together with a female of T. wiehlei had illustrated a male collected by L. Koch in Eltersdorf, Germany, both as Theridion petraeum. However, while Koch (1877) reports collecting mature males and females, Wiehle's text makes it clear that the part of Koch's material available to him contained mature males only. It is not clear where the female of $T$. wiehlei, which Wiehle illustrated as T. petraeum, came from, as he later states that he only had Koch's material at this point (Wiehle 1960b), but it is interesting that in May 1930 Wiehle had been on a collection trip around Granada, quite close to the type locality of $T$. wiehlei. There is currently no reason to assume that the male and female illustrated by Wiehle are conspecific, and later reports of male T. wiehlei (e.g., by Denis 1945) also refer to specimens collected without accompanying females. In fact, on zoogeographic grounds, it seems much more likely that the Eltersdorf male illustrated by Wiehle, as well as the males illustrated by Denis (1945) from France and by Tyshchenko (1971) from Russia, actually refer to $T$. cinereum, the palp of which matches that illustrated by Wiehle so closely that specimens from the Alps were originally misidentified as T. wiehlei (Thaler 1981). True T. wiehlei seems, therefore, to be restricted to the very South of Spain and North Africa, possibly extending east to Israel.

\section{Thomisidae}

Cozyptila blackwalli (Simon, 1875) 47c (2ㅇ 1 juv.), 122d (1 $\odot)$

Diaea dorsata (Fabricius, 1777) 116j (1ㅇ), 121b (1+)

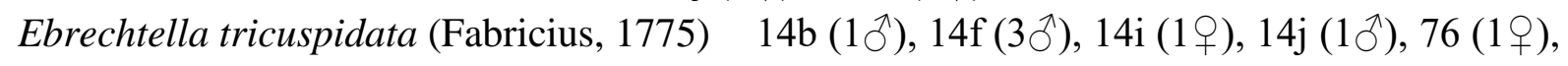
$78\left(1{ }^{\Uparrow}\right), 141\left(2{ }^{\Uparrow}\right)$

\section{Heriaeus oblongus Simon, 1918 122a $(1 \overbrace{}^{\Uparrow})$}

\section{Heriaeus simoni Kulczyński, 190359 (1今̂) (Breitling 2018:figure 2M)}

Misumena vatia (Clerck, 1757) 14f (2ð), 14h (1仓), 14i (1q), 71 (1q), 78 (1q), 83h $(1+), 108\left(2 \bigcirc^{\Uparrow}\right), 121 \mathrm{~b}(2 \circ), 121 \mathrm{k}(1+) \quad$ Figure 37A, B

Monaeses paradoxus (Lucas, 1846) 107a (1+)

Ozyptila atomaria (Panzer, 1801) 40b (1), 96c (1ๆ 2ð)

Ozyptila confluens (C. L. Koch, 1845) $66(1 \lesssim$ त)

Ozyptila furcula L. Koch, $1882 \quad 7 \mathrm{a}\left(1 \bigcirc^{\Uparrow}\right), 7 \mathrm{~b}\left(1 \delta^{\Uparrow}\right)$

Ozyptila rauda Simon, 187569 (2q 1ठ)

Pistius truncatus (Pallas, 1772) 72 (1ठ), 103i (2 juv.)

Runcinia grammica (C. L. Koch, 1837) 57 (1ठ), $61(1 ふ)$ 
1417

1418

1419

1420

1421

1422

1423

1424

1425

1426

1427

1428

1429

1430

1431

1432

1433

1434

1435

1436

1437

1438

1439

1440

1441

1442

1443

1444

1445

1446

1447

1448

1449

1450

1451

1452

1453

1454

1455

1456

1457

1458

1459

1460

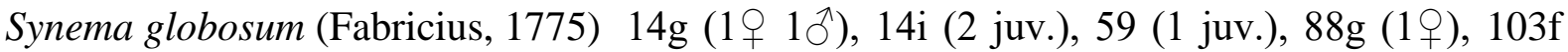
(2ㅇ 1ठ), 115a (1ㅇ 4 juv.), 122a (1ठ)

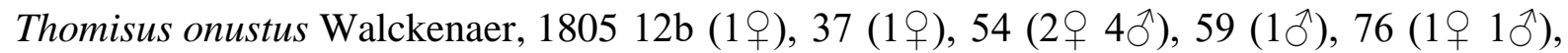
115a $(1 \precsim) \quad$ Figure 37C

Tmarus punctatissimus (Simon, 1870) $\quad$ 103f $(1 \circlearrowleft) \quad$ Figure 37D, E

Tmarus stellio Simon, 1875 116c (1ㅇ 1ð) Figure 8E, 37F, G

Xysticus audax (Schrank, 1803) 94 (1ठ), 103e2 (1ठ), 108 (3ठ)

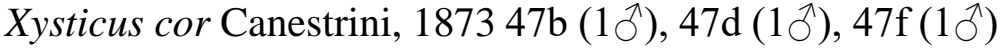

Xysticus cristatus (Clerck, 1757) $\quad 40 \mathrm{a}\left(1 \bigcirc^{\Uparrow}\right), 54(4), 83 \mathrm{c}(1+$ )

Xysticus desidiosus Simon, $187531(2 \circ)$

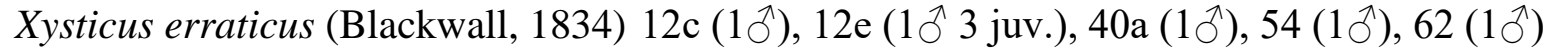

Xysticus gallicus Simon, $1875 \quad 62$ (1우시), 80 (1우)

Xysticus ibex Simon, $187598(1 \bigcirc) \quad$ (Breitling 2018:figure 2P)

Xysticus kempeleni Thorell, 1872 14c (1ठ), 103e2(1 9$), 121 \mathrm{k}\left(1{ }^{\Uparrow}\right)$

Figure 37H

Xysticus kochi Thorell, 1872 14h (1ㅇ), 29 (1ठ), 40a (1ठ), 77 (1ठ)

Xysticus lanio C. L. Koch, $1835 \quad 97 \mathrm{~d}(1+), 121 \mathrm{~b}(1+), 121 \mathrm{~d}\left(1{ }^{\Uparrow}\right)$

Xysticus macedonicus Šilhavý, $194472(1 \bigcirc)$

(Breitling 2018:figure 2E)

Xysticus ninnii Thorell, 1872 22c (1ㅇ), 43 (1ठ)

Xysticus nubilus Simon, $1875101(3 ð) \quad$ (Breitling 2018:figure 2Q)

Xysticus thessalicoides Wunderlich, 199561 (19)

Xysticus cf. fienae (Jocqué, 1993) 107a (1 1 )

This could also be $X$. tristrami (O. P.-Cambridge, 1872), the known published distribution of which is restricted to the eastern Mediterranean, while $X$. fienae was originally described from the south coast of Spain. Based on the descriptions and few illustrations in the literature (e.g. Jocqué 1993:fig. 1-3, Kiany et al. 2017:fig. 14, Kulczyński 1911: figs. 31, 34 and 35, Lecigne 2016:figs. 11E and F, Levy 1976:figs. 9-12, and Marusik \& Logunov 1995:figs. 5254), I am unable to confidently distinguish the two species. The decision whether we are dealing with two vicariant sister species or with a single widespread species will require examination of a larger amount of material.

Xysticus ulmi (Hahn, 1831) 14e (1ठึ), 14g (1ㅇ), 14h (2ᄋ)

\section{Titaenoecidae}

Titanoeca monticola (Simon, 1870) 113a $(2 \overbrace{}^{\Uparrow}) \quad$ Figure 38

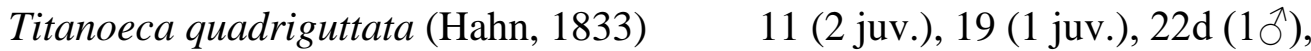

\section{Uloboridae}

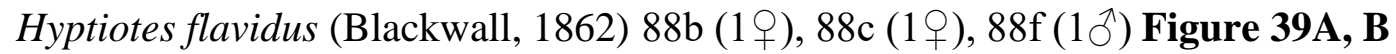

Uloborus plumipes Lucas, $1846 \quad 107$ a (2+)

Uloborus walckenaerius Latreille, $1806 \quad 22 \mathrm{c}(1+)$

\section{Zodariidae}

Zodarion frenatum Simon, $1884 \quad 58 \mathrm{~b}\left(1+2{ }^{\Uparrow}\right)$

Zodarion fuscum (Simon, 1870) $89(1 \overbrace{}^{\Uparrow}) \quad$ Figure 40A 
Zodarion italicum (Canestrini, 1868) 22a (1ठ)

Zodarion marginiceps Simon, 1914 110b (1우)

Zodarion morosum Denis, 1935

$61(1 \circ 1 \bigcirc)$

Zodarion rubidum Simon, 1914

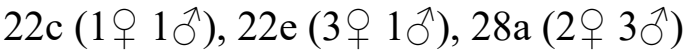

Zodarion rudyi Bosmans, 1994 $104(1 \bigcirc)$

Figure 40B

\section{Zoropsidae}

Zoropsis media Simon, 1878 47c (1), 47h (1ㅇ 1ठ)

Zoropsis spinimana (Dufour, 1820) 47a (1ㅇ)

\section{Conclusions}

The European material in the Duffey collection is interesting for a number of reasons. On the one hand, it illustrates the enormous progress made in the tools available for spider identification since the days his collection was assembled. While it previously would have been almost impossible to identify a good fraction of the specimens without recourse to reference material, the availability of the image collections of the Spiders of Europe keys and Pierre Oger's photographic documentation, as well as their tight integration with the World Spider Catalog for additional bibliographic references and immediate access to the entire relevant literature, mean that the identification work for South European spiders is now almost as easy as for material from Central and Northwestern Europe. On the other hand, the presence of a handful of unidentified specimens, possibly new species, 14 potentially new country records for (mainland) Spain, and numerous problematic cases for which only tentative identifications could be proposed, demonstrate the need for further faunistic exploration and taxonomic clarification. In several cases, only the availability of recent revisionary studies of difficult groups allowed the confident identification of Duffey's material. The importance of amateur collections in contributing to future advances in this direction cannot be overstated, and their deposition in museum collections for permanent safe keeping is strongly encouraged.

\section{Acknowledgements}

I am very grateful to the late Eric Duffey and his wife Rita for donating this valuable collection to the Manchester Museum, and to Dmitri Logunov (Manchester Museum) for entrusting the sorting and identification of the material to me, as well as providing the necessary facilities and advice throughout. I thank Barbara Thaler-Knoflach, Pierre Oger, Simeon Indzhov and participants of the spider forum of the Arachnologische Gesellschaft for helpful discussions of challenging identification problems. 
1500

1501

Bacelar A (1936) Notas aracnológicas. III. Aranhas ibéricas da familia Eresidae. Boletim da Sociedade Portuguesa de Ciencias Naturais 12:179-183.

Barrientos JA, Uribarri I \& García-Sarrión R (2015) Arañas (Arachnida, Araneae) del Turó de l'Home (Parc Natural del Montseny, Cataluña, España). Revista Ibérica de Aracnología 27: 61-74.

Benoit PLG (1974) Araignées intéressantes du Muséum d'Histoire Naturelle de Genève. Revue Suisse de Zoologie 81:993-998.

Bidegaray-Batista L, Sánchez-Gracia A, Santulli G, Maiorano L, Guisan A, Vogler AP \& Arnedo MA (2016) Imprints of multiple glacial refugia in the Pyrenees revealed by phylogeography and palaeodistribution modelling of an endemic spider. Molecular Ecology 25(9):2046-2064.

Binford GJ, Gillespie RG, Maddison WP (2016) Sexual dimorphism in venom chemistry in Tetragnatha spiders is not easily explained by adult niche differences. Toxicon 114:45-52. doi: 10.1016/j.toxicon.2016.02.015.

Bolzern A, Burckhardt D, Hänggi A (2013) Phylogeny and taxonomy of European funnelweb spiders of the Tegenaria-Malthonica complex (Araneae: Agelenidae) based upon morphological and molecular data. Zool J Linn Soc 168:723-848

Bosmans R, Kherbouche-Abrous O, Benhalima S \& Hervé C (2018) The genus Haplodrassus Chamberlin, 1922 in the Mediterranean and the Maghreb in particular (Araneae: Gnaphosidae). Zootaxa 4451(1):1-67. doi:10.11646/zootaxa.4451.1.1

Bosmans R, Hernández-Corral J \& Lissner J (2019) Description of a new Canariphantes species from the Balearic Islands (Araneae: Linyphiidae). Arachnology 18(2): 97-100. doi:10.13156/arac.2018.18.2.97

Branco V V (2019) An update to the Iberian spider checklist (Araneae). Zootaxa 4614:201254. doi: 10.11646/zootaxa.4614.2.1

Brignoli P M (1978) Quelques notes sur les Agelenidae, Hahniidae, Oxyopidae et Pisauridae de France et d'Espagne (Araneae). Revue suisse Zool 85:265-294

Crespo LC, Domènech M, Enguídanos A, Malumbres-Olarte J, Cardoso P, Moya-Laraño J, Frías-López C, Macías-Hernández N, De Mas E, Mazzuca P, Mora E, Opatova V, Planas E, Ribera C, Roca-Cusachs M, Ruiz D, Sousa P, Tonzo V, Arnedo MA (2018) A DNA barcodeassisted annotated checklist of the spider (Arachnida, Araneae) communities associated to white oak woodlands in Spanish National Parks. Biodiversity Data Journal 6(e29443):1-459

Dalmas R de (1920) Liste d'araignées de Boudron en Asie Mineure suive d'une étude des espèces méditerranéennes du genre Habrocestum. Annali del Museo Civico di Storia Naturale di Genova 50:57-69. 
Deltshev CD (1992) A critical review of family Theridiidae (Araneae) in Bulgaria. Acta Zoologica Bulgarica 43: 13-21.

Denis J (1934) Sur quelques araignées des Pyrenees-Orientales. Bulletin de la Société Entomologique de France 39: 72-77.

Denis J (1945) Sur quelques Theridion appartenant à la faune de France. Bulletin de la Société Entomologique de France 49(1944): 111-117.

Denis J (1965) Notes sur les érigonides. XXVIII Le genre Trichoncus (Araneae). Annales de la Société Entomologique de France (N.S.) 1: 425-477.

Duffey E \& Brignoli PM (1981) Two rare spiders from the Spanish Pyrenees (prov. Huesca). Bulletin of the British Arachnological Society 5:155-158.

Foord SH (2008) Cladistic analysis of the Afrotropical Hersiliidae (Arachnida, Araneae) with the first records of Murricia and the description of a new genus from Madagascar. Journal of Afrotropical Zoology 4:111-142.

Foord SH \& Dippenaar-Schoeman AS (2005) A revision of the Afrotropical species of Hersiliola Thorell and Tama Simon with the description of a new genus Tyrotama (Araneae: Hersiliidae). African Entomology 13:255-279.

Grimm U (1985) Die Gnaphosidae Mitteleuropas (Arachnida, Araneae). Abhandlungen des Naturwissenschaftlichen Vereins in Hamburg 26: 1-318.

Hansen H (1995) Über die Arachniden-Fauna von urbanen Lebensräumen in Venedig - III. Die epigäischen Spinnen eines Stadtparkes (Arachnida: Araneae). Bollettino del Museo Civico di Storia Naturale di Venezia 44(for 1993): 7-36.

Isaia M, Paschetta M, Chiarle A (2015) Annotated checklist of the spiders (Arachnida, Araneae) of the Site of Community Importance and Special Area of Conservation "Alpi Marittime” (NW Italy), IN: Daugeron, C., Deharveng, L., Isaia, M., Villemant, C. \& Judson, M. (eds), Mercantour/Alpi Marittime All Taxa Biodiversity Inventory. Zoosystema, 37(1):57-114. https://doi.org/10.5252/z2015n1a4

Jocqué R (1993) A new species of Coriarachne from Spain (Araneae, Thomisidae). Bulletin de l'Institut Royal des Sciences Naturelles de Belgique (Ent.) 63:119-122.

Kiany N, Sadeghi S, Kiany M, Zamani A \& Ostovani S (2017) Additions to the crab spider fauna of Iran (Araneae: Thomisidae). Arachnologische Mitteilungen 53:1-8. doi:10.5431/aramit5301

Koch L (1877) Verzeichniss der bei Nürnberg bis jetzt beobachteten Arachniden (mit Ausschluss der Ixodiden und Acariden) und Beschreibungen von neuen, hier vorkommenden Arten. Abhandlungen der Naturhistorischen Gesellschaft zu Nürnberg 6: 113-198. 
Kulczyński V (1911) Fragmenta Arachnologica, IX. Bulletin International de l'Académie des Sciences de Cracovie 1911:12-75.

Lecigne S (2012) Inventaire aranéologique (Arachnida, Araneae) dans la ville d'Estepona (Malaga, Espagne). Revista Ibérica de Aracnología 21:161-167.

Lecigne S (2016) Contribution à la connaissance de l'aranéofaune (Araneae) de Crète (Grèce) et description de la femelle inconnue de Neaetha absheronica Logunov \& Guseinov, 2002 (Salticidae). Nieuwsbrief van de Belgische Arachnologische Vereniging 30(3):95-118.

Lecigne S, Cornic J-F, Oger P \& Van Keer J (2019) Celerrimus n. gen. (Araneae, Philodromidae) et description de Celerrimus duffeyi n. sp., une espèce très singulière d'Europe occidentale. Revue Arachnologique (2) 6: 32-51.

Lemke M, E Merches \& A Hänggi (2014) Neue Arbeitsgruppe der Arachnologischen Gesellschaft (AraGes) mit Erweiterung der Webpräsenz: Arachniden-Wiki und -Forum. Arachnologische Mitteilungen 48:8-9.

Le Peru B (2011) The spiders of Europe, a synthesis of data: Volume 1 Atypidae to Theridiidae. Mémoires de la Société Linnéenne de Lyon 2:1-522.

Levy G (1976) The spider genus Xysticus (Araneae: Thomisidae) in Israel. Israel Journal of Zoology 25:1-37.

Levy G (2003) Spiders of the families Anyphaenidae, Hahniidae, Ctenidae, Zoridae, and Hersiliidae (Araneae) from Israel. Israel Journal of Zoology 49:1-31.

Machado A de B (1941) Araignées nouvelles pour la faune portugaise (II). Memorias e Estudos do Museu Zoologico da Universidade de Coimbra 117:i-xvi, 1-60.

Marusik YM \& Logunov DV (1995) The crab spiders of Middle Asia (Aranei, Thomisidae), 2. Beiträge zur Araneologie 4:133-175.

Marusik YM \& Fet V (2009) A survey of east Palearctic Hersiliola Thorell, 1870 (Araneae, Hersiliidae), with a description of three new genera. ZooKeys 16:75-114. doi:10.3897/zookeys.16.229

Muff P, Schmidt MH, Frick H, Nentwig W \& Kropf C (2007) Spider (Arachnida: Araneae) distribution across the timberline in the Swiss Central Alps (Alp Flix, Grisons) and three morphologically variable species. Arachnologische Mitteilungen 34: 16-24.

Paschetta M, La Morgia V, Masante D, Negro M, Rolando A, \& Isaia M (2013) Grazing history influences biodiversity: a case study on ground-dwelling arachnids (Arachnida: Araneae, Opiliones) in the Natural Park of Alpi Marittime (NW Italy). Journal of Insect Conservation 17(2): 339-356.

Prószyński J (1987). Atlas rysunków diagnostycznych mniej znanych Salticidae 2. Zeszyty Naukowe Wyższej Szkoly Rolniczo-Pedagogicznej, Siedlce, 172 pp. 
Řezáč M, Pekár S \& Johannesen J (2008) Taxonomic review and phylogenetic analysis of central European Eresus species (Araneae: Eresidae). Zoologica Scripta 37(3):263-287. doi:10.1111/j.1463-6409.2008.00328.x

Ribera C, Ferrández MÁ \& Pérez JA (1988) Los hersílidos (Araneae, Hersiliidae) de la fauna Ibérica. Miscellanea Zoologica 10:97-103.

Schenkel E (1938) Spinnentiere von der Iberischen Halbinsel, gesammelt von Prof. Dr O. Lundblad, 1935. Arkiv för Zoologi 30(A24): 1-29.

Thaler K (1981) Bemerkenswerte Spinnenfunde in Nordtirol (Österreich). Veröffentlichungen des Museum Ferdinandeum in Innsbruck 61: 105-150.

Tuneva TK \& Esyunin SL (2003) A review of the family Gnaphosidae in the fauna of the Urals (Aranei), 4. The first record of Gnaphosa tigrina Simon, 1878 and remarks on two species from the rufula group. Arthropoda Selecta 11: 277-281.

Tyschchenko VP (1971) Opredelitel' paukov evropejskoj chasti SSSR. Leningrad, pp. 1-281.

Vanuytven H, Van Keer J \& Poot P (1994) Kogelspinnen verzameld in Zuid-Europa door P. Poot (Araneae, Theridiidae). Nieuwsbrief van de Belgische Arachnologische Vereniging 9: 119.

Wiehle H (1933) Holocnemus hispanicus sp. n. und die Gattungen Holocnemus Simon und Crossoprisa Simon. Zoologischer Anzeiger 104: 241-252.

Wiehle H (1937) Spinnentiere oder Arachnoidea. 26. Familie. Theridiidae oder Haubennetzspinnen (Kugelspinnen). Die Tierwelt Deutschlands 33: 119-222.

Wiehle H (1960a) Der Embolus des männlichen Spinnentasters. Verhandlungen der Deutschen Zoologischen Gesellschaft 1960:457-480.

Wiehle H (1960b) Beiträge zur Kenntnis der deutschen Spinnenfauna. Zoologische Jahrbücher, Abteilung für Systematik, Geographie und Biologie der Tiere 88: 195-254.

World Spider Catalog (2019) World Spider Catalog. Version 19.5. Natural History Museum Bern, online at http://wsc.nmbe.ch, accessed on 01 April 2019. doi: 10.24436/2

Wunderlich J (2011) Extant and fossil spiders (Araneae). Beiträge zur Araneologie 6: 1-640.

Zobel-Thropp PA, Bulger EA, Cordes MHJ, Binford GJ, Gillespie RG, Brewer MS (2018) Sexually dimorphic venom proteins in long-jawed orb-weaving spiders (Tetragnatha) comprise novel gene families. PeerJ. 6:e4691. doi: 10.7717/peerj.4691 


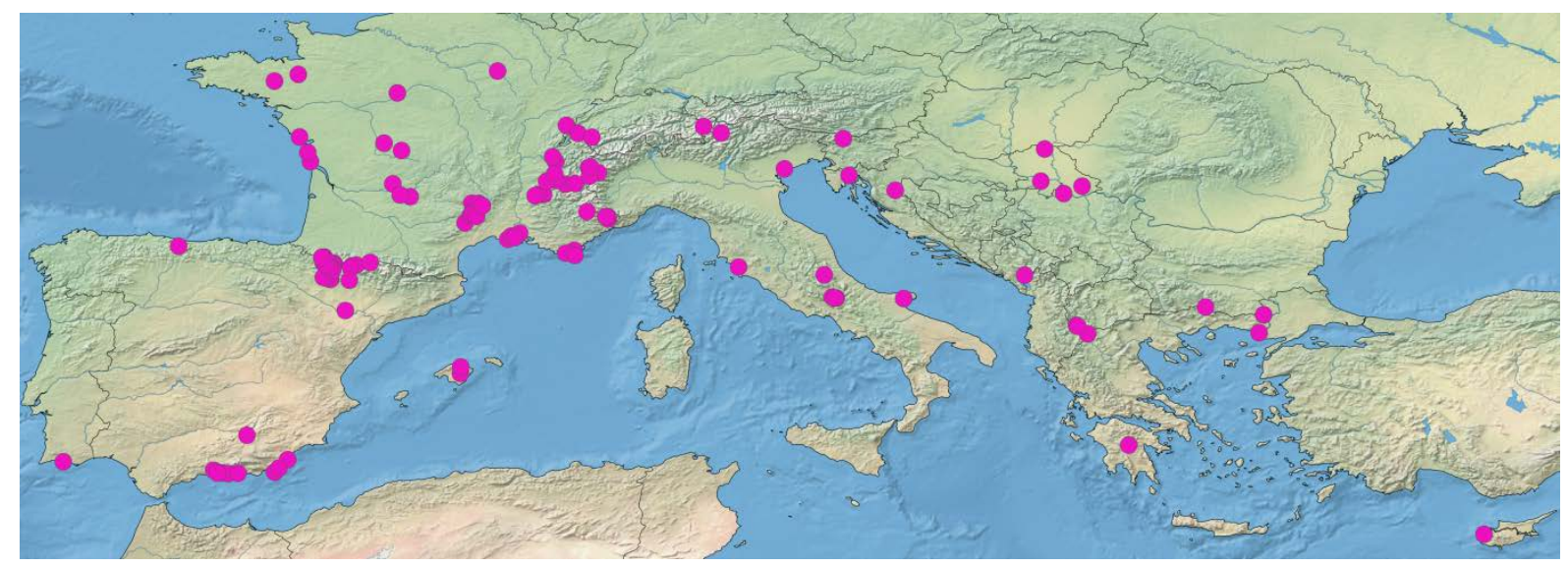

Figure 1. Overview of the South European sampling locations represented in the previously unidentified material of the Duffey collection in the Manchester Museum. 

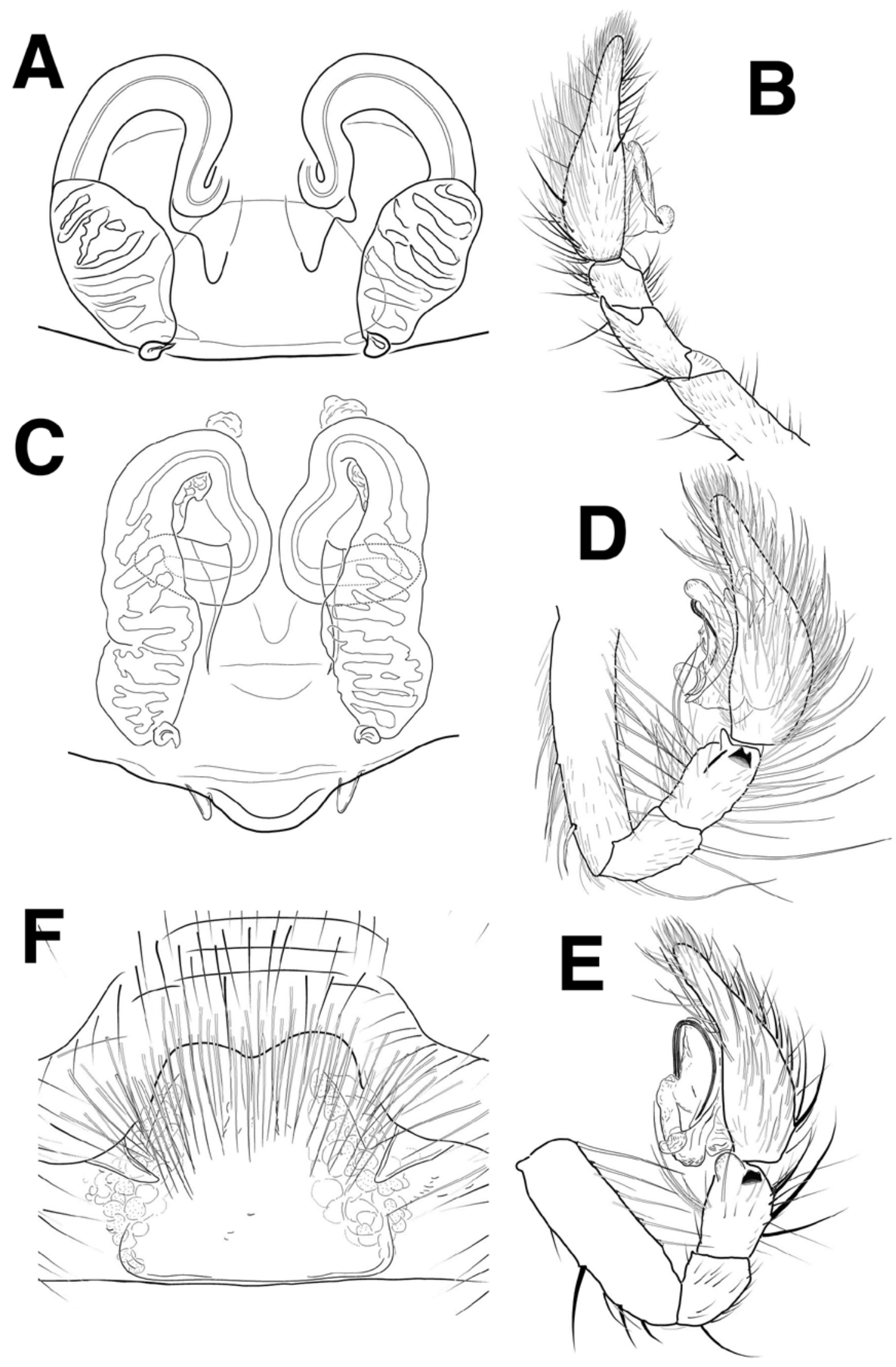

Figure 2. Agelenidae. A Eratigena bucculenta, vulva. B Eratigena feminea pedipalp, retrolateral. C, D Eratigena fuesslini vulva (C) and pedipalp, retrolateral (D). E Malthonica lusitanica pedipalp, retrolateral. F Pireneitega segestriformis epigynum. 


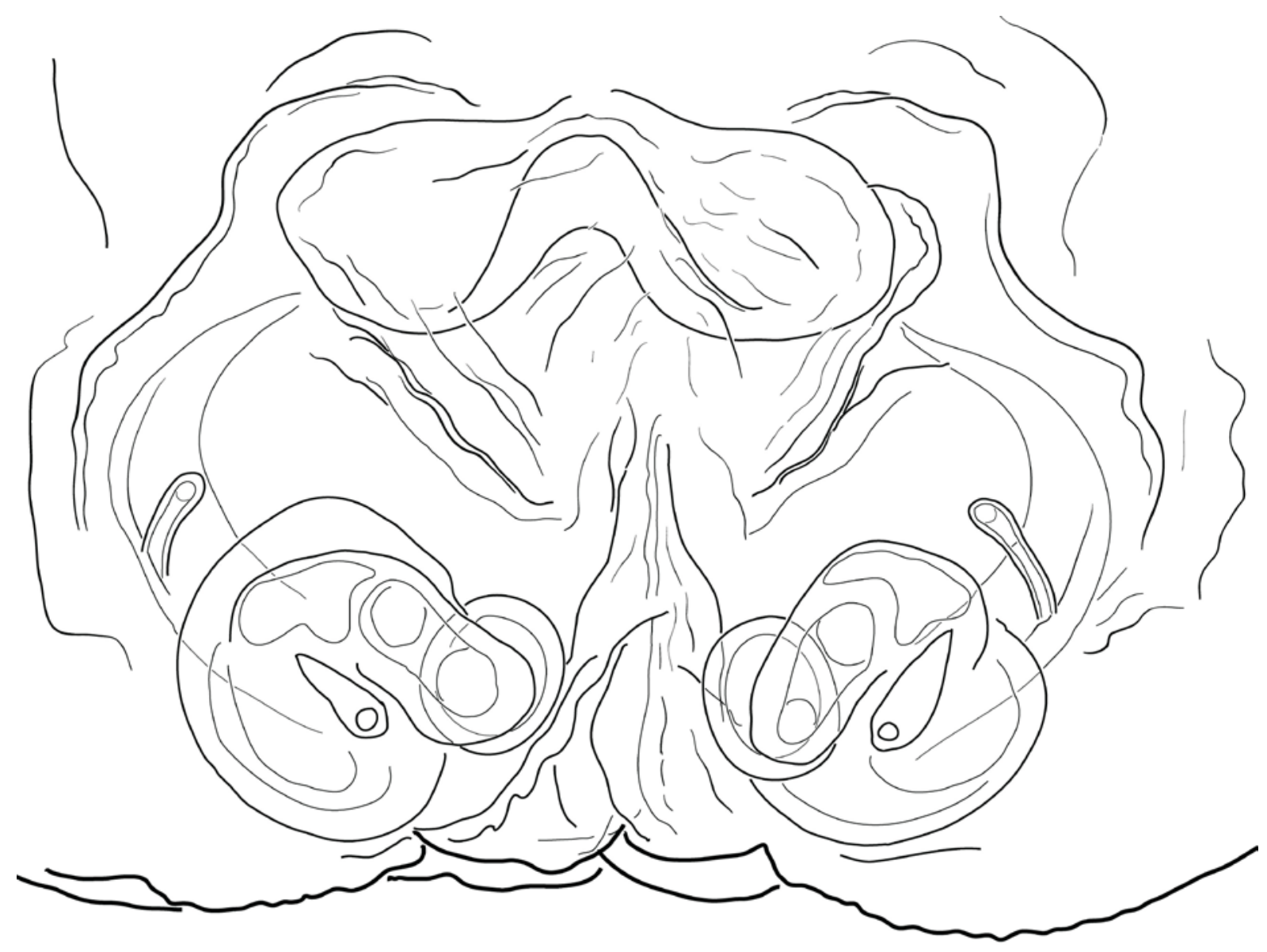

Figure 3. Anyphaenidae. Anyphaena sabina vulva. 


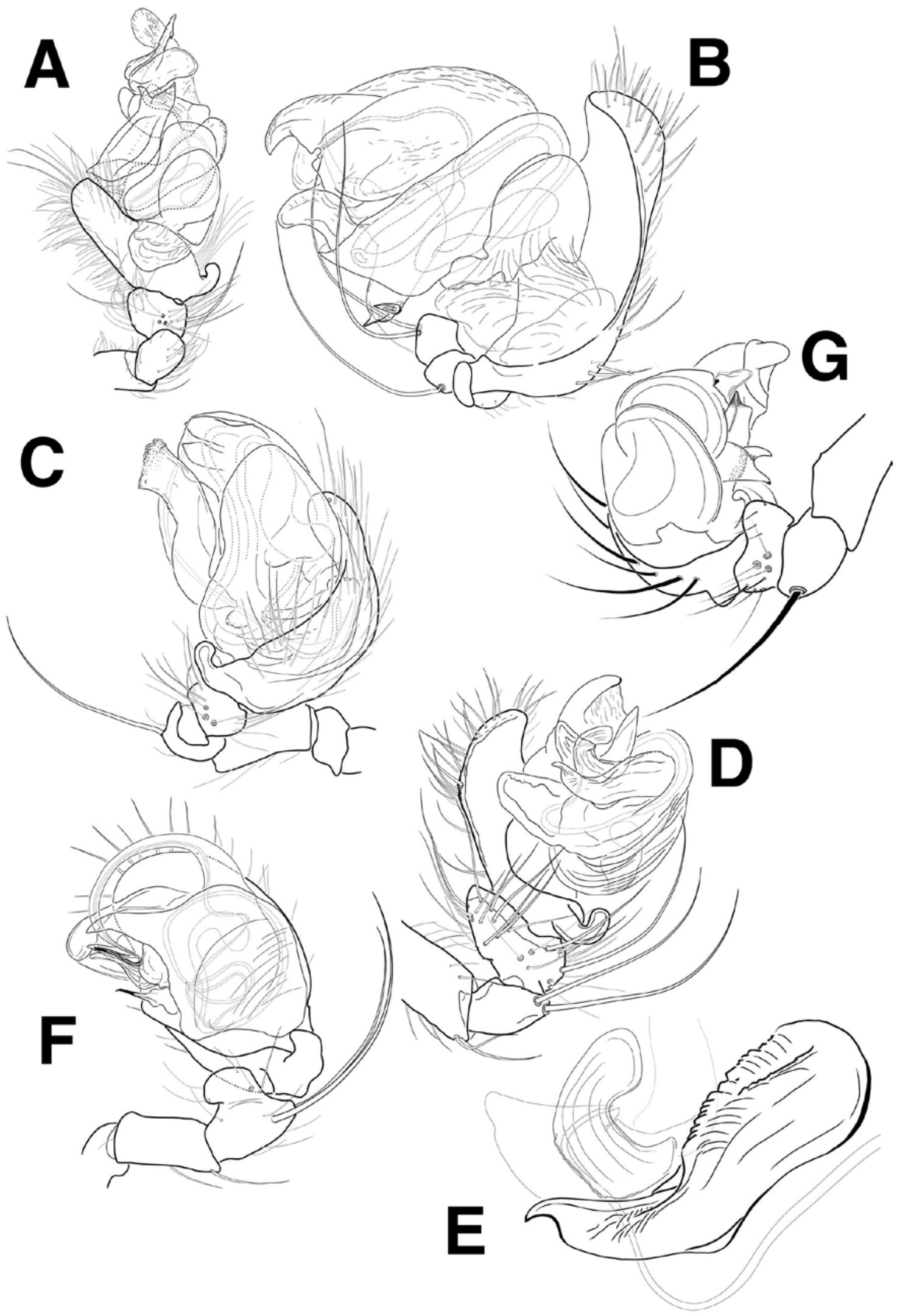

Figure 4. Araneidae males. A Araneus quadratus pedipalp, expanded. B Araneus sturmi pedipalp, slightly expanded. C Cyclosa conica pedipalp. D, E Gibbaranea gibbosa pedipalp, slightly expanded (D) and median apophysis (E). F Hypsosinga sanguinea pedipalp. G Mangora acalypha pedipalp. 


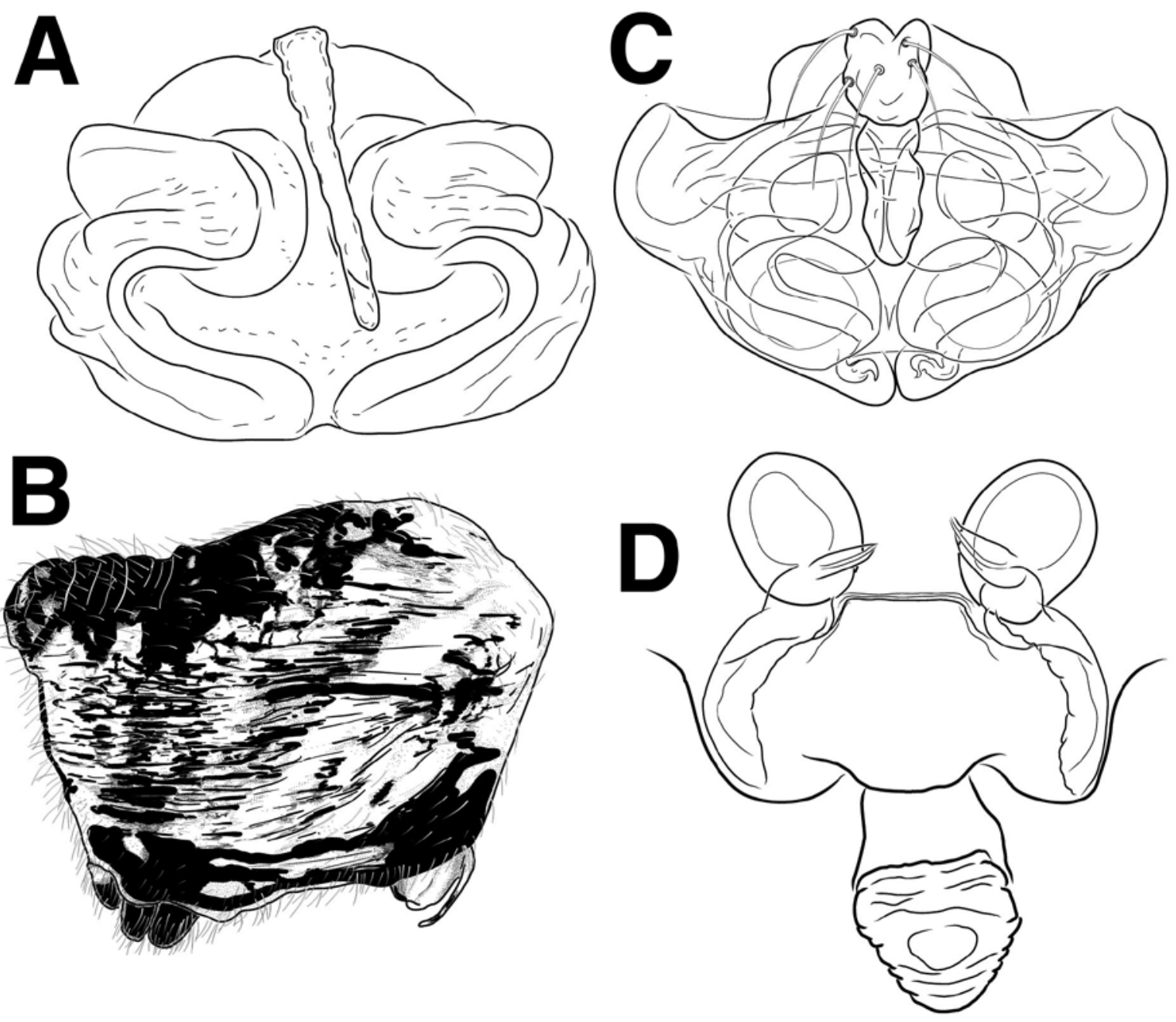

Figure 5. Araneidae females. A, B Cyclosa algerica epigynum (A) and habitus (B). C Cyclosa conica epigynum. D Mangora acalypha epigynum. 


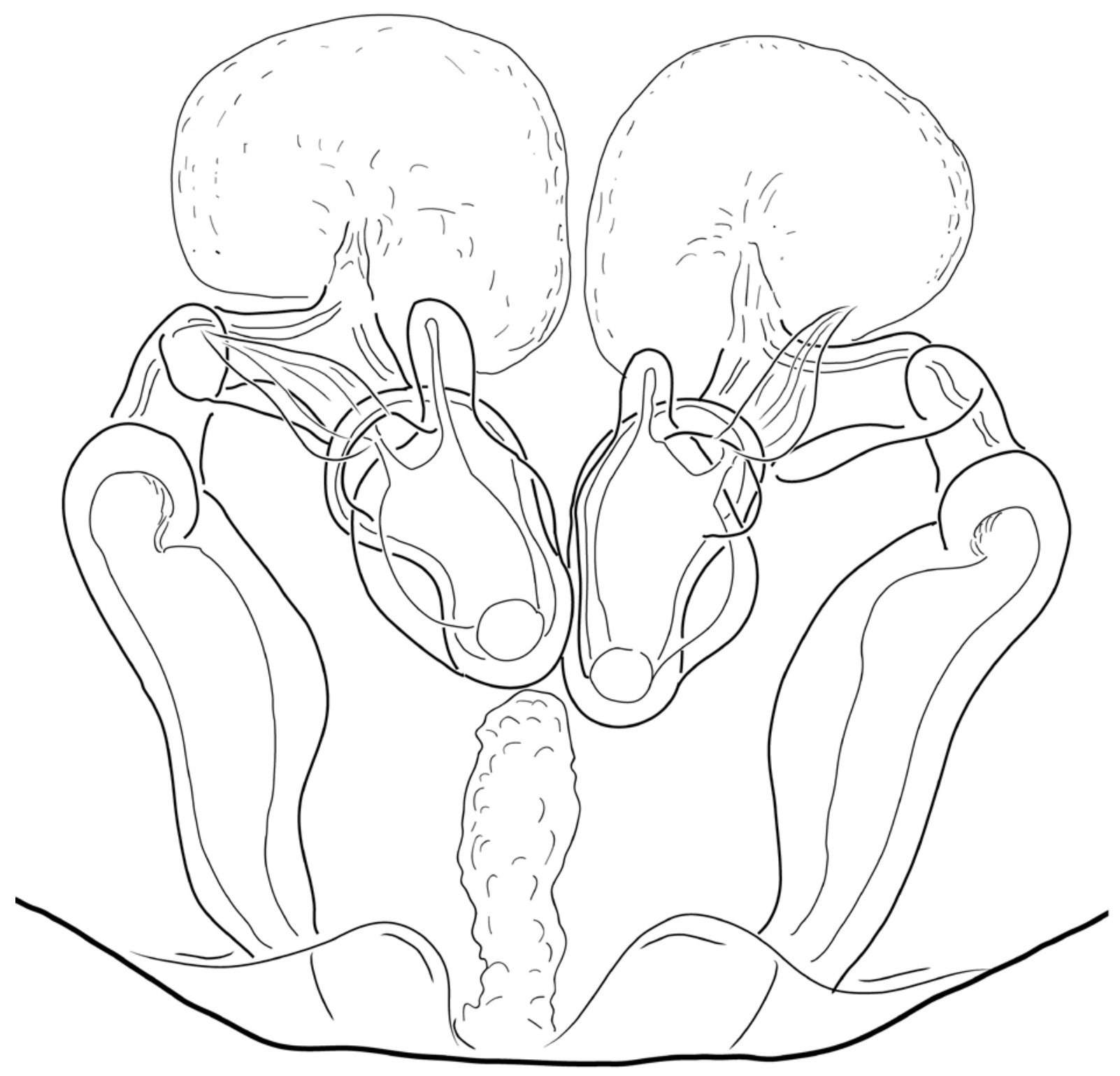

Figure 6. Clubionidae. Clubiona terrestris vulva. 

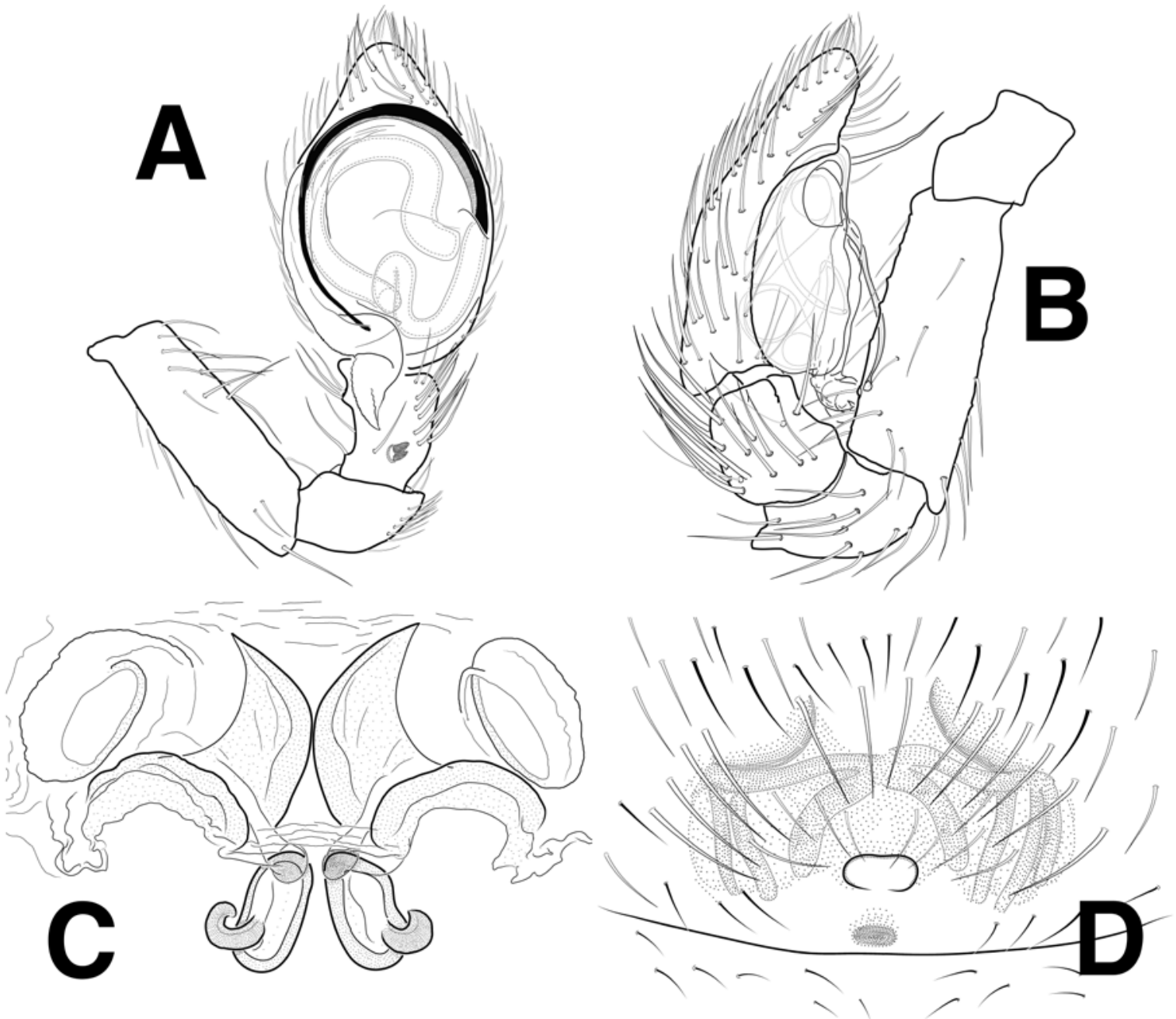

Figure 7. Dictynidae. A Brigittea latens pedipalp, ventral. B Nigma gratiosa pedipalp, retrolateral. C Nigma puella vulva. D (Scoto)Lathys sp. epigynum. 

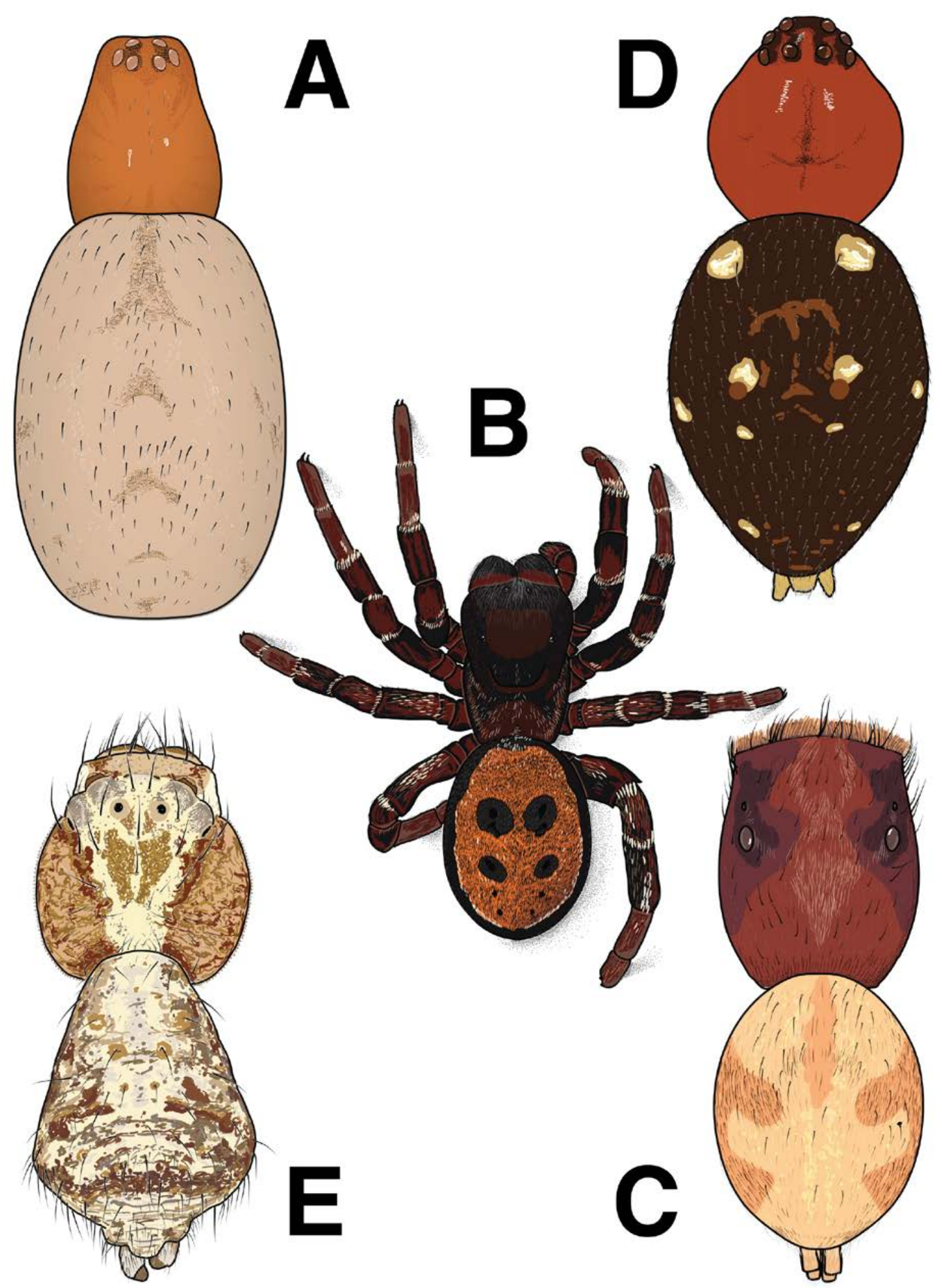

Figure 8. Habitus drawings. A (Scoto)Lathys sp. female. B Eresus cf. solitarius male. C Habrocestum cf. ibericum male. D Euryopis margaritata male. E Tmarus stellio female. 

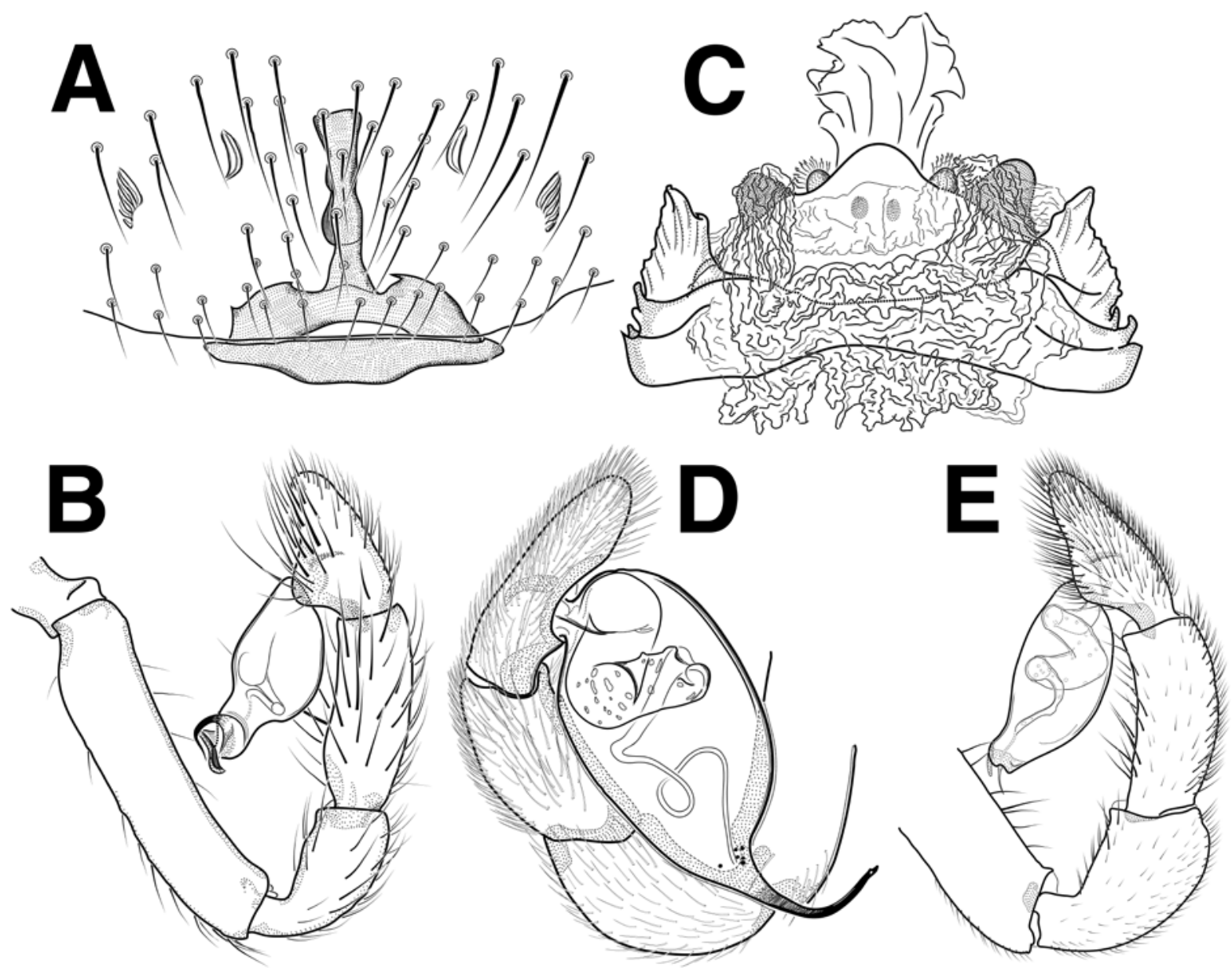

Figure 9. Dysderidae. A, B Harpactea hombergi endogynum, ventral (A) and pedipalp (B). C, D Harpactocrates ravastellus endogynum, dorsal (C) and pedipalp (D). E Parachtes teruelis pedipalp. 


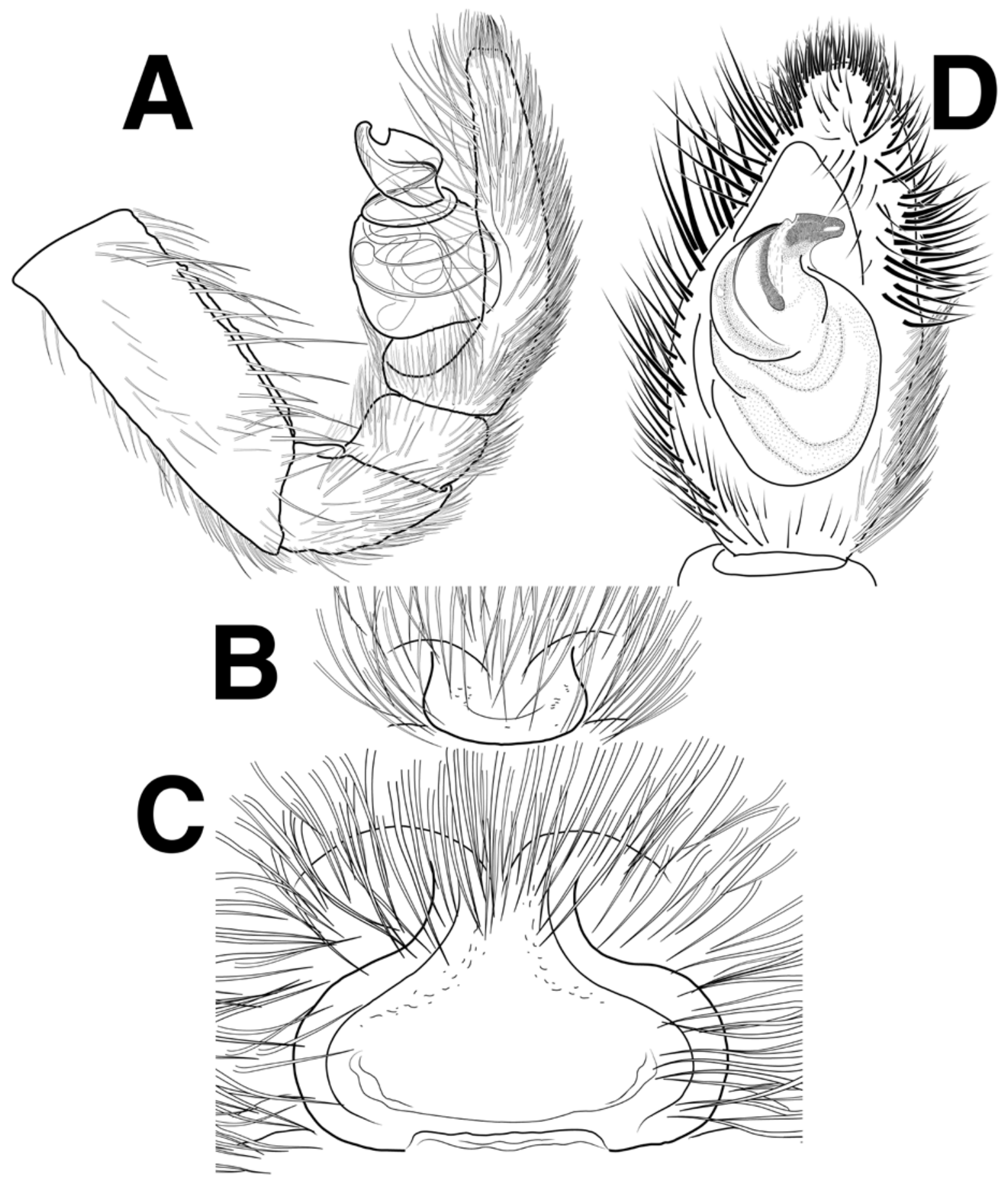

Figure 10. Eresidae. A Eresus cf. solitarius pedipalp. B-D Stegodyphus lineatus preepigynum (B), epigynum (C) and pedipalp (D). 


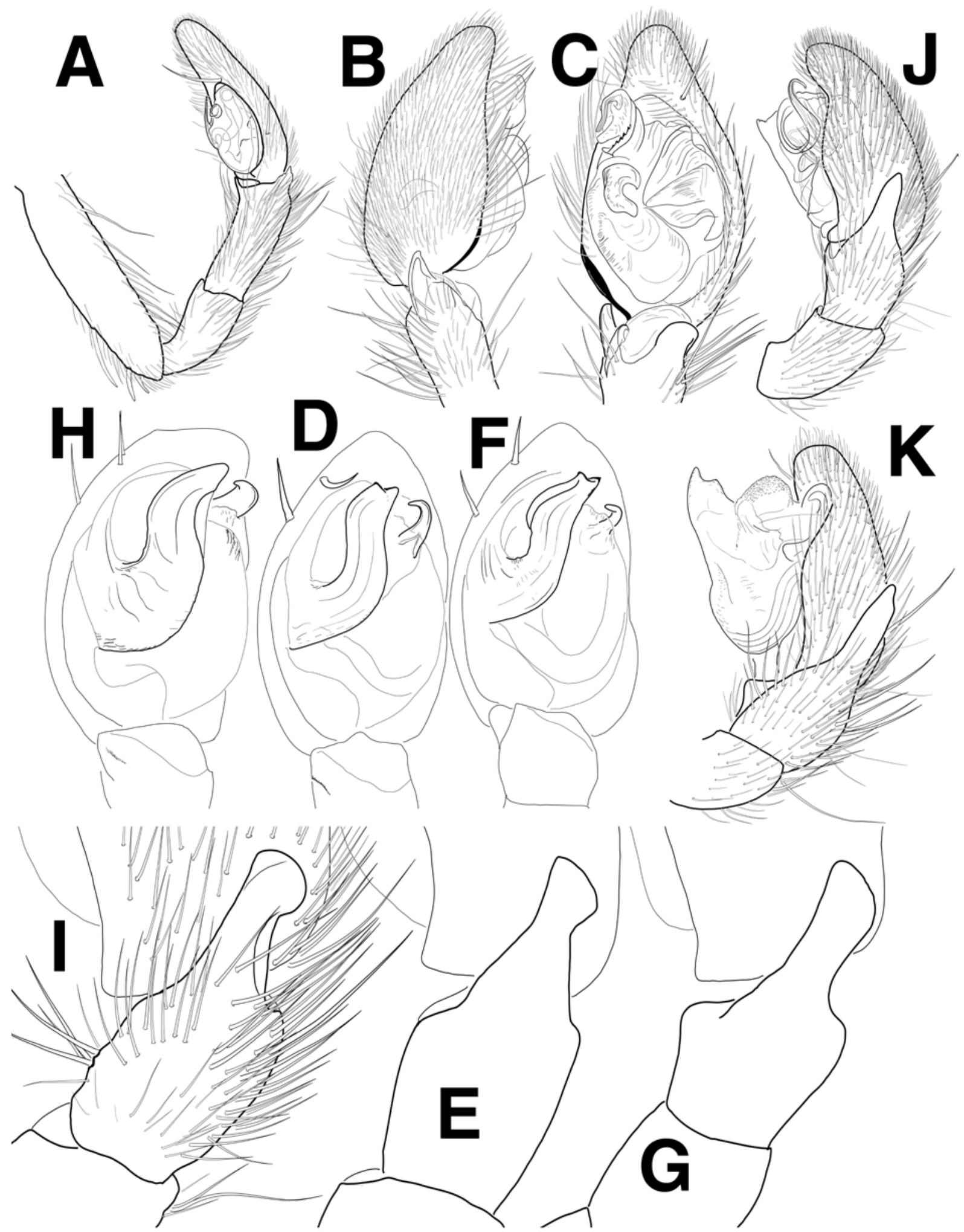

Figure 11. Gnaphosidae males. A Drassodes lapidosus pedipalp, retrolateral. B, C Drassodex fritillifer pedipalp retrolateral (B) and ventral (C). D, E Haplodrassus lyndae pedipalp ventral (D) and retrolateral tibial apophysis (RTA, E). F, G Haplodrassus rufipes pedipalp ventral (F) and RTA (G). H, I Haplodrassus signifer pedipalp ventral (H) and RTA (I). J Trachyzelotes mutabilis pedipalp, retrolateral. $\mathbf{K}$ Zelotes manius pedipalp, retrolateral. 

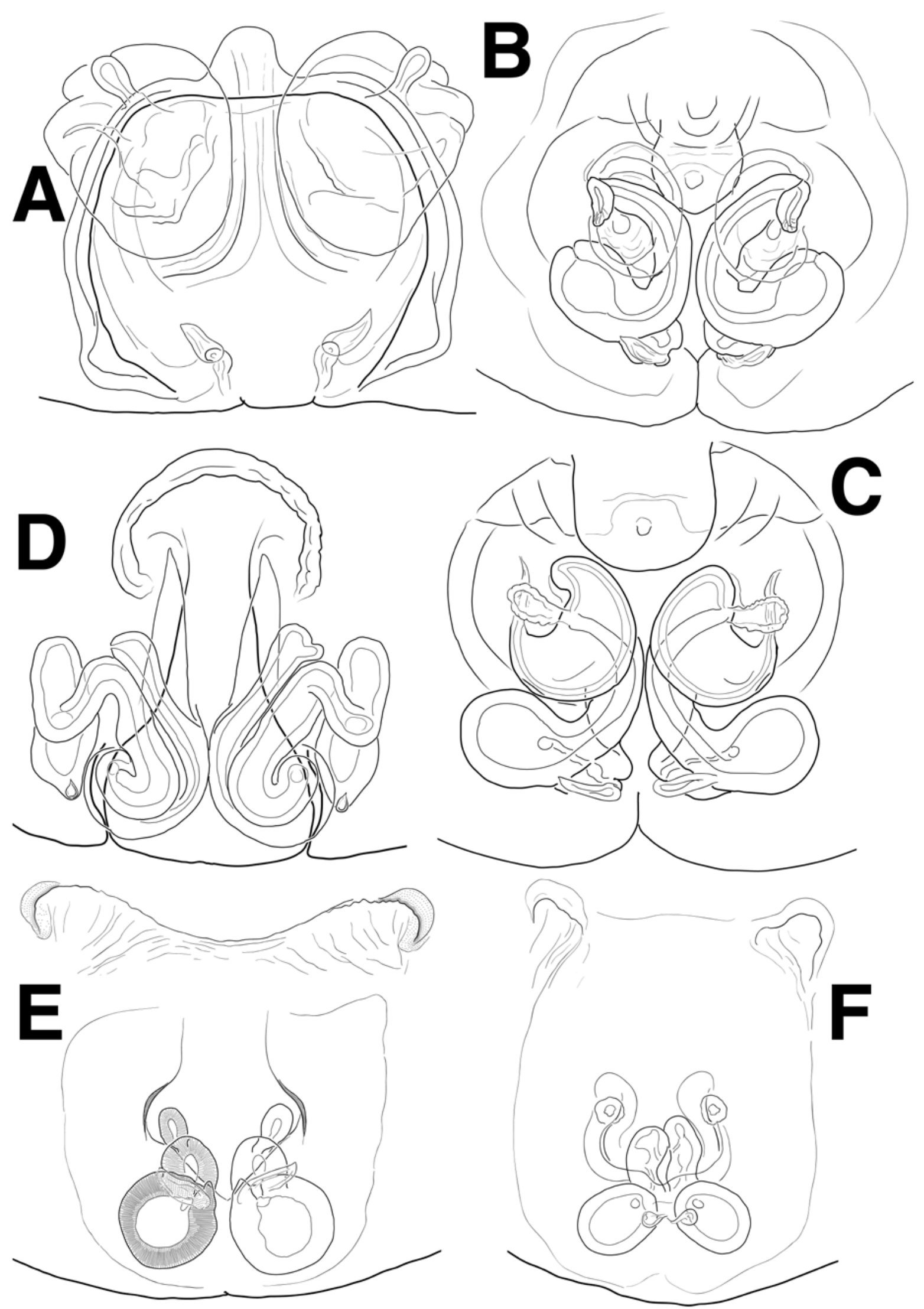

Figure 12. Gnaphosidae female vulvae A Drassodex hispanus. B Gnaphosa occidentalis. C Gnaphosa tigrina. D Pterotricha simoni. E Zelotes latreillei. F Zelotes manius. 

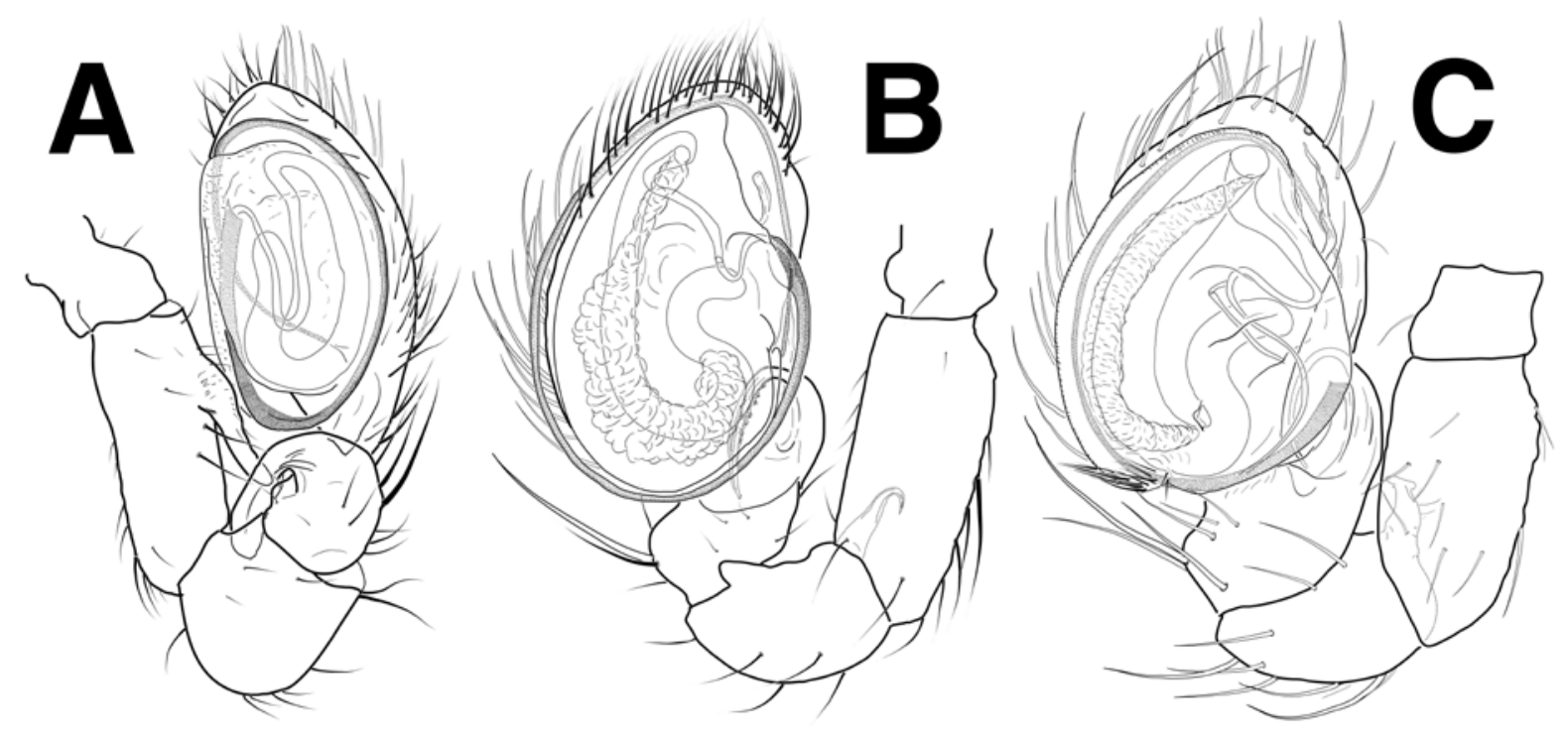

Figure 13. Hahniidae male pedipalps. A Antistea elegans. B Hahnia nava. C Hahnia ononidum.

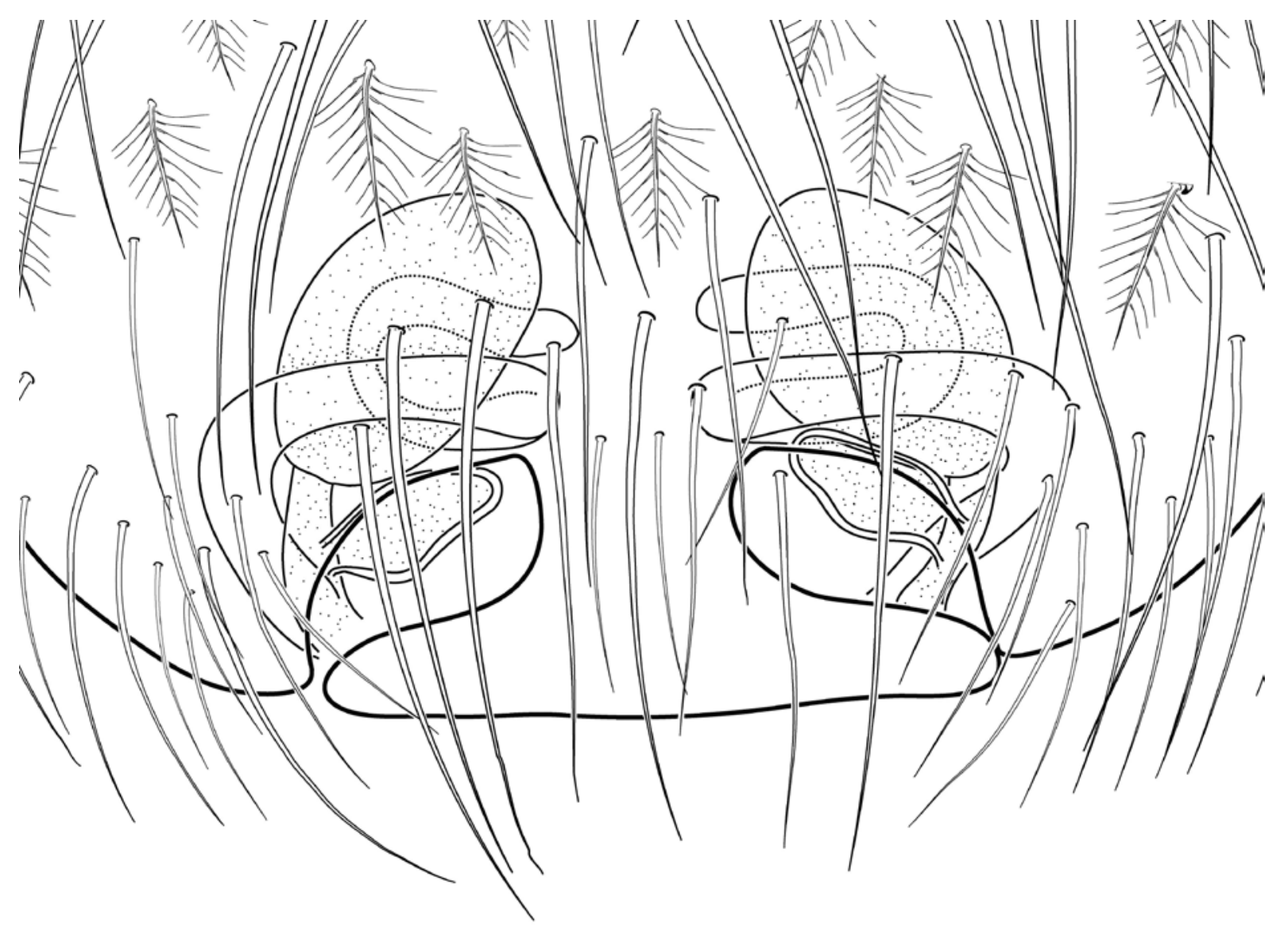

Figure 14. Hersiliidae. Hersiliola cf. simoni epigynum. 


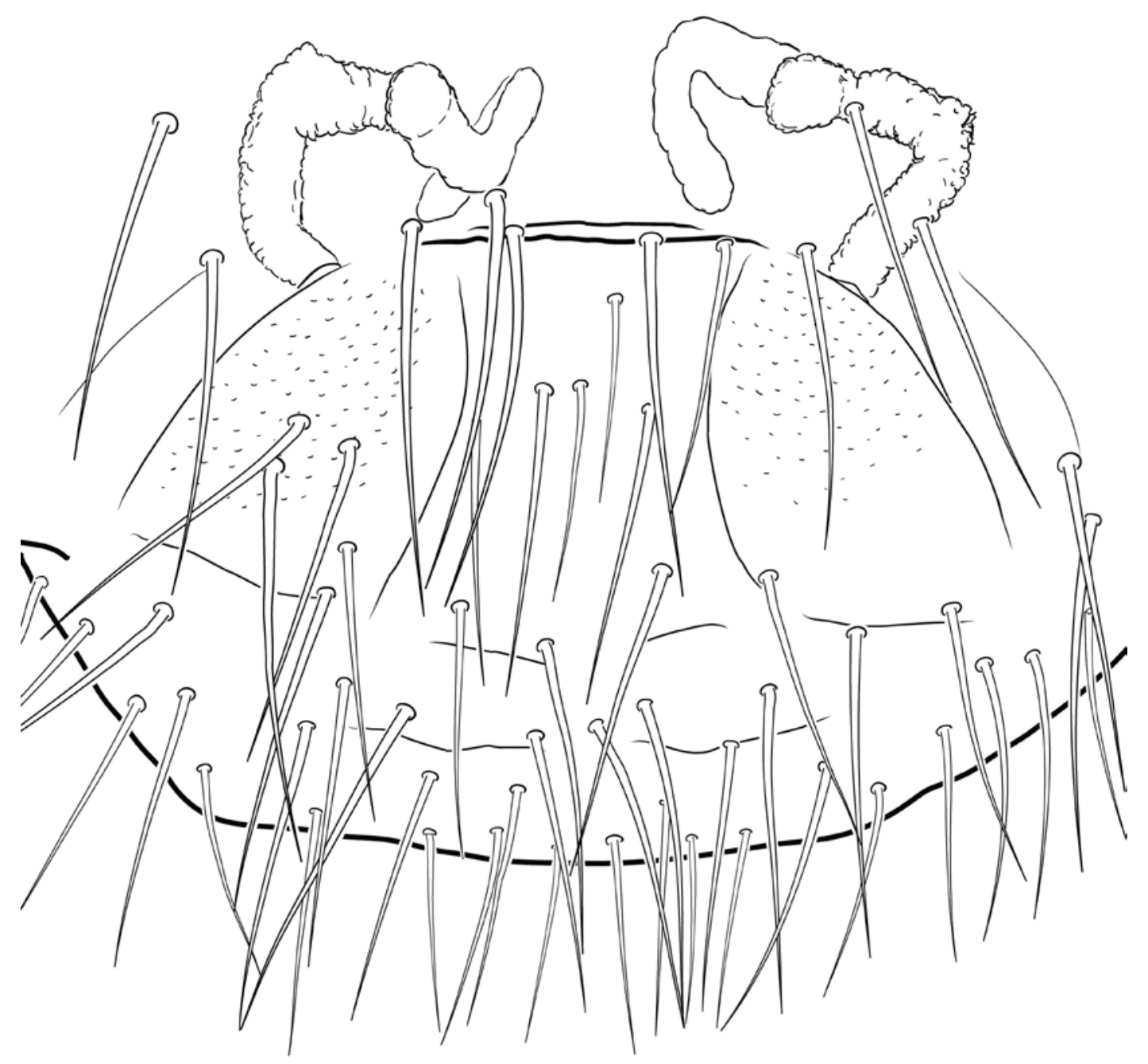

Figure 15. Leptonetidae. Leptoneta paroculus endogynum, ventral. 

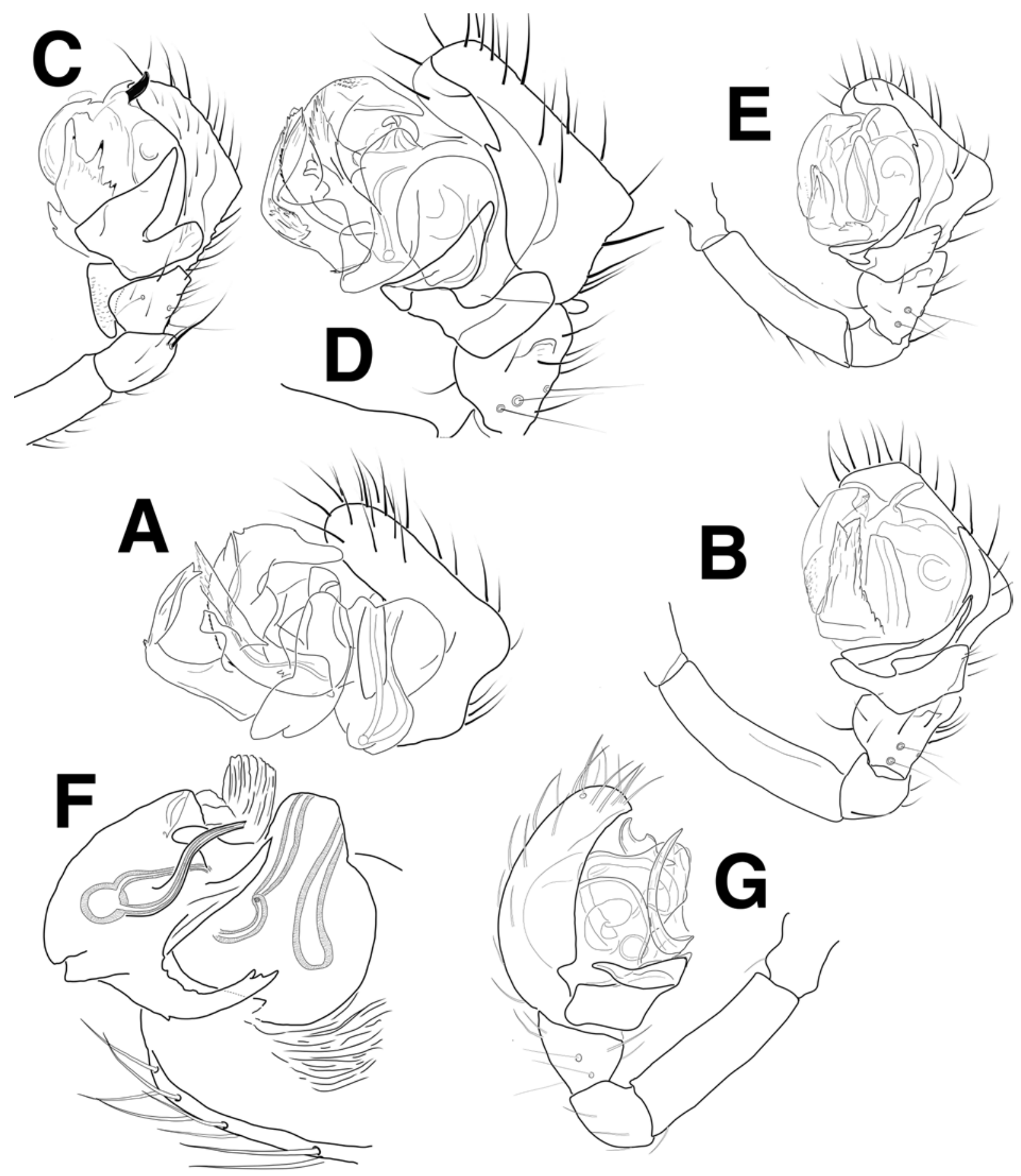

Figure 16. Linyphiidae males. A+B Agyneta cf. alpica expanded bulbus (A) and pedipalp retrolateral (B). C Agyneta equestris pedipalp retrolateral. D+E Agyneta rurestris expanded bulbus (D) and pedipalp retrolateral (E). F Canariphantes tenerrimus embolic division expanded. G Canariphantes zonatus pedipalp retrolateral. 


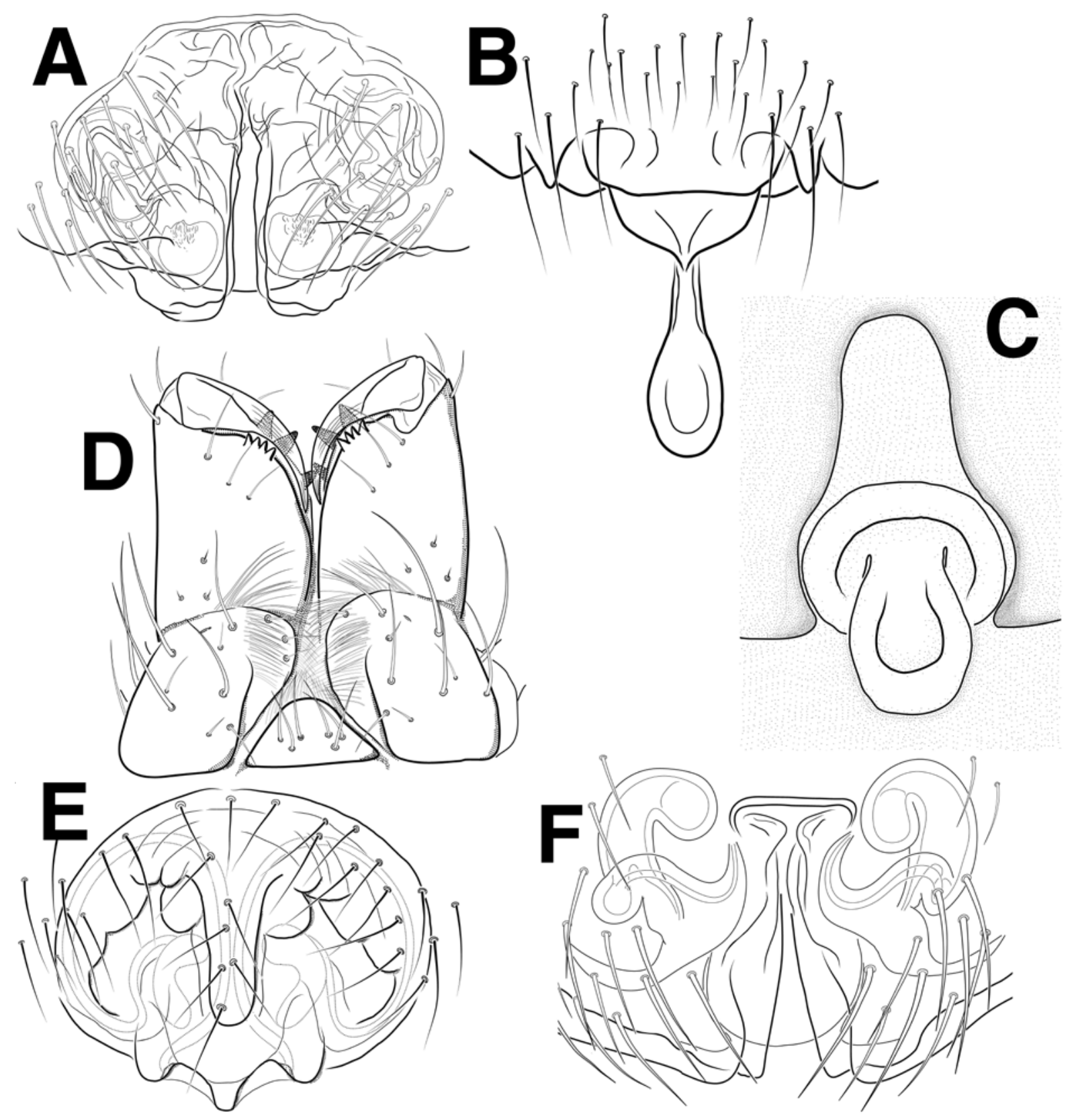

Figure 17. Linyphiidae females. A Areoncus anguineus epigynum. B Canariphantes ritae epigynum. C Canariphantes tenerrimus epigynum. D+E Centromerus albidus chelicerae (D) and epigynum (E). F Diplocephalus protuberans epigynum. 

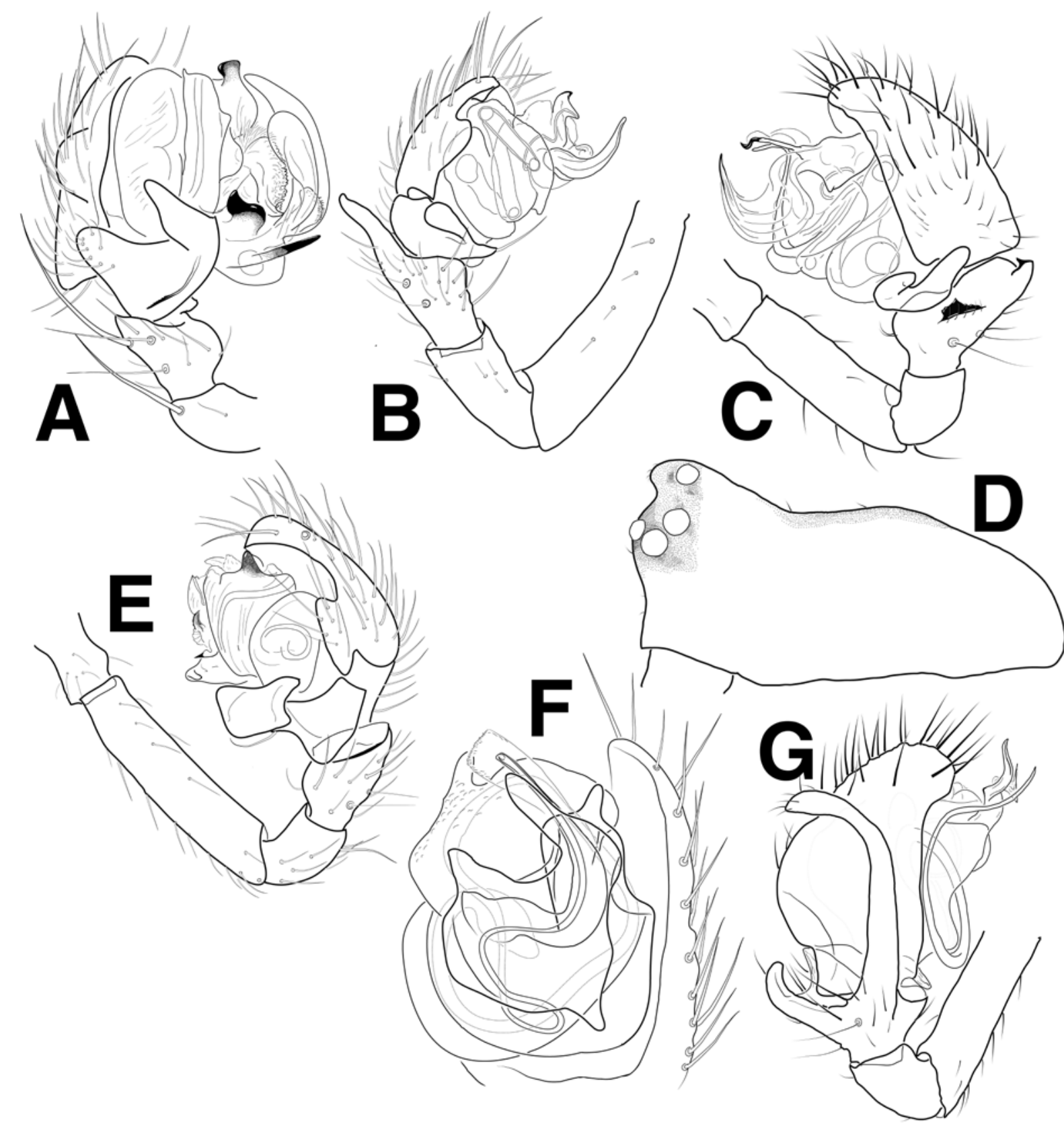

Figure 18. Linyphiidae males. A Centromerus pabulator pedipalp retrolateral, slightly expanded. B Hilaira excisa pedipalp retrolateral. C+D Metopobactrus prominulus $\mathrm{f}$. schenkeli pedipalp (C) and prosoma (D). E Oreonetides glacialis pedipalp retrolateral. F Porrhomma convexum pedipalp expanded. G Trichoncus saxicola pedipalp dorsal. 

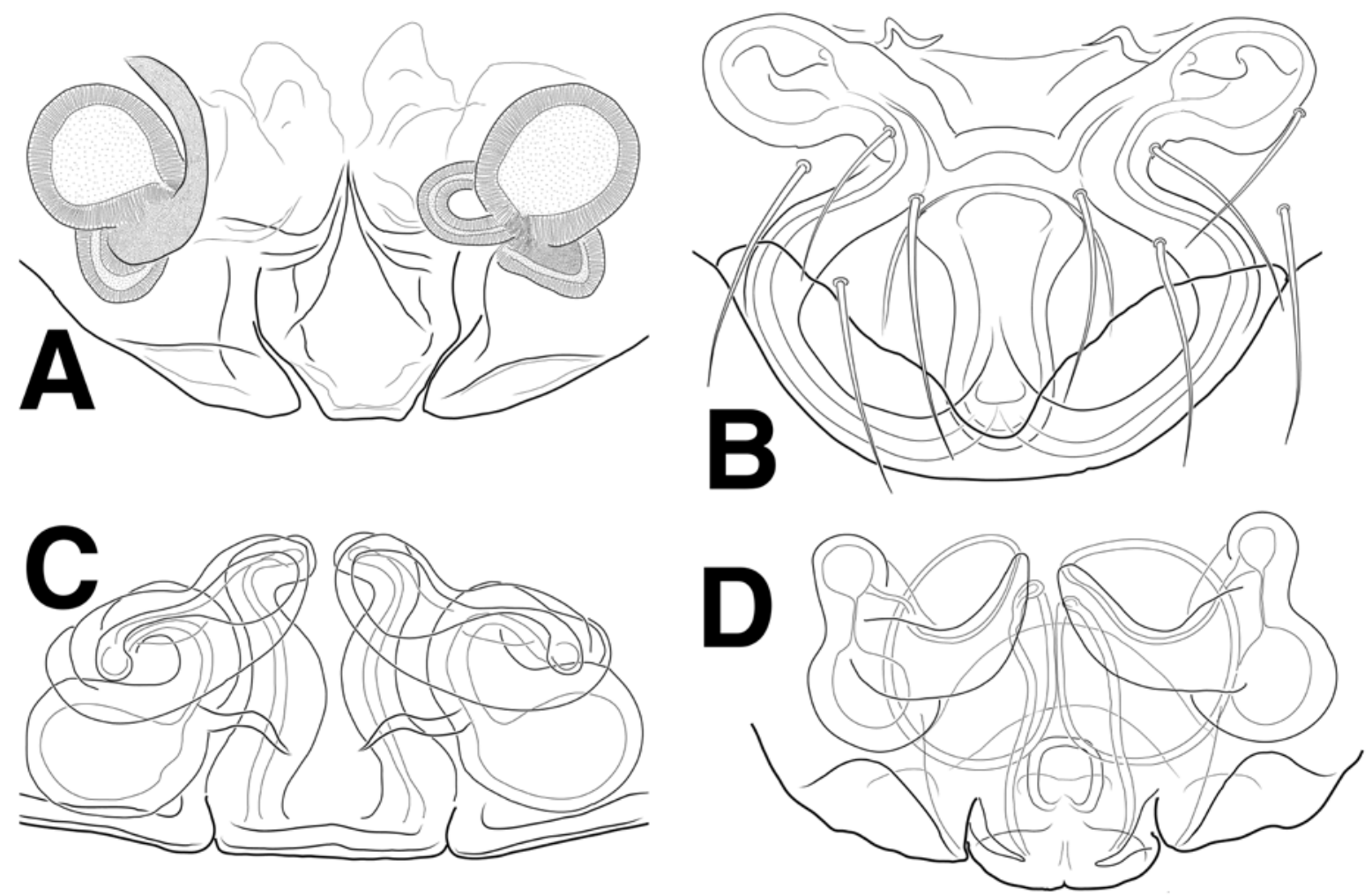

Figure 19. Linyphiidae females. A Mecopisthes nicaeensis vulva. B Oreonetides glacialis epigynum. C Pelecopsis bucephala vulva. D Trichoncus cf. auritus vulva. 


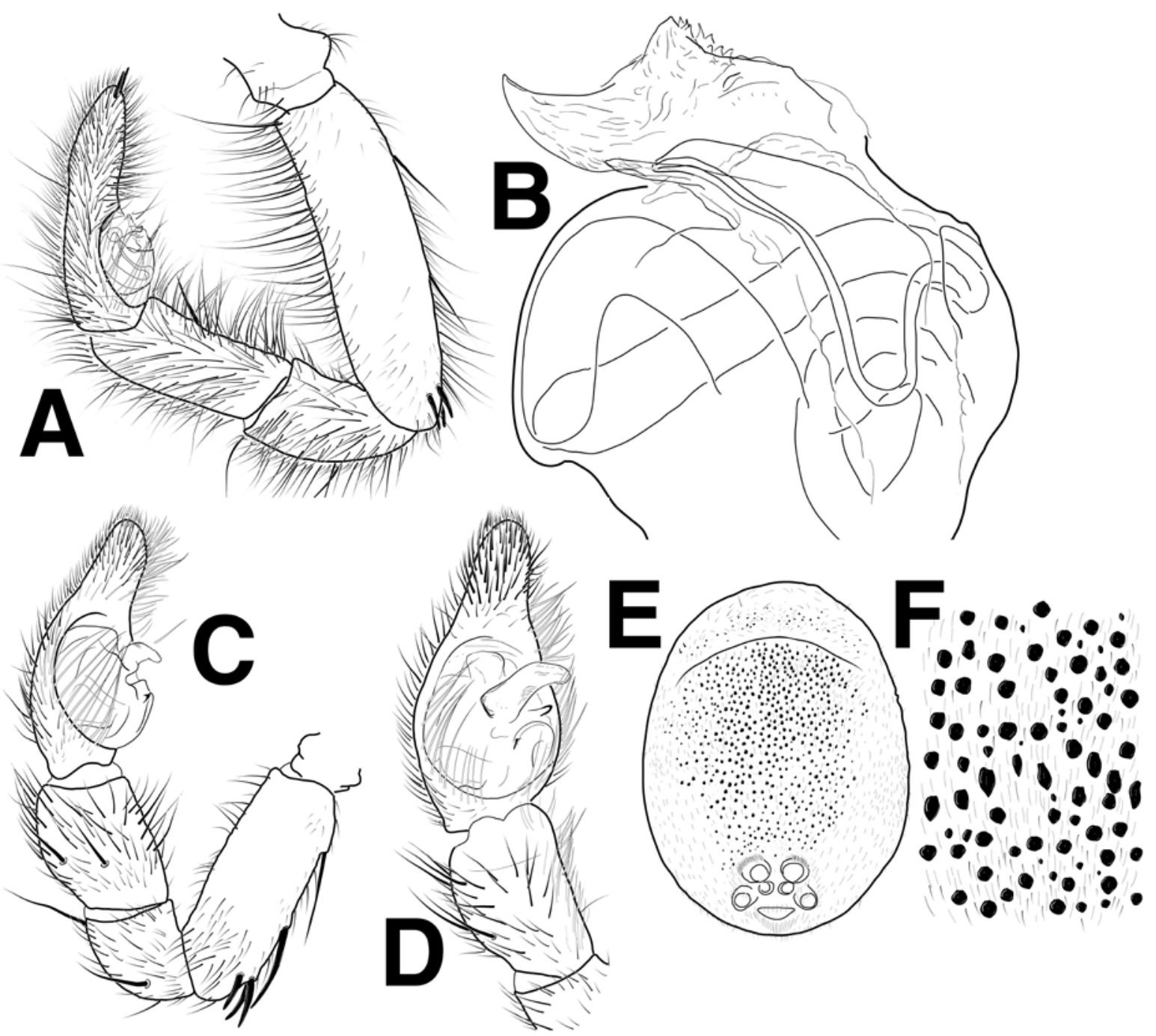

Figure 20. Lycosidae males. A+B Arctosa leopardus pedipalp prolateral (A) and expanded bulb (B). C-F Hygrolycosa rubrofasciata pedipalp lateral (C) and ventral (D), opisthosoma ventral (E) and detail (F). 


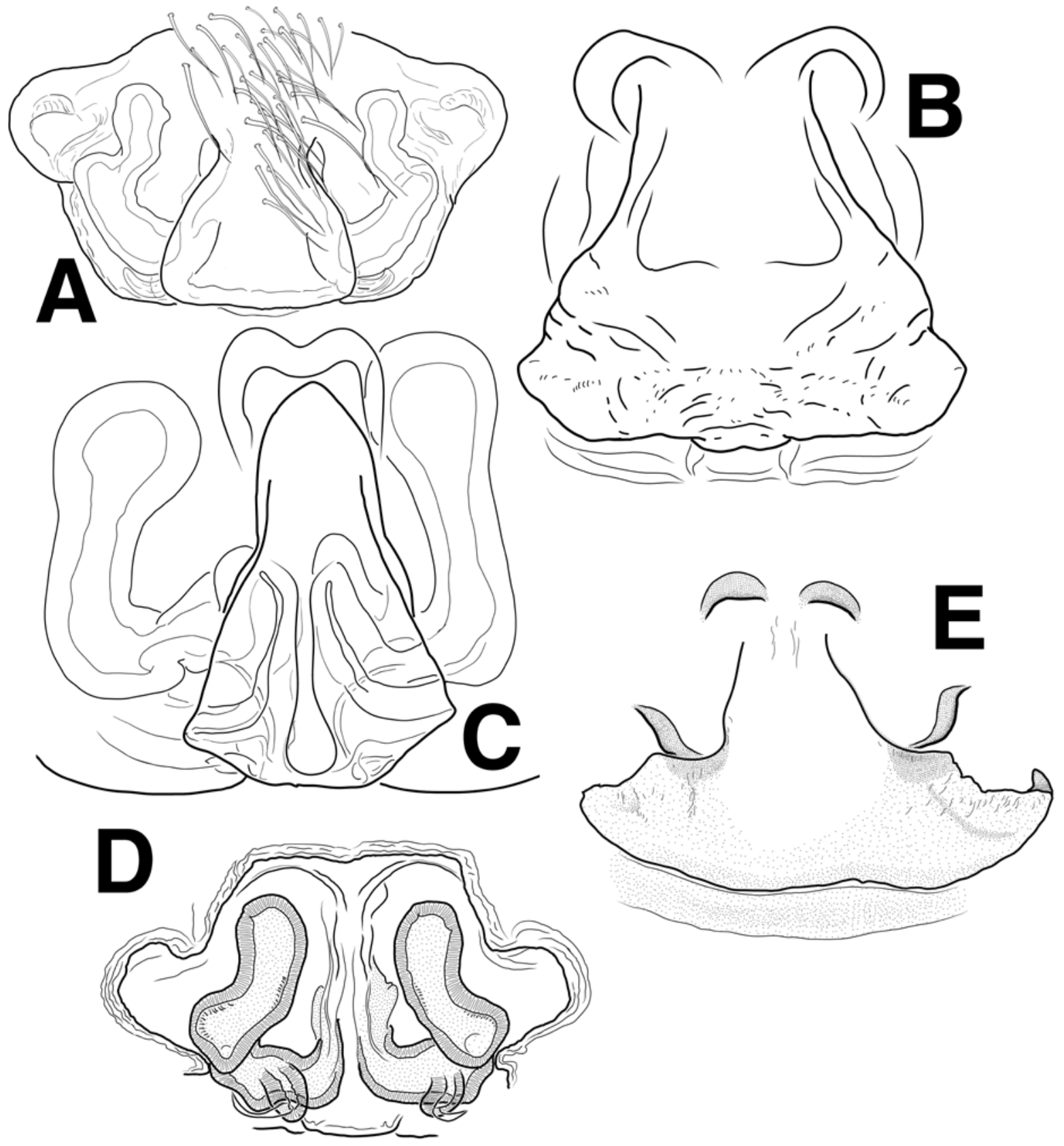

Figure 21. Lycosidae females. A Pardosa femoralis epigynum. B Pardosa mixta epigynum. C Pardosa morosa epigynum. D Pardosa pullata vulva. E Pardosa sp. epigynum. 

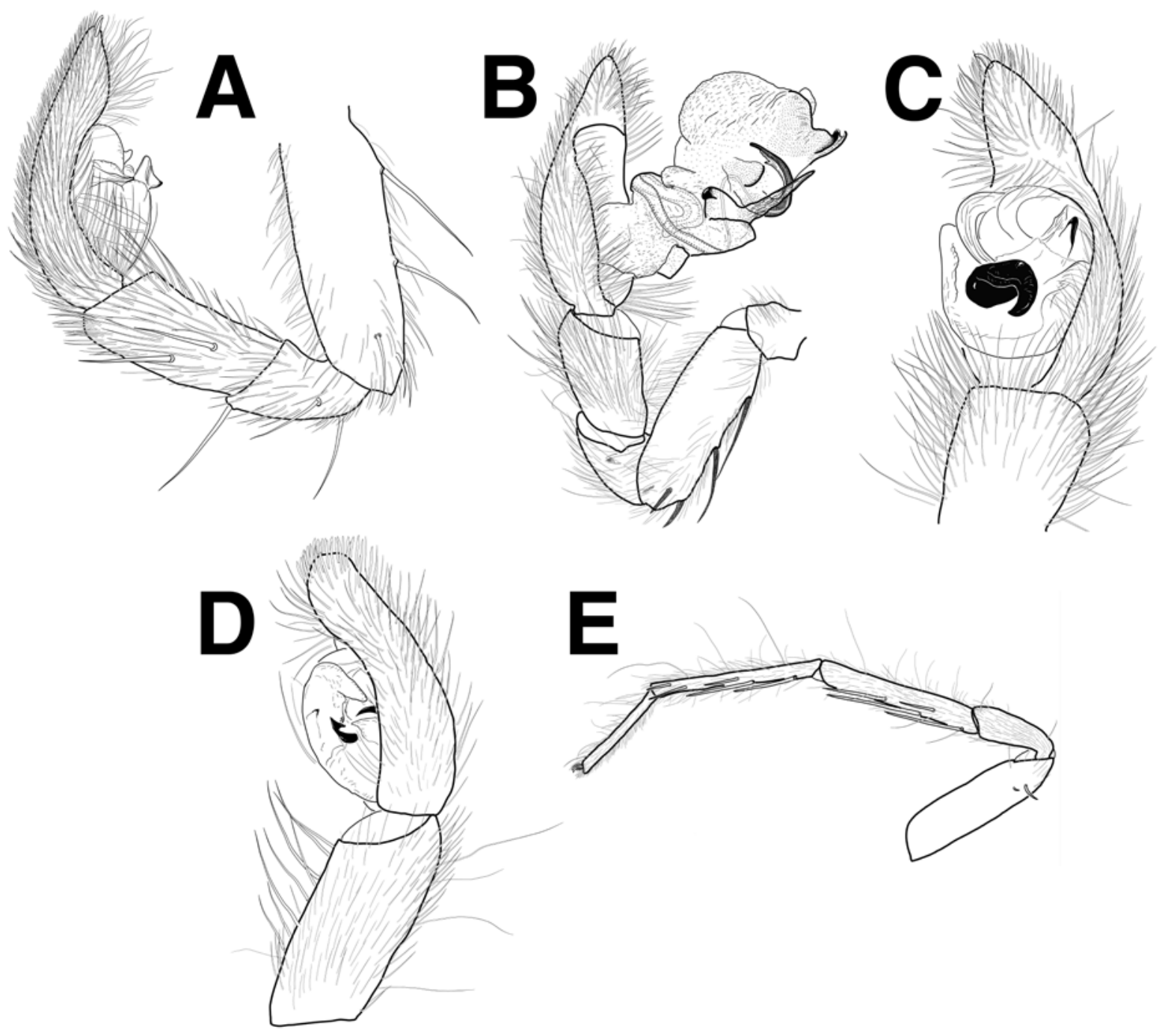

Figure 22. Lycosidae males. A Pardosa morosa pedipalp prolateral. B Pardosa pullata pedipalp expanded. C Pyrenecosa rupicola pedipalp ventral. D+E Trabea cazorla pedipalp retrolateral (D) and leg I prolateral (E). 


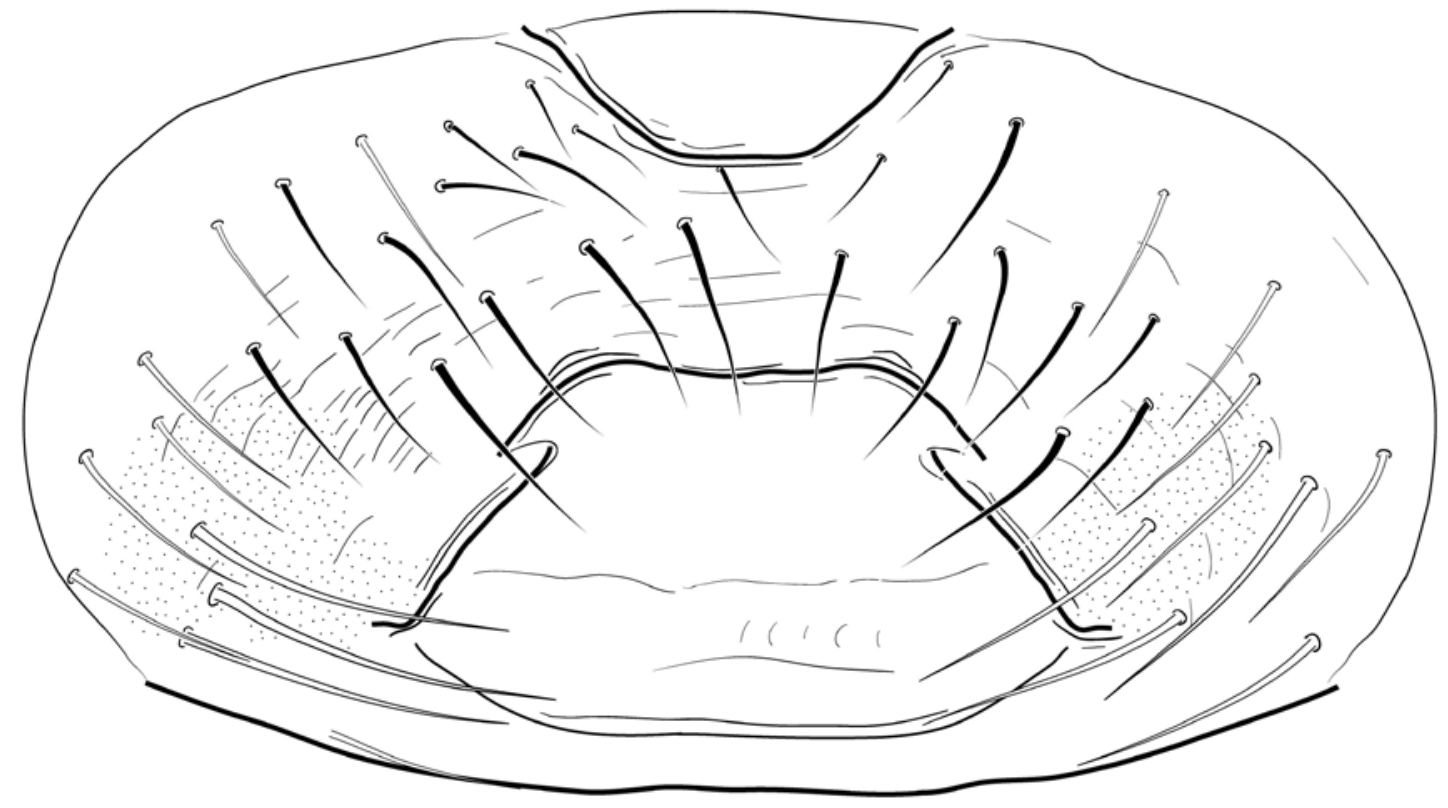

Figure 23. Mimetidae. Mimetus laevigatus epigynum. 

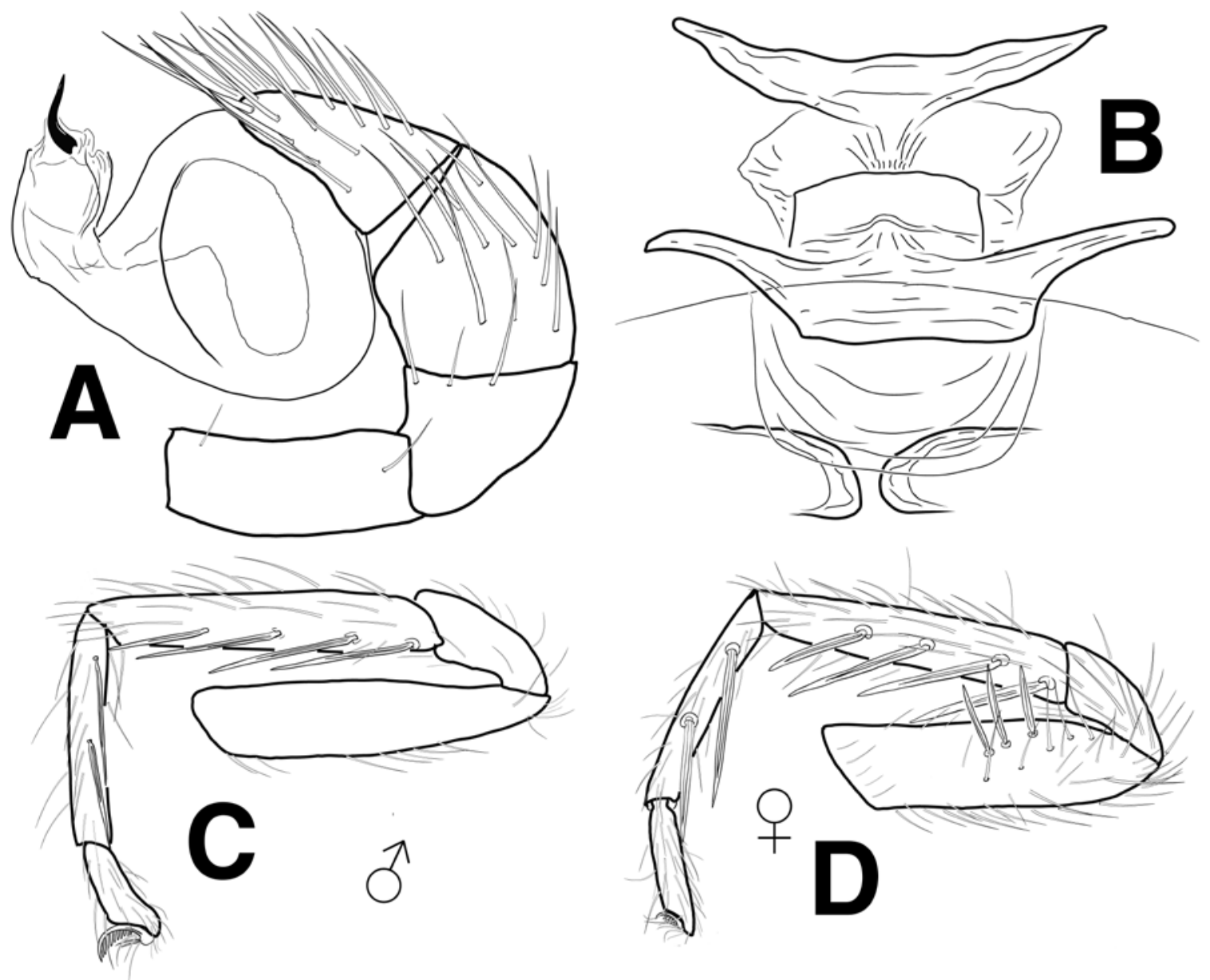

Figure 24. Oonopidae. A-D Oonops procerus pedipalp (A), endogynum dorsal (B), leg I, prolateral, male (C) and female (D).

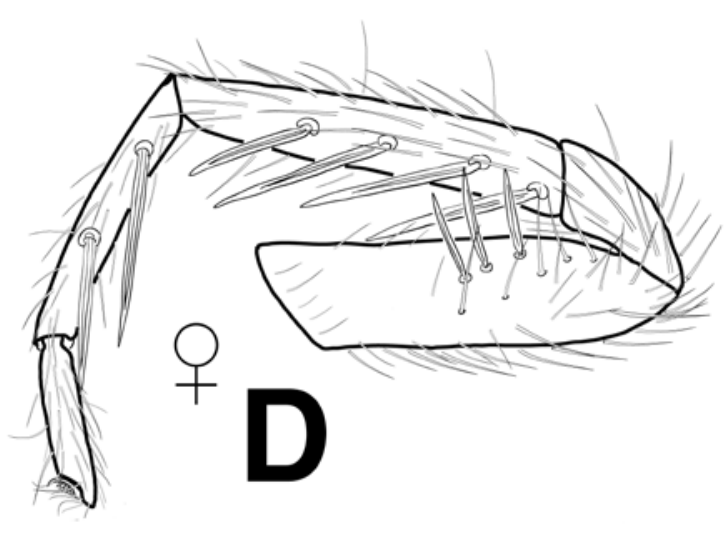



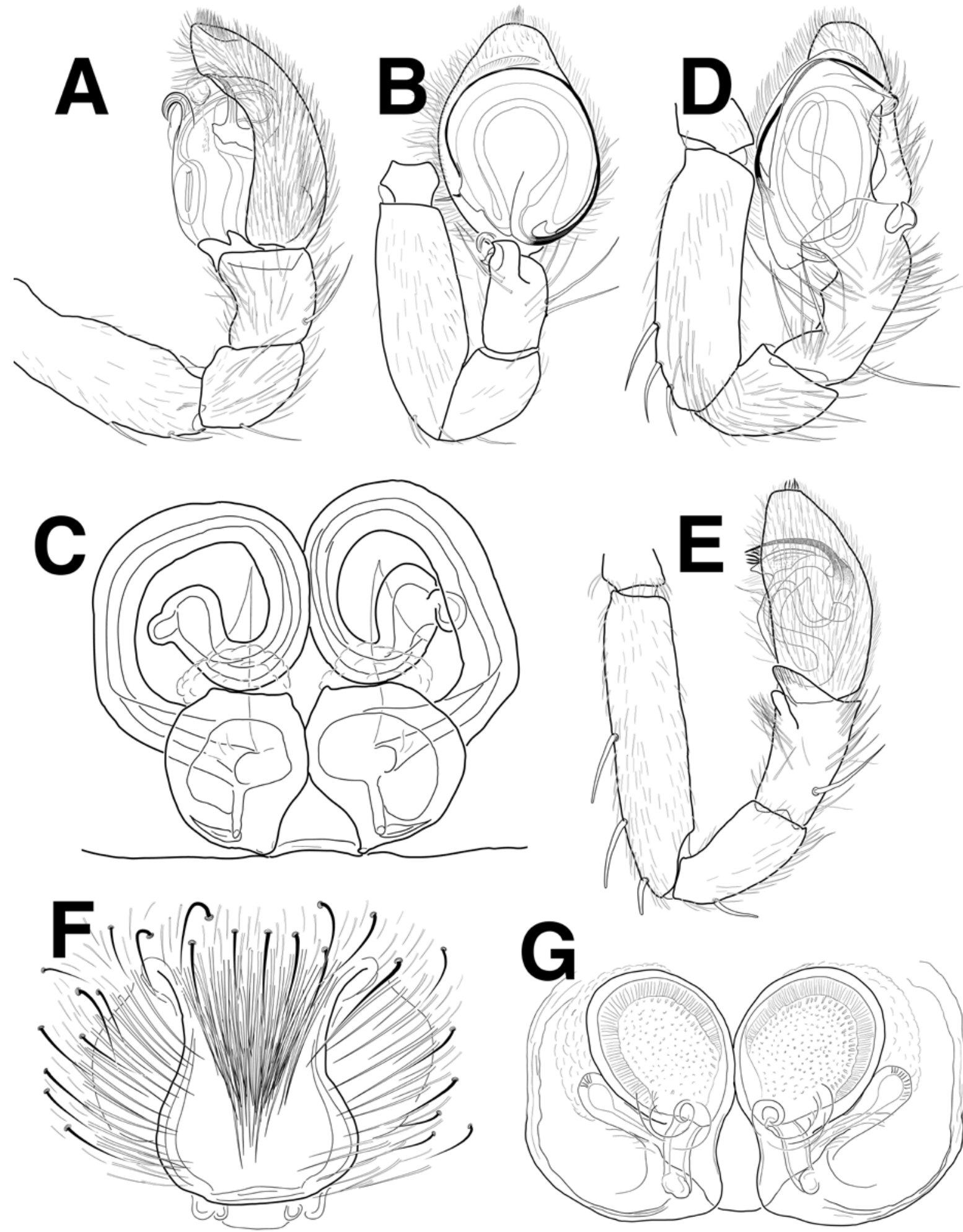

Figure 25. Philodromidae. A Celerrimus duffeyi pedipalp retrolateral. B+C Philodromus dispar pedipalp ventral (B) and vulva (C). D Philodromus emarginatus pedipalp ventral. E Philodromus fuscolimbatus pedipalp dorso-retrolateral. F Thanatus cf. lineatipes epigynum. G Tibellus oblongus vulva. 

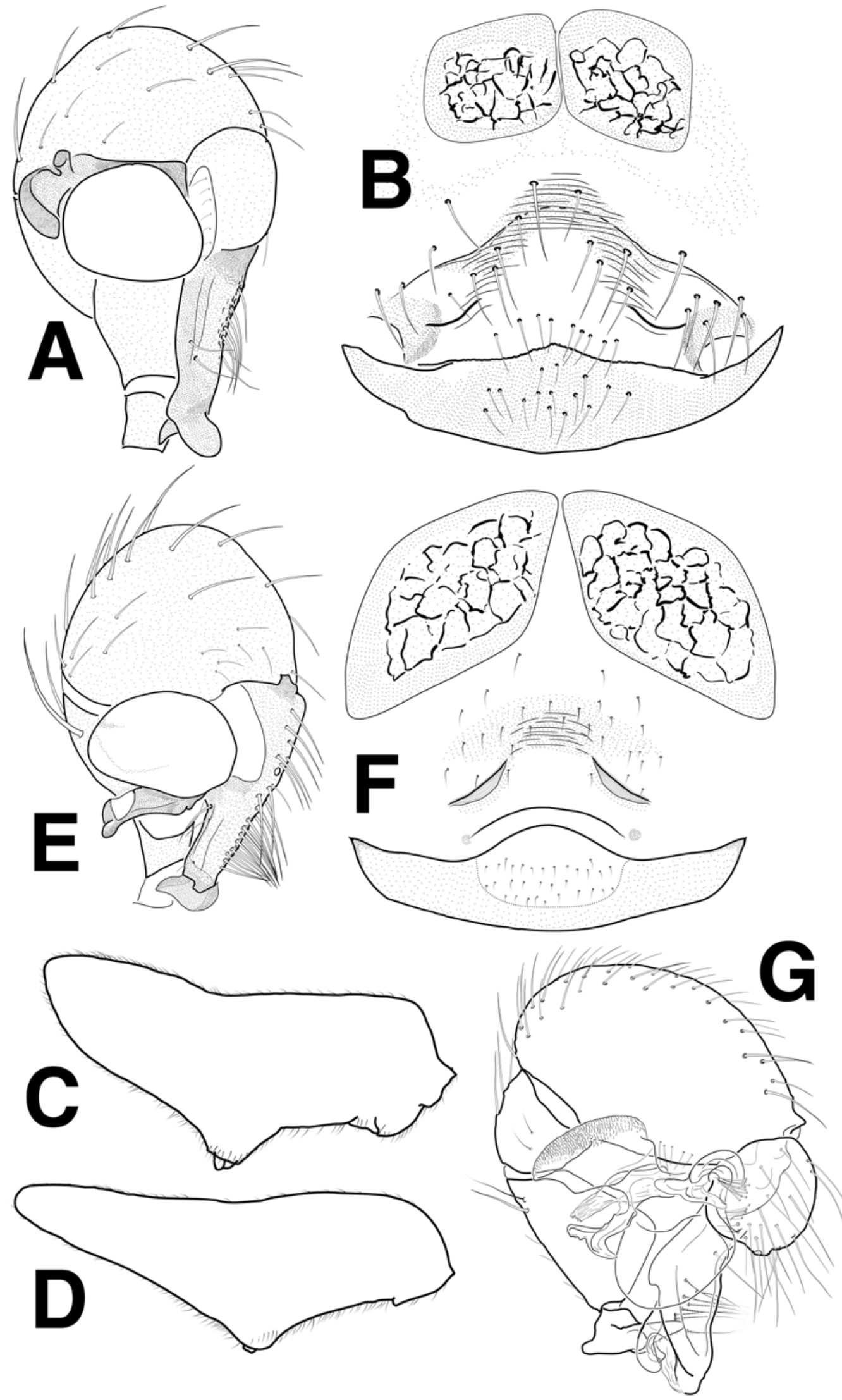

Figure 26. Pholcidae. A-D Holocnemus caudatus pedipalp (A), epigynum (B), and female (C) and male (D) opisthosoma. E, F Holocnemus hispanicus pedipalp (E) and epigynum (F). G Pholcus opilionoides pedipalp. 

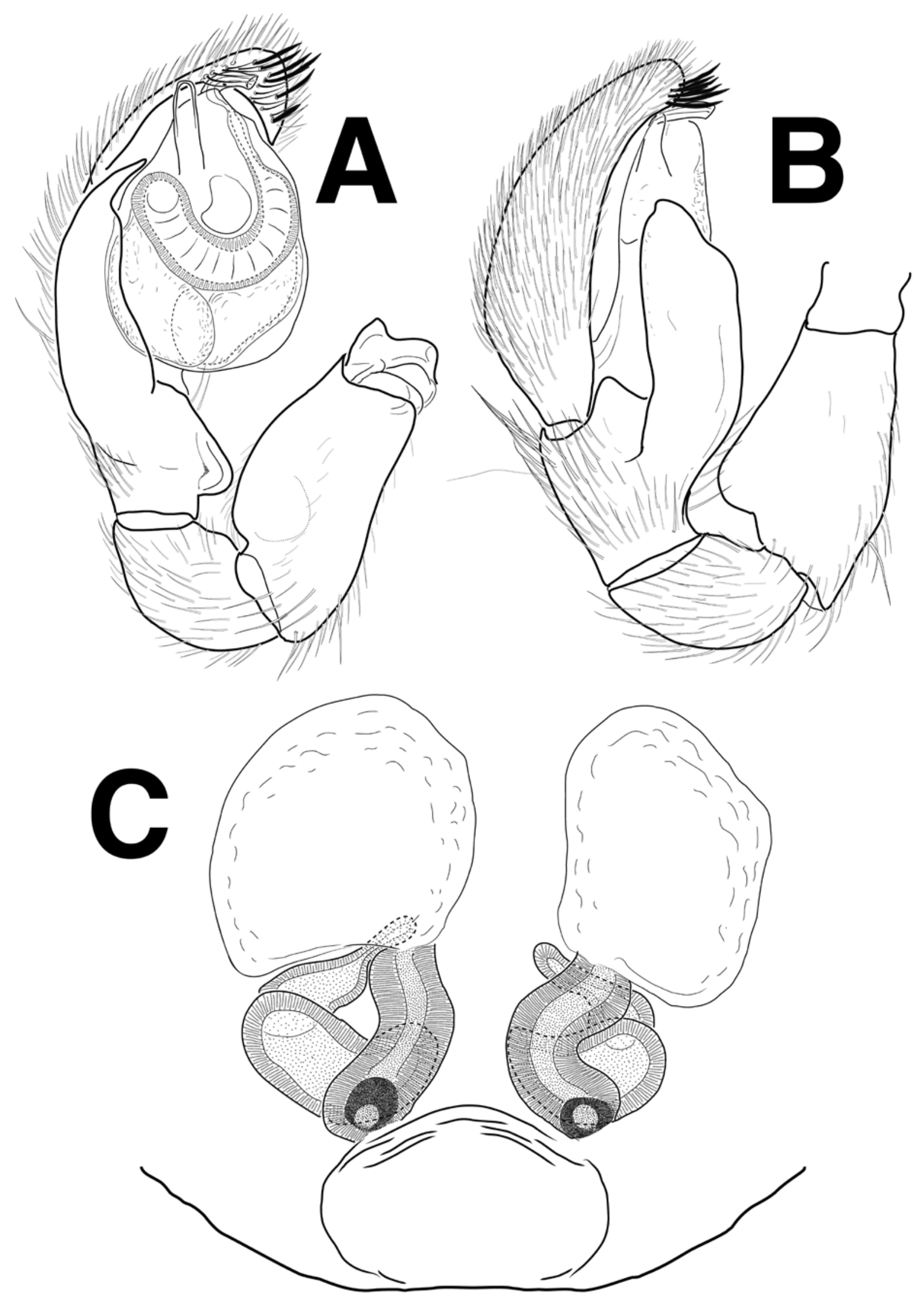

Figure 27. Phrurolithidae. A, B Phrurolithus festivus pedipalp, retrolateral-ventral (A) and retrolateral (B). Phrurolithus cf. szilyi vulva. 


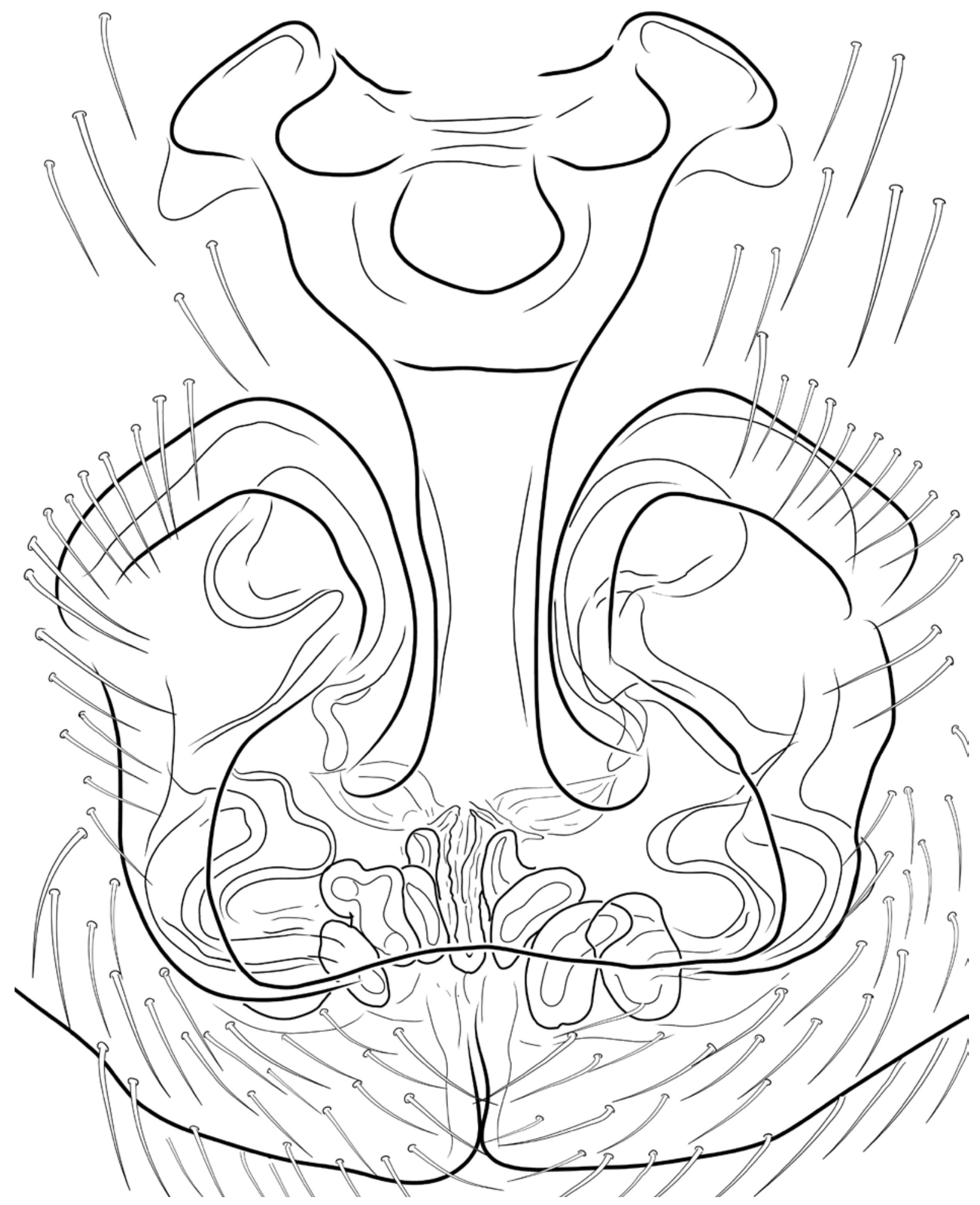

Figure 28. Pisauridae. Pisaura mirabilis epigynum. 

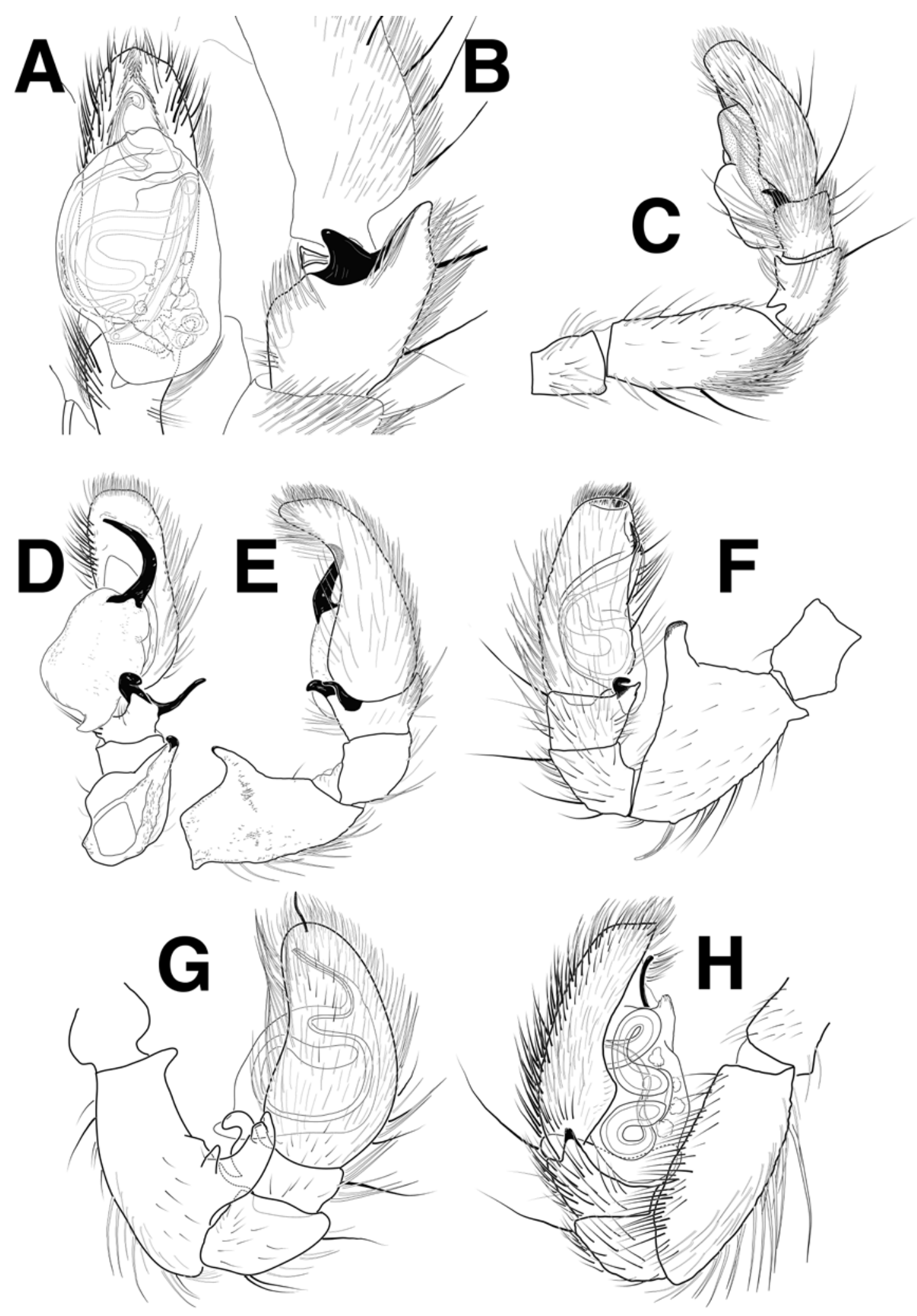

Figure 29. Salticidae males. A, B Aelurillus v-insignitus ventral (A) and retrolateral (B). C Habrocestum cf. ibericum retrolateral. D, E Heliophanus cupreus ventral (D) and retrolateral (E). F Heliophanus patagiatus dorsolateral. G Heliophanus tribulosus dorsolateral. H Iberattus semiglabratus retrolateral. 


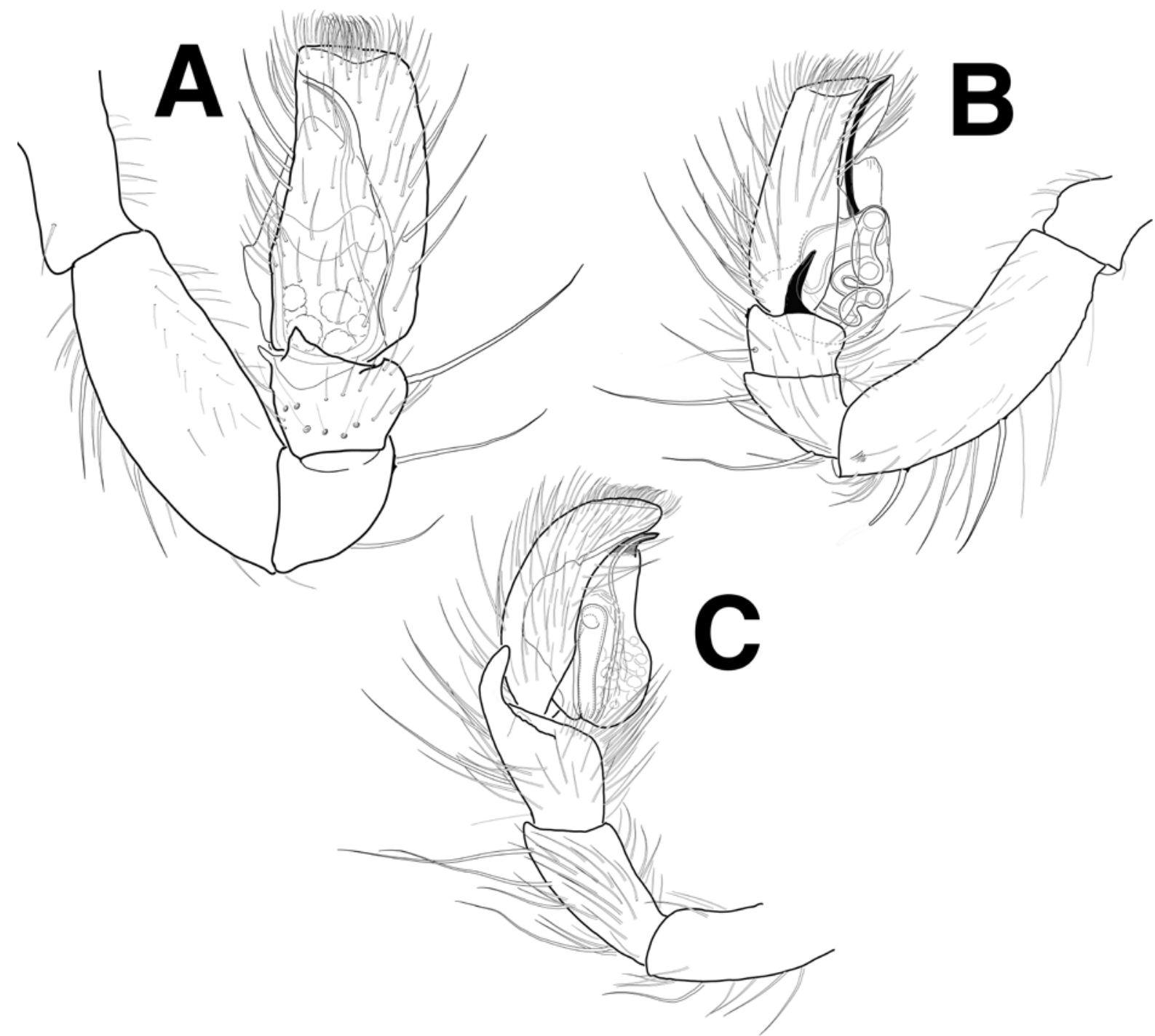

Figure 30. Salticidae males. A Icius hamatus dorsolateral. B Macaroeris nidicolens retrolateral. C Salticus confusus retrolateral. 

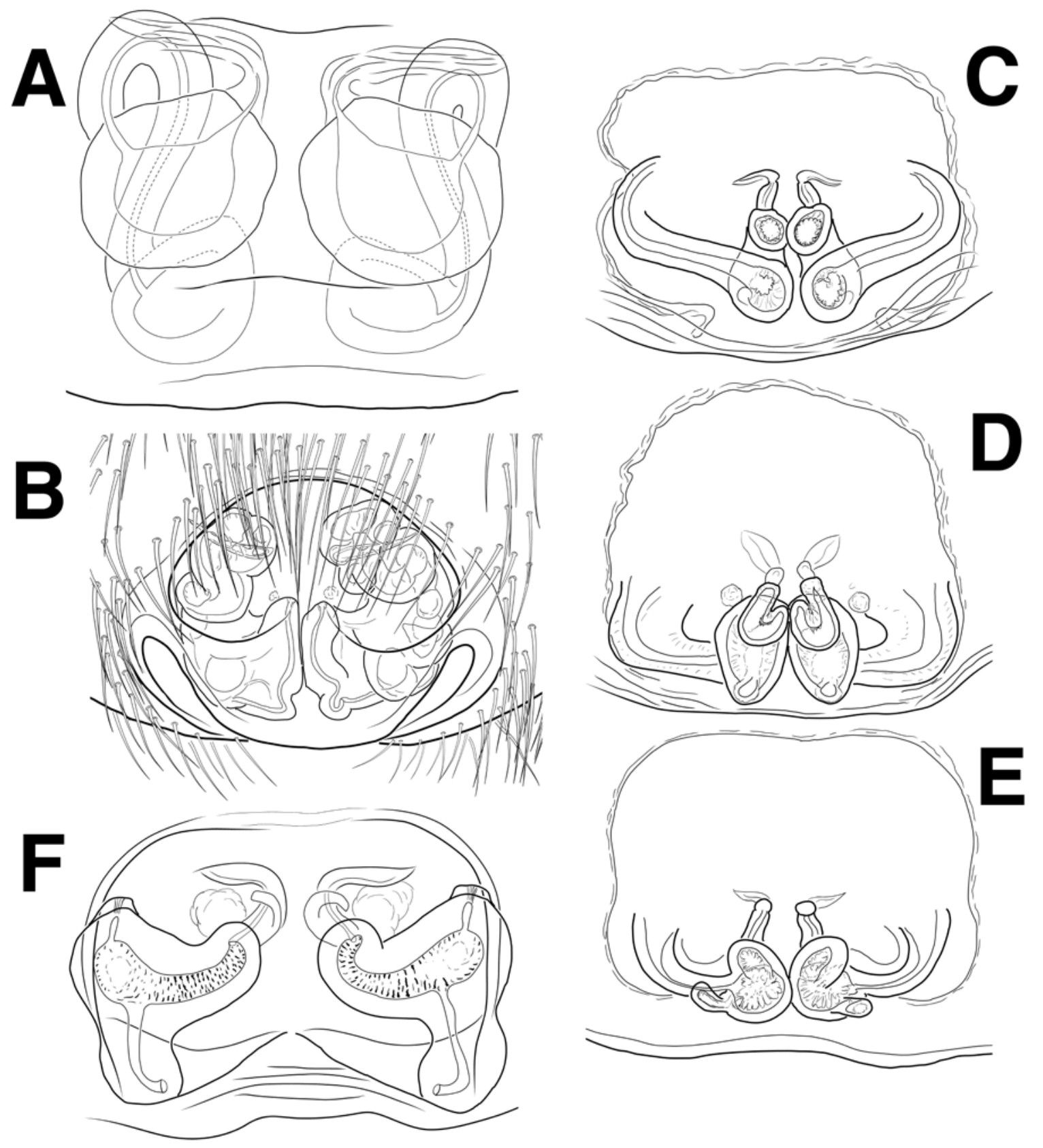

Figure 31. Salticidae females. A Carrhotus xanthogramma vulva. B Evarcha falcata epigynum. C-D Heliophanus cupreus vulva variations. F Heliophanus tribulosus vulva. 

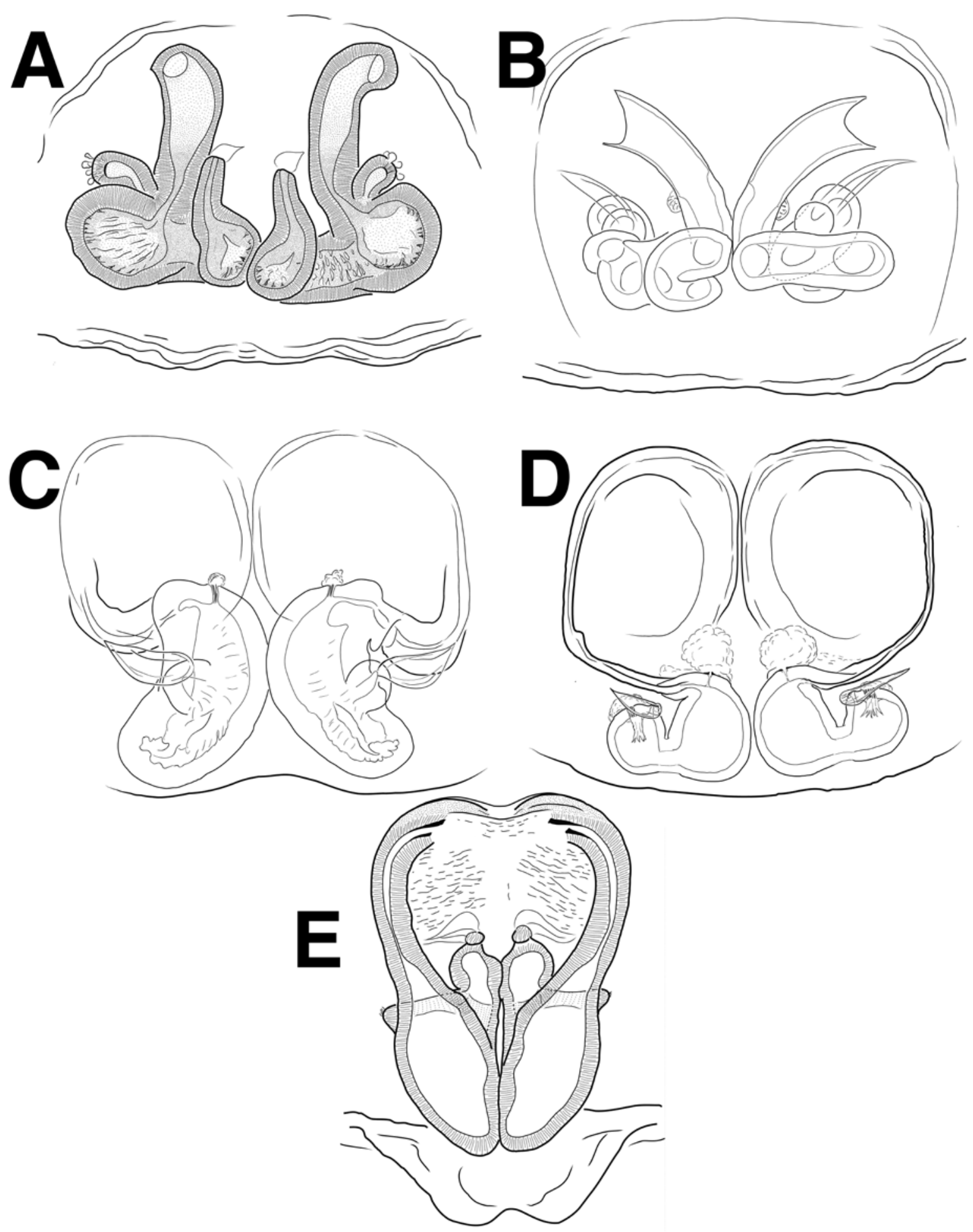

Figure 32. Salticidae female vulvae. A Icius hamatus. B Macaroeris nidicolens. C Pseudeuophrys erratica. D Pseudeuophrys lanigera. E Salticus confusus. 


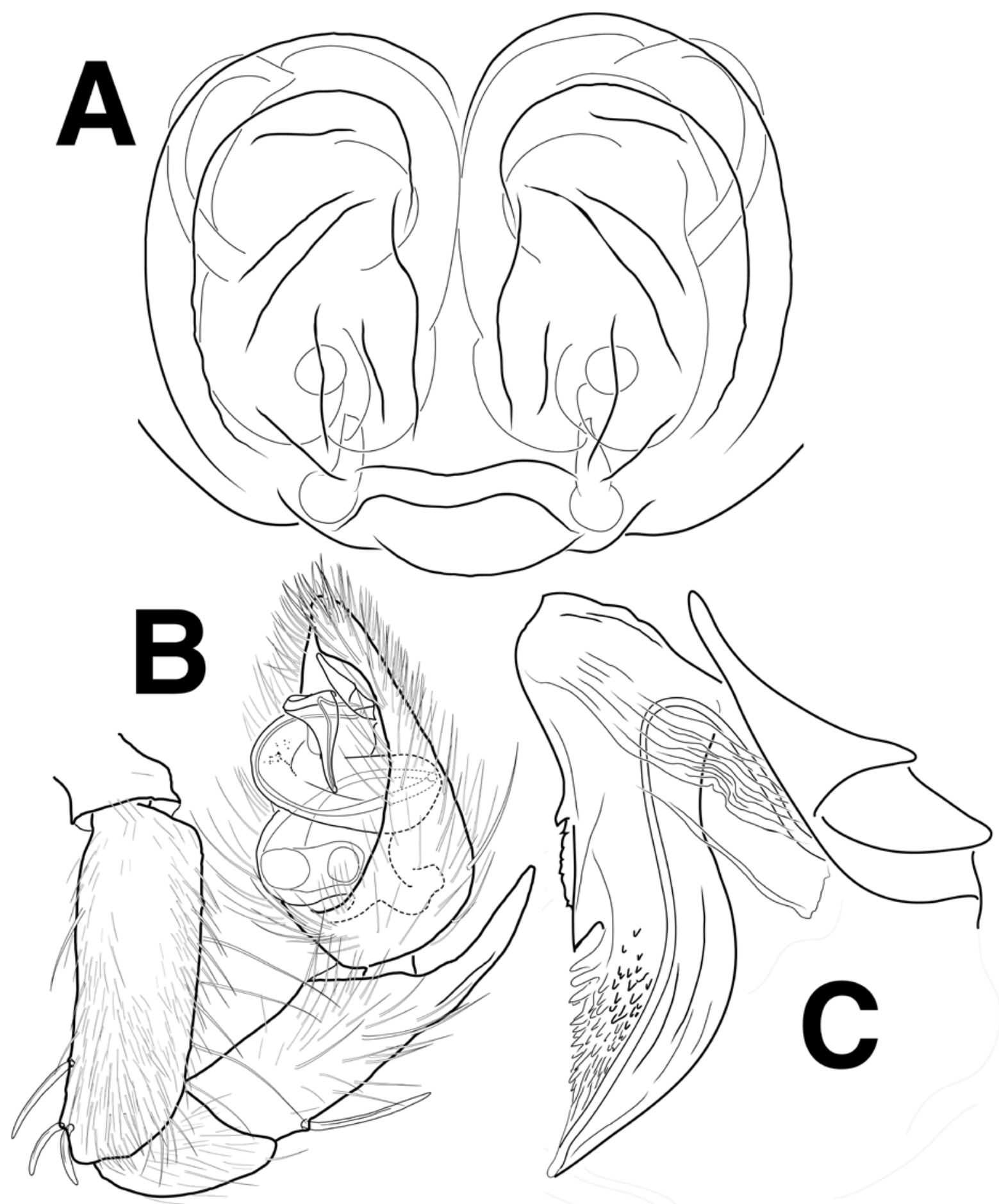

Figure 33. Sparassidae. A Micrommata formosa epigynum. B, C Micrommata virescens pedipalp (B) and embolus (C). 


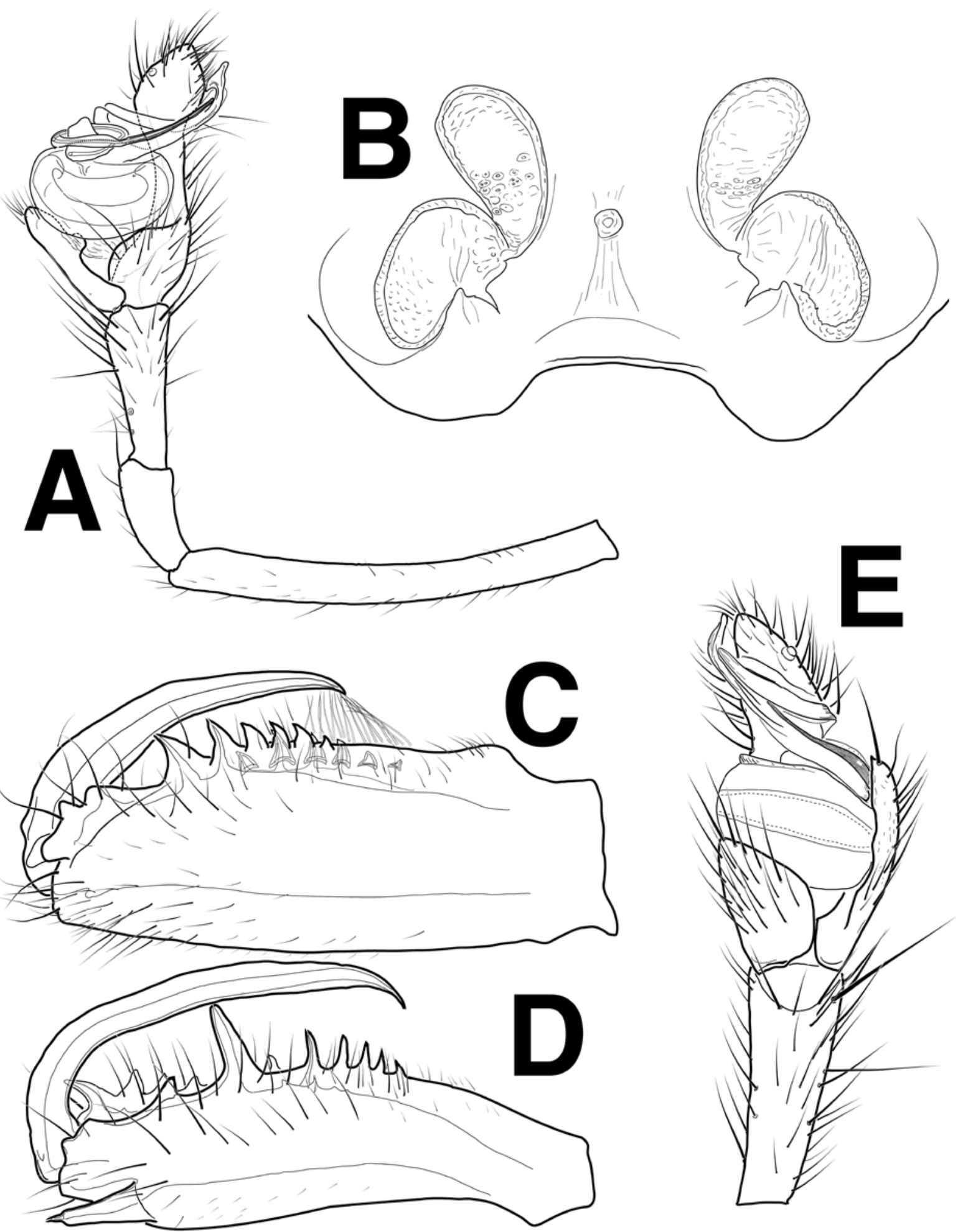

Figure 34. Tetragnathidae. A-D Tetragnatha extensa pedipalp (A), vulva (B), and female (C) and male (D) chelicerae. E Tetragnatha intermedia pedipalp. 

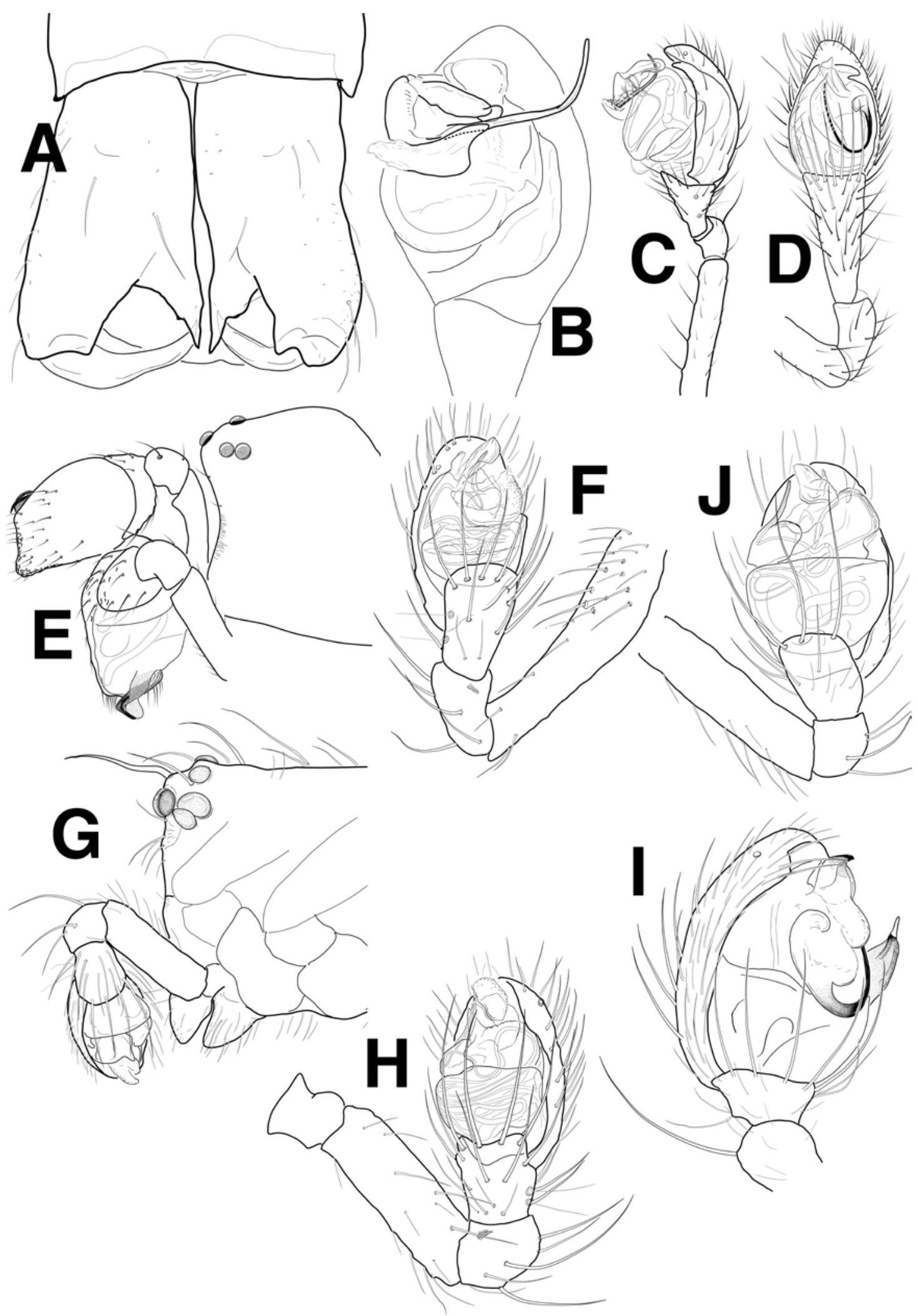

Figure 35. Theridiidae males. A-C Enoplognatha afrodite chelicerae (A), pedipalp ventral (B) and lateral (C), slightly expanded. D Enoplognatha franzi pedipalp ventral. E Euryopis margaritatus pedipalps and prosoma, lateral. F Phylloneta sisyphia pedipalp ventral. $\mathbf{G}+\mathbf{H}$ Theridion betteni prosoma (G) and pedipalp, ventral (H). I Theridion hannoniae pedipalp prolateral. J Theridion mystaceum pedipalp ventral. 

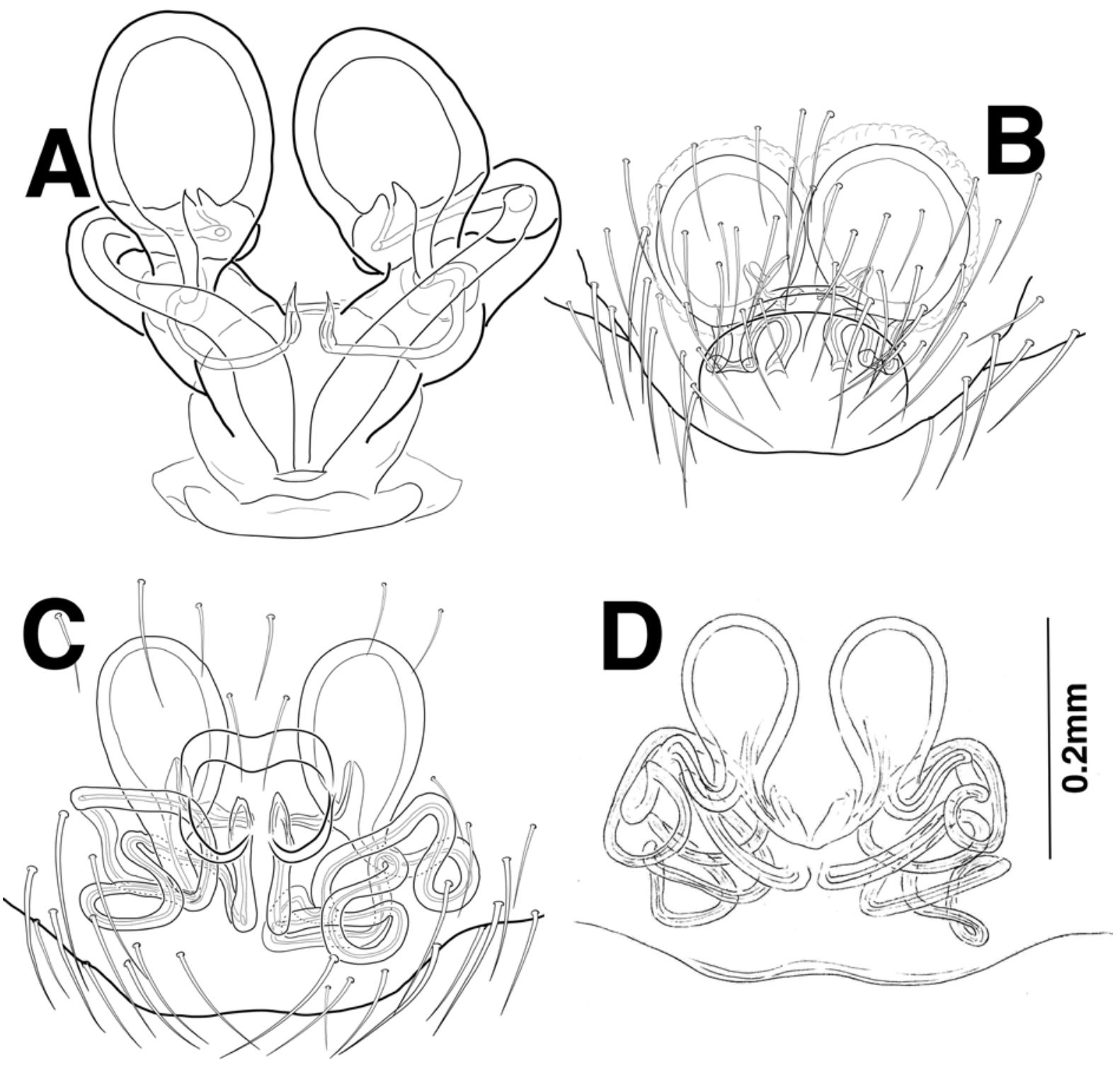

Figure 36. Theridiidae females. A Robertus mediterraneus vulva. B Theridion betteni epigyne. $\mathbf{C}+\mathbf{D}$ Theridion wiehlei epigyne (C) and vulva of a specimen from Algeria in Simon's collection in the Muséum national d'Histoire naturelle, Paris (D, courtesy of Barbara Knoflach-Thaler). 


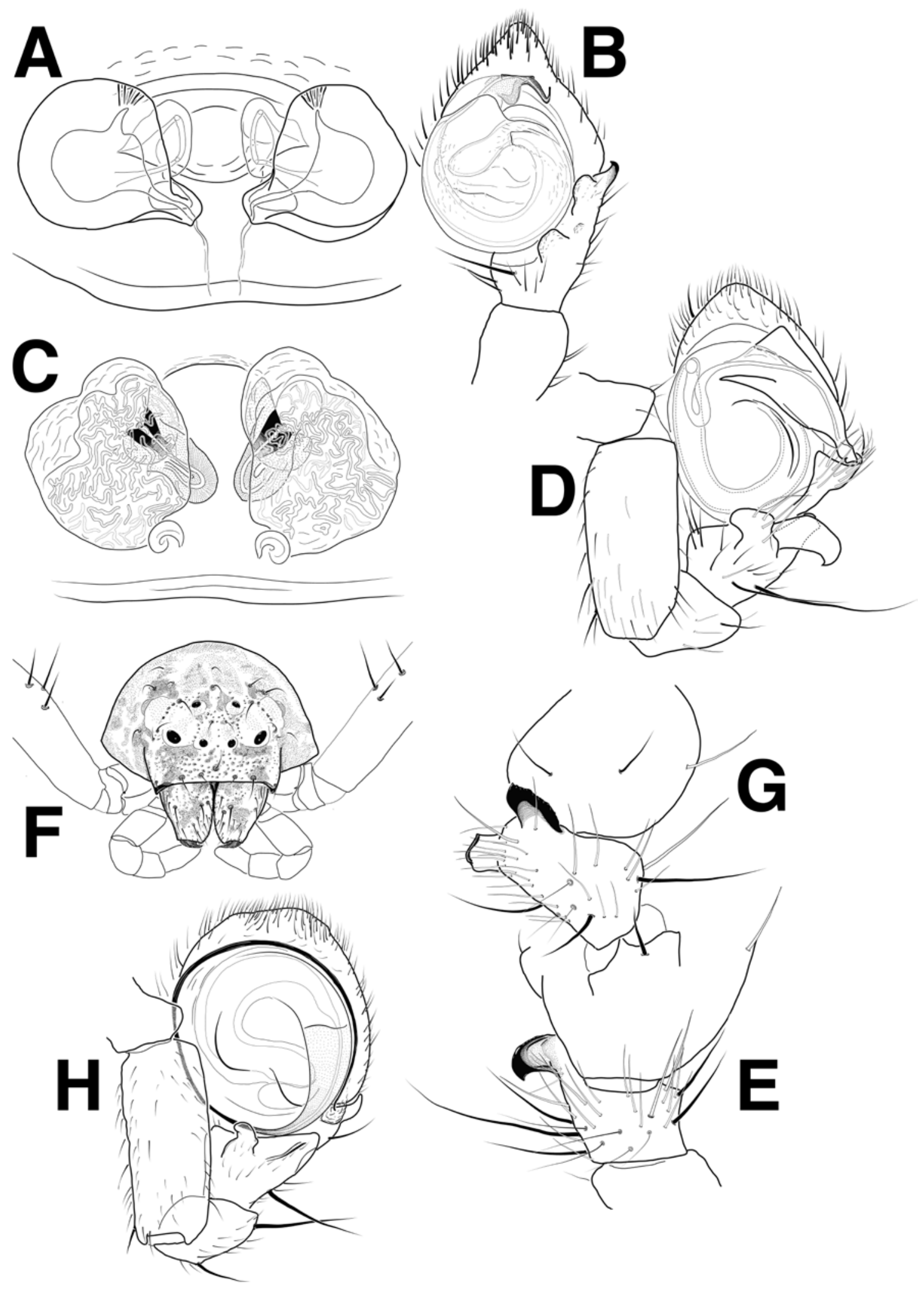

Figure 37. Thomisidae. A+B Misumena vatia vulva (A) and pedipalp ventral (B). C Thomisus onustus vulva. D+E Tmarus punctatissimus pedipalp ventral (D) and tibial apophysis dorsal (E). F+G Tmarus stellio female face (F) and male tibial apophysis dorsal (G). H Xysticus kempeleni pedipalp ventral. 


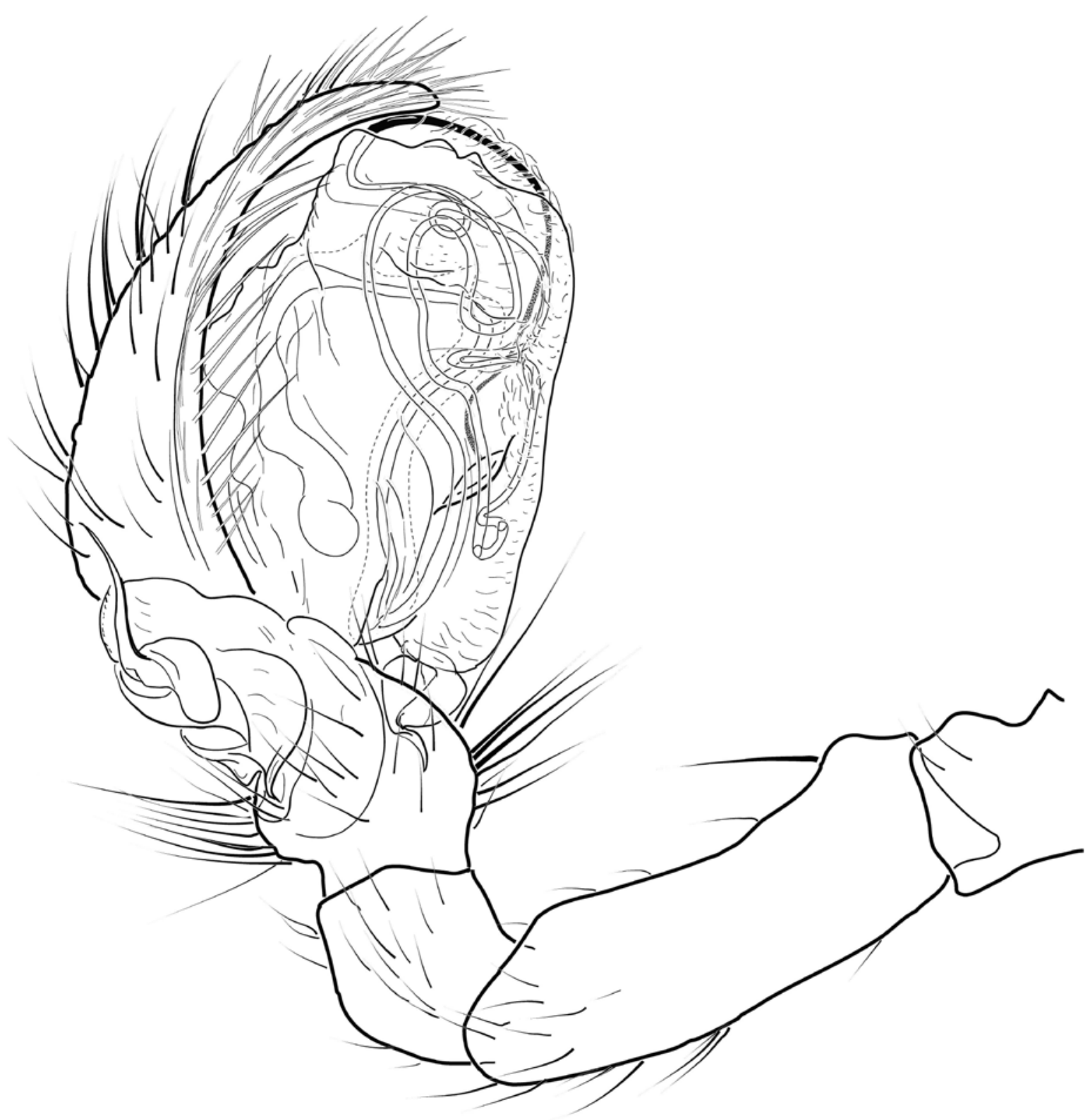

Figure 38. Titanoecidae. Titanoeca monticola pedipalp, retrolateral. 


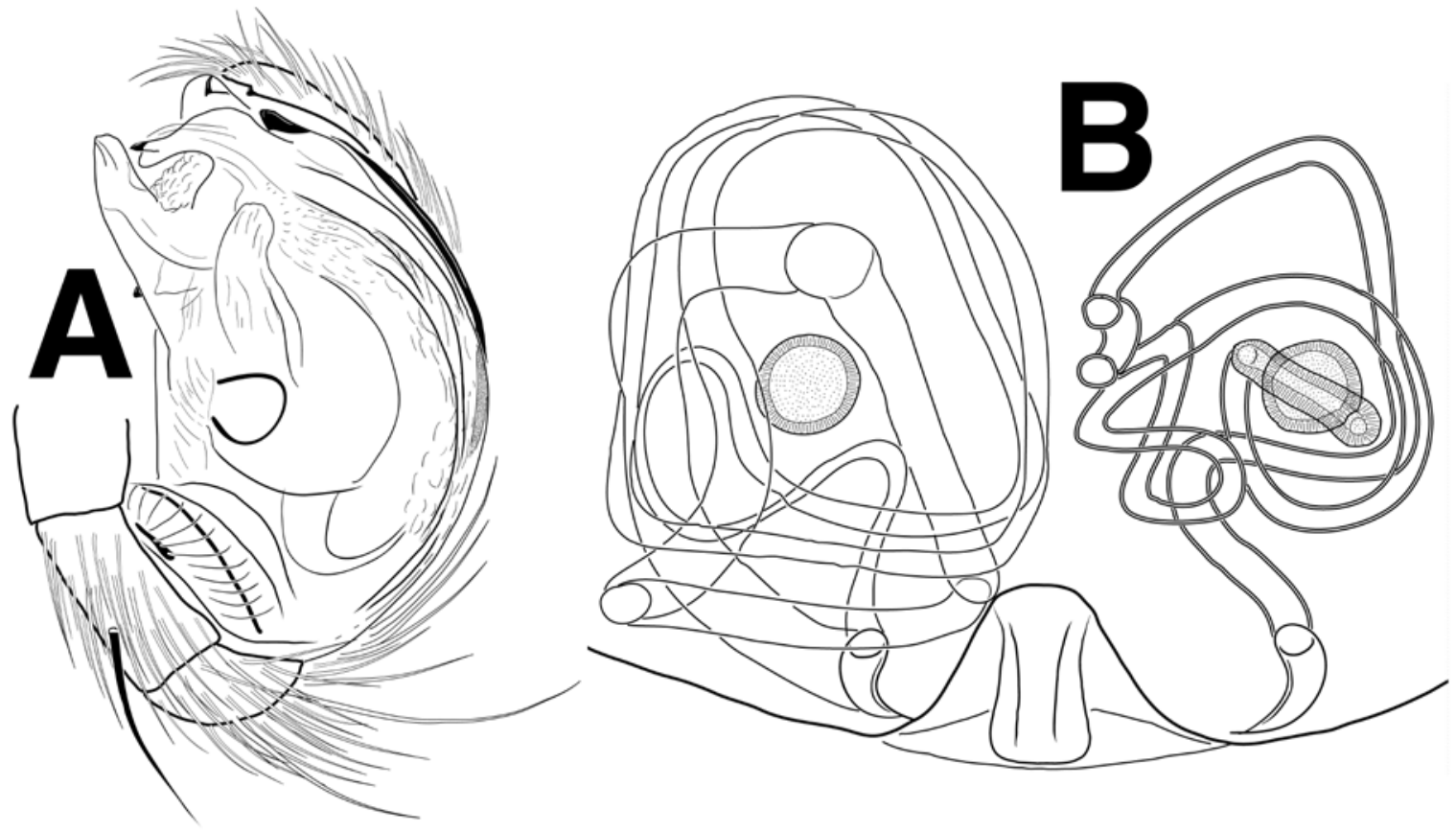

Figure 39. Uloboridae. Hyptiotes flavidus pedipalp, prolateral (A) and epigynum (B).
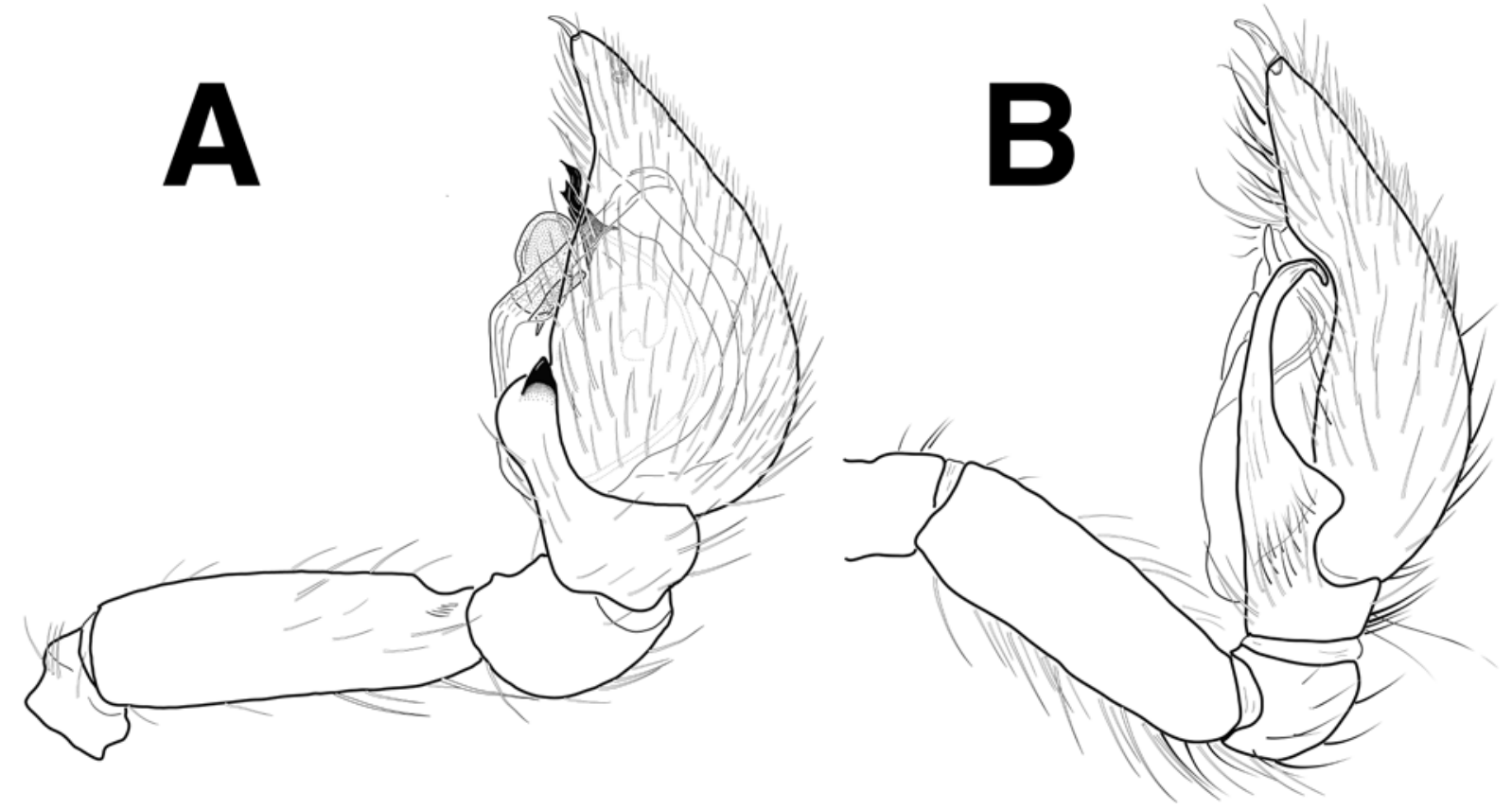

Figure 40. Zodariidae male pedipalps retrolateral. A Zodarion fuscum. B Zodarion rudyi. 